\title{
Spatially isotropic homogeneous spacetimes
}

\author{
José Figueroa-O'Farrill $^{a}$ and Stefan Prohazka ${ }^{b}$ \\ ${ }^{a}$ Maxwell Institute and School of Mathematics, The University of Edinburgh, \\ James Clerk Maxwell Building, Peter Guthrie Tait Road, Edinburgh EH9 3FD, Scotland, U.K. \\ ${ }^{b}$ Université Libre de Bruxelles and International Solvay Institutes, \\ Physique Mathématique des Interactions Fondamentales, \\ Campus Plaine - CP 231, B-1050 Bruxelles, Belgium \\ E-mail: j.m.figueroa@ed.ac.uk, stefan.prohazka@ulb.be.ac
}

ABSTRACT: We classify simply-connected homogeneous $(D+1)$-dimensional spacetimes for kinematical and aristotelian Lie groups with $D$-dimensional space isotropy for all $D \geq 0$. Besides well-known spacetimes like Minkowski and (anti) de Sitter we find several new classes of geometries, some of which exist only for $D=1,2$. These geometries share the same amount of symmetry (spatial rotations, boosts and spatio-temporal translations) as the maximally symmetric spacetimes, but unlike them they do not necessarily admit an invariant metric. We determine the possible limits between the spacetimes and interpret them in terms of contractions of the corresponding transitive Lie algebras. We investigate geometrical properties of the spacetimes such as whether they are reductive or symmetric as well as the existence of invariant structures (riemannian, lorentzian, galilean, carrollian, aristotelian) and, when appropriate, discuss the torsion and curvature of the canonical invariant connection as a means of characterising the different spacetimes.

Keywords: Space-Time Symmetries, Differential and Algebraic Geometry

ARXIV EPRINT: 1809.01224

In memoriam Andrew Ranicki 


\section{Contents}

1 Introduction 1

1.1 Motivation and contextualisation 1

1.2 Overview of results 4

1.3 Organisation of the paper 8

$\begin{array}{ll}1.4 \text { Reader's guide } & 10\end{array}$

2 Kinematical spacetimes $\quad 11$

$\begin{array}{lll}2.1 \text { Basic definitions } & 11\end{array}$

$\begin{array}{lll}2.2 & \text { Summary of main results } & 14\end{array}$

3 Classification of kinematical Lie pairs $\quad 16$

$\begin{array}{lll}3.1 & \text { Lie pairs for } D \geq 3 & 17\end{array}$

3.1.1 Lie pairs associated to Lie algebra \#1 18

$\begin{array}{lll}3.1 .2 & \text { Lie pairs associated to Lie algebra \#2 } & 19\end{array}$

$\begin{array}{lll}3.1 .3 & \text { Lie pairs associated to Lie algebra } \# 3_{\gamma} & 19\end{array}$

3.1.4 Lie pairs associated to Lie algebra \#4 20

3.1.5 Lie pairs associated to Lie algebra \#5 20

3.1.6 Lie pairs associated to Lie algebra \#6 20

3.1.7 Lie pairs associated to Lie algebra $\# 7 \chi \quad 21$

3.1.8 Lie pairs associated to Lie algebra \#8 21

3.1.9 Lie pairs associated to Lie algebra \#9 21

3.1 .10 Lie pairs associated to Lie algebra \#10 $\_22$

3.1.11 Lie pairs associated to Lie algebra \#11 22

3.1.12 Lie pairs associated to Lie algebra \#12 $\varepsilon$

3.1.13 Summary 23

$\begin{array}{lll}3.2 & \text { Lie pairs unique to } D=3 & 23\end{array}$

3.2.1 Lie pairs associated to Lie algebra \#13 $\varepsilon_{\varepsilon}$

3.2.2 Lie pairs associated to Lie algebra \#14 24

3.2.3 Lie pairs associated to Lie algebra \#15 24

3.2.4 Lie pairs associated to Lie algebra \#16 24

$\begin{array}{ll}3.2 .5 & \text { Lie pairs associated to Lie algebra \#17 } 25\end{array}$

3.2.6 Lie pairs associated to Lie algebra \#18 25

$\begin{array}{lll}3.2 .7 & \text { Summary } & 25\end{array}$

$\begin{array}{lll}3.3 & \text { Lie pairs for } D=2 & 25\end{array}$

3.3.1 Lie pairs associated to Lie algebra \#19 27

3.3.2 Lie pairs associated to the Lie algebra \#20 27

3.3.3 Lie pairs associated to Lie algebra \#21 27

3.3.4 Lie pairs associated to Lie algebras \#22 $2_{\gamma+i \chi}$ and \#23 27

3.3.5 Lie pairs associated to Lie algebra \#24 29

$\begin{array}{lll}3.3 .6 & \text { Lie pairs associated to Lie algebra \#25 } & 29\end{array}$ 
3.3.7 Lie pairs associated to Lie algebra \#26 $\boldsymbol{\varepsilon}_{\varepsilon} \quad 29$

3.3.8 Lie pairs associated to Lie algebra \#27 30

3.3.9 Lie pairs associated to Lie algebra \#28 $8_{\varepsilon} \quad 30$

3.3.10 No Lie pairs associated to Lie algebras \#29 and \#30 30

3.3.11 Lie pairs associated to Lie algebras \#31, \#32 and \#33 $\varepsilon_{\varepsilon} 31$

$\begin{array}{ll}3.3 .12 \text { Summary } & 31\end{array}$

3.4 Lie pairs for $D=1 \quad 31$

3.4.1 Bianchi I 33

3.4.2 Bianchi II 33

3.4.3 Bianchi IV 33

$\begin{array}{lll}3.4 .4 & \text { Bianchi V } & 34\end{array}$

3.4.5 Bianchi $\mathrm{VI}_{0} \quad 34$

3.4.6 Bianchi $\mathrm{VI}_{\chi>0} \quad 35$

$\begin{array}{lll}3.4 .7 & \text { Bianchi } \mathrm{VII}_{0} & 35\end{array}$

3.4.8 Bianchi VII $>>0 \quad 36$

3.4.9 Bianchi VIII 36

3.4.10 Bianchi IX $\quad 36$

$\begin{array}{ll}3.4 .11 \text { Summary } & 37\end{array}$

4 Classification of simply-connected homogeneous spacetimes 37

4.1 Effective Lie pairs 38

4.1.1 Effective Lie pairs for all $D \geq 3 \quad 38$

4.1.2 No effective Lie pairs unique to $D=3 \quad 38$

4.1.3 Effective Lie pairs for $D=2 \quad 39$

4.1.4 Effective Lie pairs for $D=1 \quad 39$

$\begin{array}{lll}\text { 4.1.5 Summary } & 39\end{array}$

$\begin{array}{lll}4.2 & \text { Geometric realisability } & 39\end{array}$

4.2.1 Riemannian maximally symmetric spaces 41

$\begin{array}{lll}4.2 .2 & \text { A sufficient criterion } & 41\end{array}$

4.2.3 Two-dimensional spacetimes $\quad 41$

4.2.4 The carrollian light cone $\quad 43$

4.2.5 Symmetric carrollian spacetimes 44

5 Limits between homogeneous spacetimes $\quad 47$

$\begin{array}{lll}5.1 & \text { Contractions } & 47\end{array}$

$\begin{array}{lll}5.2 & D \geq 3 & 47\end{array}$

5.2.1 AdSG $_{\chi} \rightarrow \mathrm{G} \quad 50$

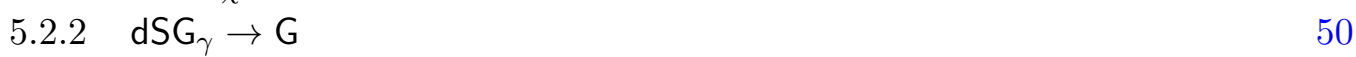

$\begin{array}{lll}5.2 .3 \quad \mathrm{LC} \rightarrow \mathrm{C} & 50\end{array}$

$\begin{array}{lll}5.2 .4 \quad \text { LC } \rightarrow \text { TS } & 50\end{array}$

$\begin{array}{lll}5.2 .5 \quad \mathrm{TS} \rightarrow \mathrm{S} & 51\end{array}$

5.2.6 A non-contracting limit $\quad 51$

$5.3 D=2 \quad 51$ 
$5.4 D=1 \quad 52$

5.4.1 $\mathrm{S} 17 \rightarrow \mathrm{S} 18$ and $\mathrm{S} 17 \rightarrow \mathrm{S} 7 / \mathrm{S} 13 \quad 53$

$\begin{array}{lll}5.4 .2 & \mathrm{~S} 19_{\chi} \rightarrow \mathrm{S} 7 / \mathrm{S} 13 & 54\end{array}$

$5.4 .3 \mathrm{~S} 20_{\chi} \rightarrow \mathrm{S} 7 / \mathrm{S} 13 \quad 54$

5.4.4 Additional limits $\quad 55$

6 Some geometrical properties of homogeneous spacetimes $\quad 55$

$\begin{array}{lll}6.1 \text { Basic notions } & 55\end{array}$

6.1.1 Reductive and symmetric Lie pairs 55

$\begin{array}{ll}\text { 6.1.2 The linear isotropy representation } & 55\end{array}$

$\begin{array}{lll}6.1 .3 & \text { Invariant structures } & 56\end{array}$

$\begin{array}{ll}\text { 6.1.4 Parity and time reversal } & 57\end{array}$

6.2 Invariant connections, curvature and torsion 58

6.2.1 Invariant connections for spacetime LC $\quad 60$

6.3 Summary of properties of homogeneous spacetimes 61

$\begin{array}{lll}\text { 6.3.1 Flat symmetric spacetimes } & 61\end{array}$

$\begin{array}{lll}\text { 6.3.2 } & \text { Non-flat symmetric spacetimes } & 62\end{array}$

$\begin{array}{lll}\text { 6.3.3 Reductive torsional spacetimes } & 63\end{array}$

$\begin{array}{lll}\text { 6.3.4 Summary } & 64\end{array}$

$\begin{array}{lll}7 & \text { Conclusions } & 65\end{array}$

$\begin{array}{ll}\text { A Classification of aristotelian Lie algebras } & 67\end{array}$

$\begin{array}{lr}\text { B Infinitesimal description of homogeneous spaces } & 69\end{array}$

$\begin{array}{lll}\text { B.1 Transitive actions of Lie groups } & 69\end{array}$

$\begin{array}{lll}\text { B.2 Lie pairs } & 70\end{array}$

$\begin{array}{lll}\text { B.3 Geometric realisations } & 72\end{array}$

$\begin{array}{lll}\text { B.4 Simply-connected homogeneous spaces } & 72\end{array}$

\section{Introduction}

\subsection{Motivation and contextualisation}

The laws of physics are to a good approximation invariant under spatial rotations, spatiotemporal translations and inertial transformations (boosts). This leads to space and time homogeneity and space isotropy, but does not determine their precise realisation completely. This freedom is for example evident from the way boosts act on space and time (e.g., compare the galilean and Poincaré boosts) and through the existence of curvature (e.g., compare Minkowski and de Sitter spacetimes). This leads to the fundamental question [1]:

What are the possible kinematical symmetries of space and time? 
A partial mathematical answer to this question is the classification of kinematical Lie algebras up to isomorphism, which started with the seminal work of Bacry and Lévy-Leblond [1] and of Bacry and Nuyts [2], who classified kinematical algebras (with space isotropy) in the classical case of $3+1$ dimensions, and culminated recently with a classification in arbitrary dimension using techniques in deformation theory [3-5]. The reason this classification is only a partial answer is that the isomorphism type of the Lie algebra is too coarse an invariant: it does not determine uniquely the geometric realisation of the Lie algebra. The ur-example is the Lorentz Lie algebra $\mathfrak{s o}(D+1,1)$, which acts transitively and isometrically both on de Sitter spacetime and on hyperbolic space in $D+1$ dimensions, and, in what is possibly a new twist on an old tale, we will see that it also acts transitively on a carrollian spacetime of the same dimension.

The first step towards a complete answer to the fundamental question was taken already in the original paper [1] of Bacry and Lévy-Leblond. Although restricted to $3+1$ dimensions and to spacetimes admitting parity and time-reversal transformations, they already distinguish between the abstract Lie algebras and their geometric realisations on homogeneous spacetimes, arriving at a list of eleven possible kinematics. Our more refined analysis in this paper reduces that list to ten, since the para-galilean and static kinematical Lie algebras lead to isomorphic homogeneous aristotelian spacetimes. In addition, we drop the requirement of parity or time-reversal symmetries and we work in arbitrary (positive) dimension $D+1$.

More precisely, in this paper we give a more complete answer to the fundamental question by classifying the geometric realisations of kinematical Lie algebras on simplyconnected homogeneous spacetimes. The classification we present in this paper, while encompassing the classical geometries like (anti) de Sitter, Minkowski, galilean and carrollian spacetimes and providing a way to systematically understand their relations, will also uncover new spacetimes and their connections to the ones just mentioned.

By comparison to the seminal works [1,2], the novelty of our approach is predicated on the following features:

Geometry: although our study departs from the classification of kinematical (and aristotelian) Lie algebras, our work focuses on classifying homogeneous spaces. This is an important distinction because, as we will see (see, e.g., table 15), the same Lie algebra may act transitively on different spacetimes, while different Lie algebras may act transitively on the same spacetime. For example, it follows from a careful analysis that despite there being a richer set of isomorphism classes of kinematical Lie algebras for $D=3$ than for $D>3$, there are no uniquely four-dimensional kinematical homogeneous spacetimes.

Parity and time reversal: we relax the "by no means compelling" restriction of parity and time-reversal invariance of the homogeneous spacetimes and, in so doing, we uncover novel kinematical spacetimes. The possibility of dropping this restriction was already noted in [1] and was dropped at the Lie algebraic level in [2], where it was observed that every kinematical Lie algebra (with $D=3$ ) acts transitively on some four-dimensional homogeneous spacetime, but they stopped short of investigating the 
precise relationship between the Lie algebras and the homogeneous spacetimes. As we will see, the relation is rather intricate, as illustrated, for example, in table 15.

Dimension: we go beyond (both above and below) $3+1$ dimensions. Our analysis is valid for any (positive) spacetime dimension $D+1$. Whereas the case of $3+1$ dimensions turns out (after some detailed analysis) to be already generic, in low dimensions $(D \leq 2)$ the situation is more involved and the classification of twoand three-dimensional homogeneous spacetimes differs markedly from that in generic dimension.

Homogeneous kinematical spacetimes are known to play an important rôle in physics. For example Minkowski and (anti) de Sitter spacetimes are crucial in high energy physics, general relativity and cosmology and many other spacetimes arise from them via limits. These limits often induce contractions of their symmetry algebras. It is therefore not surprising that they too arise in various areas of physics. Lie groups and their homogeneous spaces have a plethora of applications (representation theory, coadjoint orbits,...) and are ubiquitous in physics (classical mechanics, hydrodynamics, cosmology, ... ) and one might, therefore, hope that the same is true for our (novel) spacetimes. These might be hard to foresee at present, after all in [1] it was deemed that "the physical interest of [the Carroll groups] is very much reduced", whereas there is no lack of interest in the Carroll group at present. Nevertheless we wish to highlight interesting applications and areas where kinematical spacetimes and their Lie algebras do arise:

Gauge/gravity duality: Anti de Sitter spacetime (AdS) has been the focus of substantial interest due to the conjectured duality to conformal field theory (CFT) [6]. Since AdS is a kinematical spacetime it might be tempting to see if this relation generalises to possible non-AdS/non-CFT dualities, especially since many of the kinematical spacetimes naturally arise as limits of AdS. Indeed, one of the main motivations for the study of kinematical spacetimes is to explore possible new holographies beyond AdS/CFT. Kinematical spacetimes might arise either as bulk spacetimes, similarly to Schrödinger [7, 8] and Lifshitz [9] spacetimes, or as geometries to which bulk theories couple $[10,11]$. For reviews see, e.g., [12, 13].

Furthermore, homogeneous spaces have already shown their usefulness in holographic setups beyond AdS/CFT, see e.g., [14-18] and therefore one might anticipate further interesting results based on analyses of our novel spacetimes.

Condensed matter: besides holographic applications, non-relativistic spaces and especially Newton-Cartan geometry has been a useful tool in the construction of effective field theories for quantum Hall states [19-21] where coset constructions provide a systematic tool to implement symmetries (see, e.g., [22]). It is often the case that the underlying symmetries are given by the centrally extended Galilei algebra: that is, the Bargmann algebra and in a follow-up paper we will present a classification of homogeneous spacetimes of Lie algebras generalising the Bargmann algebra. 
Cosmology: the classification presented in this paper can be understood as an extension of the classification of maximally symmetric lorentzian spacetimes. When imposing the restriction of the existence of an invariant lorentzian metric on the spacetimes we indeed recover the well known result that they consist of the (anti) de Sitter and Minkowski spacetimes. ${ }^{1}$ Dropping the assumption of the existence of a lorentzian metric, but keeping the same amount of symmetry and especially space isotropy and space and time homogeneity, basically leads to this more general classification. Like the lorentzian geometries, the other spacetimes represent empty universes and might be relevant for approximations of the de Sitter universe, see e.g., [23].

Ultra-relativistic structures: the "absolute space" limit of lorentzian spacetimes leads to carrollian structures, where the metric is degenerate and space is absolute. It is closely related to the strong coupling limit of general relativity [24], arises as a limit of duality invariant theories [25] and has recently been connected to asymptotically flat spacetimes [26]. The non-flat carrollian spacetimes have attracted less attention but might lead to interesting generalisations.

New theories: Lie algebras and their associated spacetimes are a natural starting point for the construction of novel theories. Gauging the symmetries of various kinematical algebras (and their central extensions) has been investigated in recent years, e.g., in [27-29]. Furthermore, a thorough analysis of connections and dynamical trajectories of some kinematical structures has been undertaken [30, 31].

A distinguished class of theories are those which are governed by an action principle. Here $2+1$ dimensions seem especially fruitful, where theories based on Chern-Simons actions and (generalisations of) kinematical algebras have been constructed [29, 3237]. Following the seminal work of [38], recently galilean [39-41] and carrollian [39, 42] electrodynamics and gravity [29, 43] and their possible action principles have been investigated. Given the new results of this work there remains much room for further explorations.

Having motivated our interest on kinematical Lie algebras and their spacetimes, we now give a somewhat detailed overview of the contents of the paper.

\subsection{Overview of results}

One of the main results in this paper is the classification of (simply-connected) spacetimes which extend the class of maximally symmetric lorentzian manifolds familiar from general relativity. In this section we will review what is already known about this classification and will summarise how the results obtained in this paper complete that picture. Although in the paper we also consider spacetimes which are unique to two and three dimensions, the bulk of the discussion in this overview section will focus on those spacetimes which exist in all dimensions; although we will mention at the end how these results are modified in low dimension.

\footnotetext{
${ }^{1}$ In general, the Friedmann-Lemaître-Robertson-Walker cosmologies do not correspond to homogeneous spaces since they are only homogeneous in space and not in time.
} 
Our starting points are the de Sitter spacetimes. These are lorentzian spacetimes which are locally isometric to quadric hypersurfaces in pseudo-euclidean spaces. Concretely, de Sitter and anti de Sitter spacetimes in $D+1$ dimensions with radius of curvature $R$ are locally isometric, respectively, to the quadrics

$$
x_{1}^{2}+x_{2}^{2}+\cdots+x_{D}^{2}+x_{D+1}^{2}-x_{D+2}^{2}=R^{2} \quad \text { in } \mathbb{R}^{D+1,1},
$$

and

$$
x_{1}^{2}+x_{2}^{2}+\cdots+x_{D}^{2}-x_{D+1}^{2}-x_{D+2}^{2}=-R^{2} \quad \text { in } \mathbb{R}^{D, 2} .
$$

More precisely, the de Sitter spacetimes are the simply-connected universal covers of these quadrics. Taking the limit $R \rightarrow \infty$ is equivalent to the zero curvature limit in which we recover Minkowski spacetime: the real affine space $\mathbb{A}^{D+1}$ with a metric which, when expressed relative to affine coordinates, is given by

$$
d x_{1}^{2}+d x_{2}^{2}+\cdots+d x_{D}^{2}-c^{2} d x_{D+1}^{2},
$$

where we have introduced the speed of light $c$. We may now take the non-relativistic limit (on the co-metric) in which $c \rightarrow \infty$ or the ultra-relativistic limit in which $c \rightarrow 0$. In the former case we arrive at the galilean spacetime, whereas in the latter, we arrive at the carrollian spacetime $[39,44]$. These spacetimes are no longer lorentzian: the (co-)metric becomes degenerate in the limit, leading to a galilean and a carrollian structure, respectively. This is not to say that on the underlying manifold of such spacetimes one could not define a lorentzian metric, but simply that any such metric would not be invariant under the kinematical symmetries of the spacetime, in the way that the Minkowski metric is Poincaré invariant.

Geometrically, the ultra- and non-relativistic limits can be understood in terms of what they do to the light cone present in the tangent space at any point in Minkowski spacetime, as depicted in figure 1, where we see that the light cone collapses to a timelike line or a spacelike hyperplane in the ultra- and non-relativistic limits, respectively. Since the tangent spaces in a lorentzian manifold are lorentzian vector spaces containing their own light cones, we can consider these limits not just for Minkowski spacetime, but for any lorentzian manifold. In particular, we can do this with the de Sitter spacetimes. The non-relativistic limits of the de Sitter spacetimes are the galilean (anti) de Sitter spacetimes (also known as the Newton-Hooke or non-relativistic cosmological spacetimes) [1, 45-47], whereas the ultra-relativistic limits are the carrollian (anti) de Sitter spacetimes (also known as paraeuclidean and para-Minkowski spacetimes) [1]. As in the case of Minkowski spacetimes, the limiting spacetimes are no longer lorentzian, but have galilean and carrollian structures, respectively.

Just like Minkowski spacetime is the zero-curvature limit of the de Sitter spacetimes, the galilean (resp. carrollian) spacetime can be obtained as a zero-curvature limit of the galilean (resp. carrollian) (anti) de Sitter spacetimes. These spacetimes are not lorentzian and thus, in contrast to the Minkowski case, the curvature being taken to zero is not the Riemann curvature of a (non-existing) Levi-Civita connection. Indeed, as we will see, these spacetimes are homogeneous spaces of kinematical Lie groups and as homogeneous 

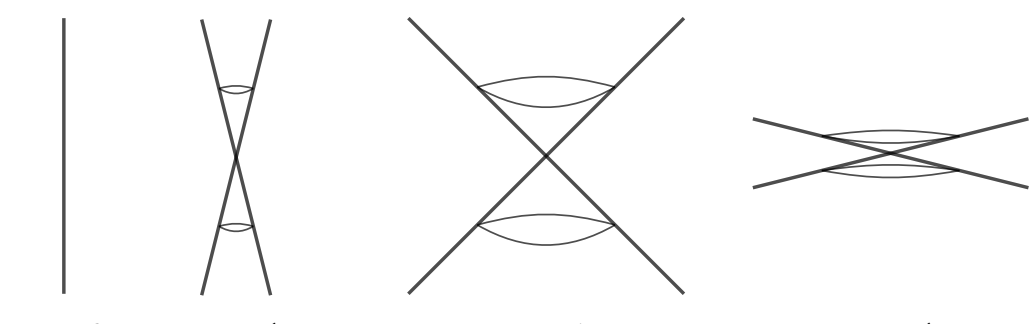

$c=0$

$c \ll 1$

$c=1$

$c \gg 1$

$c=\infty$

Figure 1. Effect on light cone of ultra- (left) and non-relativistic (right) limits.

spaces they are reductive and symmetric and hence in possession of a canonical torsion-free invariant connection. It is that connection whose curvature is being sent to zero.

The galilean and carrollian symmetric spaces can also arise as limits of the riemannian symmetric spaces. The physical interpretation of the riemannian analogues of the non- and ultra-relativistic limits of euclidean space to galilean and carrollian spacetimes, respectively, is not so clear. There is no longer a light cone and hence no longer a privileged timelike direction. Nevertheless we may choose any direction (all are equivalent, since the riemannian symmetric spaces are isotropic) and rescale the metric along that direction or along the perpendicular plane, and in this way arrive at the galilean and carrollian spacetimes. Neither the clock one-form in the galilean spacetime nor the invariant vector field in the carrollian spacetime are actually induced in the limit, so they have neither a minkowskian nor a euclidean preferred interpretation.

The resulting picture (incomplete at this stage) is summarised in figure 2 , where $\mathbb{M}$, $C$ and $G$ stand for Minkowski, carrollian and galilean spacetimes, respectively; $\mathbb{E}, \mathbb{H}$ and $\mathbb{S}$ for euclidean space, hyperbolic space and the round sphere, respectively; (A)dS for (anti) de Sitter spacetimes; and (A)dSG and (A)dSC for the galilean and carrollian (anti) de Sitter spacetimes, respectively. Diagonal arrows are flat limits, whereas horizontal and vertical arrows are, respectively, ultra- and non-relativistic limits. Notice that whereas the analogue of the ultra-relativistic limit of hyperbolic space is carrollian AdS, the analogue of the nonrelativistic limit is galilean $\mathrm{dS}$, and vice versa for the round sphere.

One of the main results in this paper is to complete this picture to the one illustrated by figure 3 (and also figures 4 and 5 for lower dimension) which includes the (simplyconnected) homogeneous spacetimes of all the kinematical Lie groups, with the exception of the riemannian maximally symmetric spaces, whose inclusion might obscure more than enlighten. Much of this paper is devoted to explaining that picture and describing how to arrive at it, but for now let us describe briefly its salient features:

- The galilean de Sitter spacetime dSG is actually the unique symmetric point in a one-parameter family $\mathrm{dSG}_{\gamma}$, with $\gamma \in[-1,1]$, of reductive homogeneous spaces, distinguished by the torsion of the canonical connection, which vanishes at the symmetric point $\gamma=-1$.

- Similarly, the galilean anti de Sitter spacetime AdSG is the unique symmetric point in a one-parameter family $\operatorname{AdSG}_{\chi}$, with $\chi \geq 0$, of reductive homogeneous spaces, distin- 

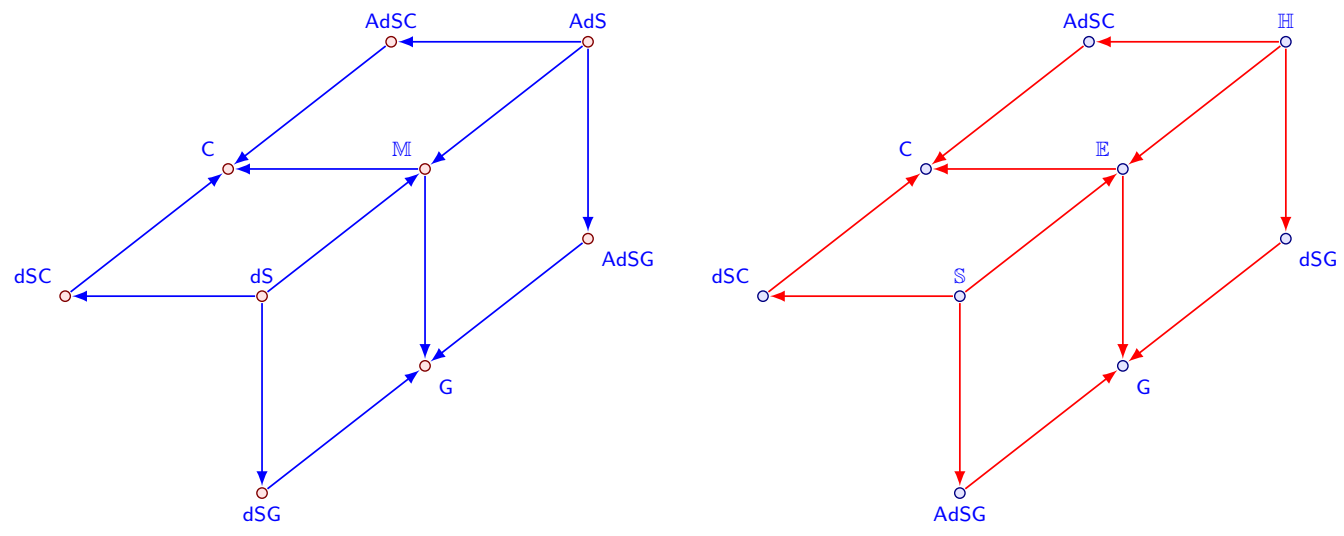

Figure 2. Maximally symmetric spaces and their limits: non-relativistic (vertical), ultra-relativistic (horizontal) and flat (diagonal).

guished by the torsion of the canonical connection, which vanishes at the symmetric point $\chi=0$. Moreover, $\mathrm{AdSG}_{\infty}:=\lim _{\chi \rightarrow \infty} \mathrm{AdSG}_{\chi}=\mathrm{dSG}_{1}$.

- There is a non-reductive homogeneous spacetime $\mathrm{LC}$ of $\mathrm{SO}(D+1,1)$ with an invariant carrollian structure admitting a limit to the carrollian spacetime. This will be shown to be isomorphic to the (future) light cone in Minkowski spacetime in one dimension higher, hence the notation.

- There are several aristotelian homogeneous spacetimes, which are spacetimes without boosts:

- the static affine spacetime $\mathrm{S}$ to which all other spacetimes have limits,

- a torsional aristotelian spacetime TS corresponding to the group manifold of a non-abelian solvable Lie group, and

- the Einstein static universe $\mathbb{R} \times \mathbb{S}^{D}$ and its hyperbolic version $\mathbb{R} \times \mathbb{H}^{D}$, which do not arise from kinematical groups for $D \neq 3$.

In addition, although not depicted in figure 3, there are also the riemannian maximally symmetric spaces (sphere $\mathbb{S}^{D+1}$, hyperbolic $\mathbb{H}^{D+1}$ and euclidean $\mathbb{E}^{D+1}$ ), whose rôle as spacetimes, due to their compact "boosts", is questionable.

When $D=2$, figure 3 is slightly modified to figure 4 . From that figure we see that all that happens now is that there is a new aristotelian spacetime (A24) and a new two-parameter family $\left(\mathrm{S} 12_{\gamma, \chi}\right)$ of galilean spacetimes interpolating between the torsional galilean (anti) de Sitter spacetimes. There are limits from every spacetime in that family to the galilean spacetime. This figure also omits the riemannian maximally symmetric spaces.

Finally, when $D=1$, figure 3 is also modified. Now there are accidental pairwise isomorphisms between some of the symmetric spacetimes due to the possibility of redefining what we mean by space and time. In addition there are new two-dimensional spacetimes with no discernible structure: two spacetimes (S17 and S18) and two continua (S19 $\chi$ and $\left.\mathrm{S} 20_{\chi}\right)$. The resulting picture is depicted in figure 5 . 


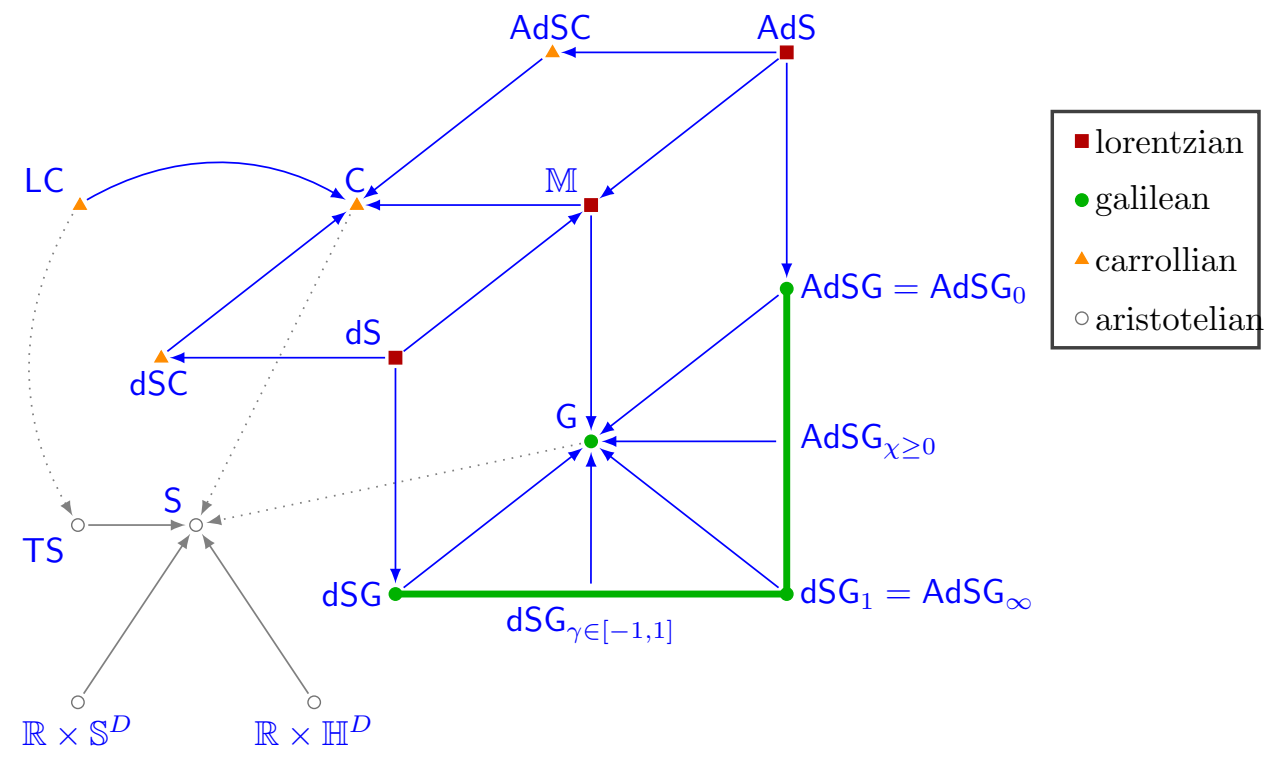

Figure 3. Homogeneous spacetimes in dimension $D+1 \geq 4$ and their limits.

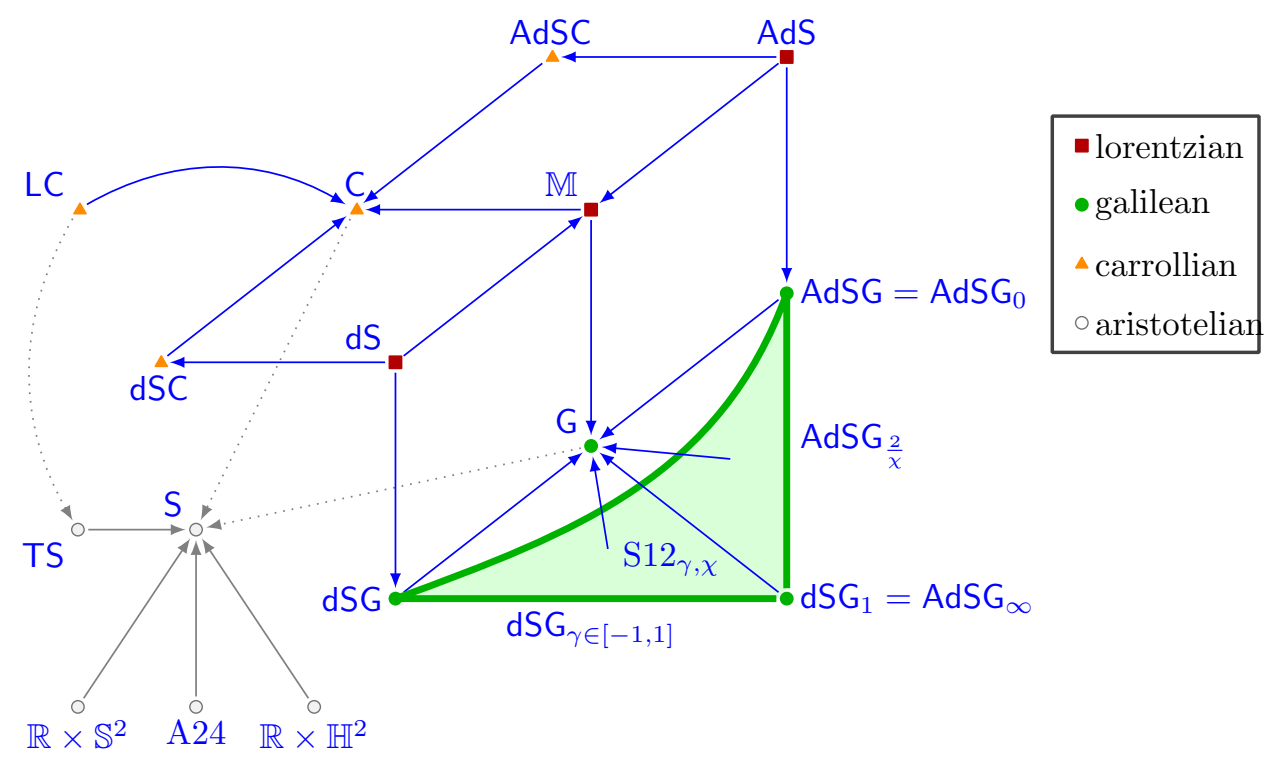

Figure 4. Three-dimensional homogeneous spacetimes and their limits.

\subsection{Organisation of the paper}

The rest of this paper is organised as follows.

Section 2 contains the basic definitions and a summary of the main results in the paper. Readers who are pressed for time should perhaps read that section and then skip to section 6. In section 2.1 we define the main objects of interest: kinematical Lie algebras, their homogeneous spacetimes and their infinitesimal description in terms of Lie pairs, relegating to appendix B a more careful treatment including the proof that (geometrically realisable, effective) Lie pairs are in one-to-one correspondence with simply-connected homogeneous spaces. In section 2.2 we summarise the main results and list the resulting isomorphism 


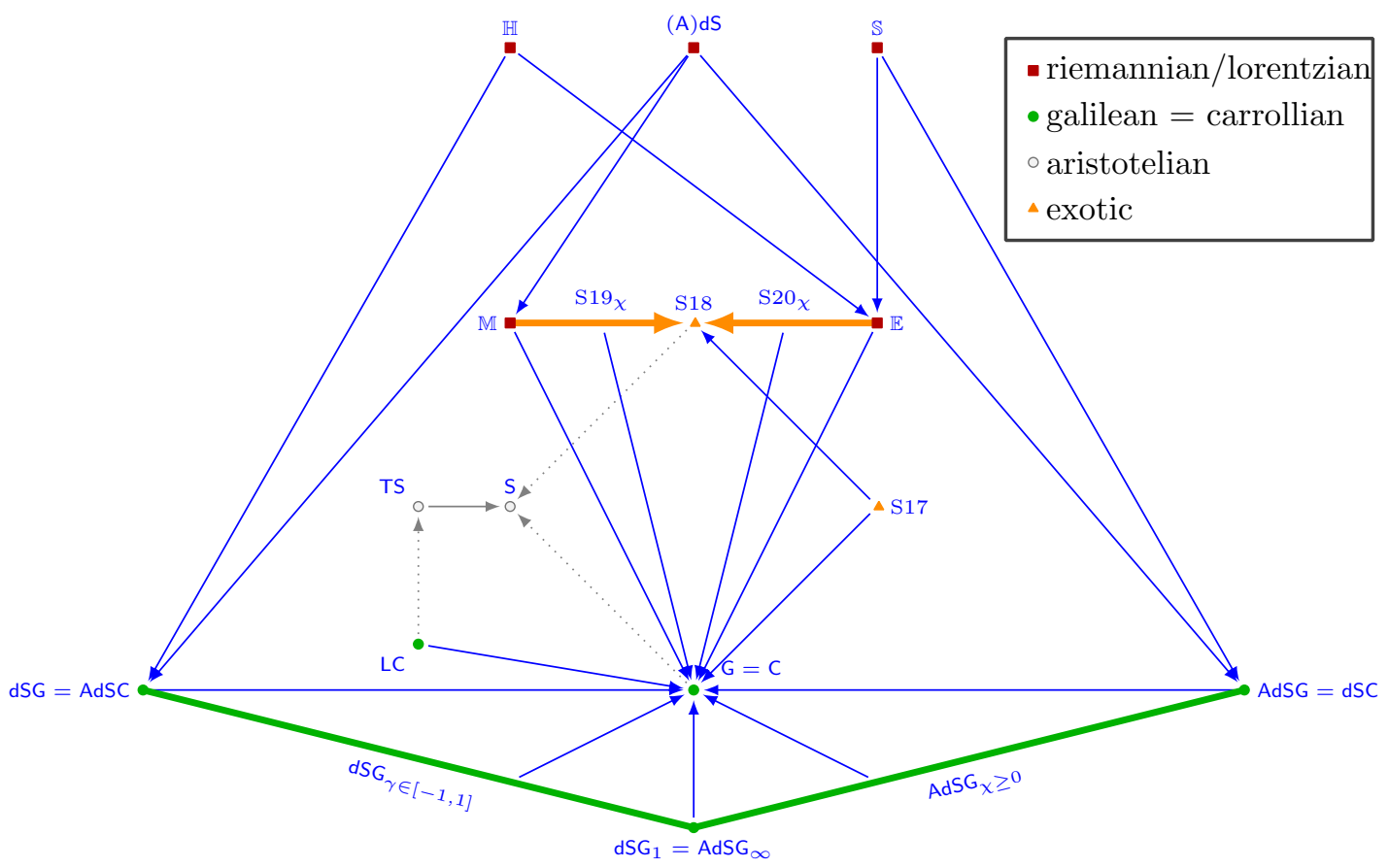

Figure 5. Two-dimensional homogeneous spacetimes and their limits.

classes of simply-connected kinematical and aristotelian homogeneous spacetimes in tables 1 and 2 .

Sections 3 and 4 contains the details leading up to table 1 . (Table 2 is the result of the classification of aristotelian Lie algebras in appendix A.) In section 3 we classify the isomorphism classes of kinematical Lie pairs. This is achieved by going one by one through the isomorphism classes of kinematical Lie algebras and determining for each one the possible Lie pairs up to isomorphism. We do this in turn for generic kinematical Lie algebras in $D \geq 3$ in section 3.1, kinematical Lie algebras unique to $D=3$ in section 3.2, kinematical Lie algebras in $D=2$ in 3.3 and finally those in $D=1$ in section 3.4. The classification of kinematical Lie algebras are summarised in tables 4, 6, 8 and 11 and that of their corresponding Lie pairs are summarised in tables 5, 7, 9 and 12. Some Lie pairs in $D \leq 2$ can be seen to be the low-dimensional avatars of some Lie pairs which exist for all $D$. Tables 10 and 13 describe this correspondence. In section 4 we select from the Lie pairs obtained in section 3 those which are effective and then show, using a variety of methods, that all effective Lie pairs are geometrically realisable. The end result of this section and one of the main results of this work is the list of simply-connected homogeneous kinematical spacetimes in table 1 .

The classification of homogeneous spacetimes provides the "objects" in figure 3. The "arrows" between these objects is provided by limits. In section 5 we flesh out figures 3,4 and 5 by exhibiting the limits relating different spacetimes in tables 1 and 2 .

Section 6 starts the geometric study of the homogeneous spacetimes in tables 1 and 2 . In section 6.1 we review some basic notions like reductivity, symmetry, the linear isotropy representation and the invariant structures of interest: lorentzian, riemannian, galilean, 
carrollian and aristotelian. In section 6.2 we briefly review the notion of an invariant (affine) connection on a homogeneous space and explore how properties (or absence of) canonical invariant connections help us characterise the spacetimes. In section 6.3 we list the resulting spacetimes dividing them into classes depending on the whether they are nonreductive, flat symmetric, non-flat symmetric and reductive torsional. Table 16 summarises these results for the homogeneous spacetimes in tables 1 and 2 .

Finally, section 7 we offer some conclusions and point to further work. In addition, there are two appendices in the paper. In appendix A we classify aristotelian Lie algebras and in appendix B we prove that our infinitesimal approach actually classifies simplyconnected homogeneous spacetimes.

\subsection{Reader's guide}

It is our intention that this paper should be useful to the community, but realise that there is a risk that the results are hard to extract from the details of how we arrived at them. We have therefore tried to write the paper in a way that a reader who is happy to believe the classification can reach it quickly without having to wade through the details of how we arrived at it. The main information content of this paper is contained in sections 2 and 6 and a reader who is pressed for time should perhaps concentrate on those two sections at a first reading. In particular, tables 1 and 2 contain the list of simply-connected homogeneous kinematical and aristotelian spacetimes and table 16 lists their basic geometrical properties: whether they are reductive/symmetric/affine, whether they admit parity and/or time reversal symmetry, the type of invariant structure that they possess (if any): lorentzian, riemannian, galilean, carrollian or aristotelian, and, for the reductive examples, whether the canonical invariant connection is flat and/or torsionfree. In addition, figures 3, 4 and 5 illustrate limits between the spacetimes and it too contains useful information. Busy readers should be able to read the introduction and section 2 and then skip to section 6 where we discuss some geometrical properties of the homogeneous spacetimes.

Many of the tables and figures contain hyperlinks to ease navigation. Let us explain here how to use them. The spacetimes in figures 3,4 and 5 as well as our summary table of the properties, table 16, are hyperlinked to table 1 and 2 which provide the explicit kinematical and aristotelian spacetimes. Every row in these tables starts with a label corresponding to one of the kinematical spacetimes. These labels are hyperlinked to the corresponding label in table 14. That table lists for each spacetime the corresponding effective Lie pairs. These Lie pairs are themselves hyperlinked to the relevant tables of Lie pairs: table 5 for $D \geq 3$, Table 9 for $D=2$ and table 12 for $D=1$. Those tables also contain the information of the isomorphism class of kinematical Lie algebra associated to that Lie pair and the label of the Lie algebra is hyperlinked to the relevant tables of kinematical Lie algebras: table 4 for $D \geq 3$, table 8 for $D=2$ and table 11 for $D=1$. In addition, table 15 shows which homogeneous kinematical spacetimes are associated with which kinematical Lie algebra.

For example, if we click on LC in figure 3 we are taken to table 1. Clicking on S16 in table 1 , we are taken to table 14 where we see that it can be described by several Lie pairs, depending on dimension: LP17 $(D \geq 3), \operatorname{LP} 45(D=2)$ or LP79 $(D=1)$. Clicking, say, 
on LP17, we are taken to table 5, where we see that it comes from Lie algebra LA11 and clicking on LA11 we are taken to table 4 , where we see that it corresponds to $\mathfrak{s o}(D+1,1)$ with the Lie brackets given there.

\section{Kinematical spacetimes}

The well-known spacetimes in figure 2 are all symmetric homogeneous spaces of kinematical Lie groups. In this section we will review this description.

\subsection{Basic definitions}

Before we properly define the main objects of interest, let us motivate our definitions and summarise the philosophy of the construction. By definition, a kinematical Lie algebra has the same dimension as the Lie algebra of isometries of a maximally symmetric riemannian/lorentzian spacetime, and it consists of spatial rotations and additional generators we call boosts and (space and time) translations. However before we specify how the kinematical Lie algebra acts on the spacetime, we cannot make a precise identification of which generators are boosts and/or translations. This is the reason to refine the discussion from the kinematical Lie algebras to the homogeneous spacetimes. The spacetimes are constructed in such a way that the stabiliser of any point contains the rotations about that point. This spatial isotropy implies that all invariant tensors are isotropic (i.e., $\mathfrak{s o}(D)$ rotationally invariant). The vectorial generators in the stabiliser are interpreted as boosts, whereas the additional generators are interpreted as translations. The resulting spacetime is by construction homogeneous, which roughly means that every point of the manifold looks like any other point. We now provide the precise details.

Definition 1. A kinematical Lie algebra (with $D$-dimensional space isotropy) is a real Lie algebra $\mathfrak{k}$ satisfying the following two properties:

1. $\mathfrak{k}$ contains a Lie subalgebra $\mathfrak{r} \cong \mathfrak{s o}(D)$, the Lie algebra of rotations of $D$-dimensional euclidean space; and

2. $\mathfrak{k}$ decomposes as $\mathfrak{k}=\mathfrak{r} \oplus 2 V \oplus S$ as a representation of $\mathfrak{r}$, where $2 V$ are two copies of the $D$-dimensional (vector) irreducible representation of $\mathfrak{s o}(D)$ and $S$ is the onedimensional (scalar) trivial representation of $\mathfrak{s o}(D)$.

By a kinematical Lie group we mean any Lie group whose Lie algebra is a kinematical Lie algebra.

It follows from this definition that we can describe a kinematical Lie algebra explicitly in terms of a basis $J_{a b}=-J_{b a}$ for the rotational subalgebra $\mathfrak{r}, V_{a}^{(i)}$ with $i=1,2$ for the two copies of $V$ and $H$ for $S$. The definition implies that the Lie brackets of $\mathfrak{k}$ in this basis include the following:

$$
\begin{aligned}
{\left[J_{a b}, J_{c d}\right] } & =\delta_{b c} J_{a d}-\delta_{a c} J_{b d}-\delta_{b d} J_{a c}+\delta_{a d} J_{b c} \\
{\left[J_{a b}, V_{c}^{(i)}\right] } & =\delta_{b c} V_{a}^{(i)}-\delta_{a c} V_{b}^{(i)} \\
{\left[J_{a b}, H\right] } & =0
\end{aligned}
$$


and any other Lie brackets are subject only to the Jacobi identity, which implies, in particular, equivariance under $\mathfrak{r}$. It is convenient to relabel $V_{a}^{(1)}$ as $B_{a}$ and $V_{a}^{(2)}$ as $P_{a}$, a notation reminiscent of the boosts and translations in kinematics; although it must be stressed that there is no a priori geometrical interpretation of these generators: they only acquire such an interpretation when we realise them in a spacetime on which the kinematical Lie group acts.

Kinematical Lie algebras have been classified up to Lie algebra isomorphism [1-5]. These classifications are the starting point to the classification of homogeneous spacetimes and hence they will be briefly recalled at the start of sections 3.1, 3.2, 3.3 and 3.4 and contained in tables $4,6,8$ and 11 .

In this paper we obtain a classification of simply-connected homogeneous spacetimes of kinematical Lie groups (up to isomorphism). Not every homogeneous space of a kinematical Lie group is a spacetime, so this requires a definition. Notice that, in particular, a homogeneous spacetime of a kinematical Lie group with $D$-dimensional space isotropy is $(D+1)$-dimensional.

Definition 2. By a homogeneous kinematical spacetime we mean a homogeneous space $M$ of a kinematical Lie group $\mathcal{K}$, satisfying the following properties:

1. $M$ is a connected smooth manifold, and

2. $\mathcal{K}$ acts transitively and locally effectively ${ }^{2}$ on $M$ with stabiliser $\mathcal{H}$, where

3. $\mathcal{H}$ is a closed subgroup of $\mathcal{K}$ whose Lie algebra $\mathfrak{h}$ contains a rotational subalgebra $\mathfrak{r} \cong \mathfrak{s o}(D)$ and decomposes as $\mathfrak{h}=\mathfrak{r} \oplus V$ as an adjoint $\mathfrak{r}$-module, where $V$ is an irreducible $D$-dimensional vector representation of $\mathfrak{s o}(D)$.

It follows that $M$ is $\mathcal{K}$-equivariantly diffeomorphic to $\mathcal{K} / \mathcal{H}$. Within the confines of this paper, and because we will need to refer to them often, we will say that $\mathcal{H}$ and $\mathfrak{h}$ are admissible.

It may be convenient to keep an example in mind, so let us consider the Poincaré Lie group, whose Lie algebra is defined relative to the basis $\left(J_{a b}, B_{a}, P_{a}, H\right)$ by the nonzero Lie brackets

$$
\begin{aligned}
{\left[J_{a b}, J_{c d}\right] } & =\delta_{b c} J_{a d}-\delta_{a c} J_{b d}-\delta_{b d} J_{a c}+\delta_{a d} J_{b c} & & {\left[B_{a}, B_{b}\right]=J_{a b} } \\
{\left[J_{a b}, B_{c}\right] } & =\delta_{b c} B_{a}-\delta_{a c} B_{b} & & {\left[B_{a}, P_{b}\right]=\delta_{a b} H } \\
{\left[J_{a b}, P_{c}\right] } & =\delta_{b c} P_{a}-\delta_{a c} P_{b} & {\left[B_{a}, H\right] } & =P_{a} .
\end{aligned}
$$

This is obtained from the more familiar expression

$$
\begin{aligned}
{\left[J_{\mu \nu}, J_{\rho \sigma}\right] } & =\eta_{\nu \rho} J_{\mu \sigma}-\eta_{\mu \rho} J_{\nu \sigma}-\eta_{\nu \sigma} J_{\mu \rho}+\eta_{\mu \sigma} J_{\nu \rho} \\
{\left[J_{\mu \nu}, P_{\rho}\right] } & =\eta_{\nu \rho} P_{\mu}-\eta_{\mu \rho} P_{\nu},
\end{aligned}
$$

by decomposing $J_{\mu \nu}=\left(J_{a b}, B_{a}:=J_{0 a}\right)$ and $P_{\mu}=\left(P_{a}, H:=P_{0}\right)$, where $\eta_{00}=-1$ and $\eta_{a b}=\delta_{a b}$. Let us take $\mathcal{K}$ to be the Poincaré Lie group and $\mathcal{H}$ the Lorentz subgroup; that

\footnotetext{
${ }^{2}$ See appendix B.1 for the basic definitions, if needed.
} 
is, the (admissible) subgroup generated by the Lie subalgebra spanned by $\left(J_{a b}, B_{a}\right)$. Then $\mathcal{K} / \mathcal{H}$ is diffeomorphic to Minkowski spacetime, as is well known.

Perhaps not so well known is the fact that the Poincaré group admits a second homogeneous spacetime. If we now let $\mathcal{H}^{\prime}$ denote the (again, admissible) subgroup generated by the Lie subalgebra $\mathfrak{h}^{\prime}$ spanned by $\left(J_{a b}, P_{a}\right)$, then $\mathcal{K} / \mathcal{H}^{\prime}$ is diffeomorphic to the carrollian anti de Sitter spacetime (AdSC) (also known as para-Minkowski spacetime), as we will see below. To more easily distinguish between these two homogeneous spacetimes of the Poincaré group, it is convenient to change basis in the Poincaré Lie algebra in such a way that the admissible Lie subalgebra $\mathfrak{h}^{\prime}$ is also spanned by $\left(J_{a b}, B_{a}\right)$. Doing so we arrive at what is often termed the para-Poincaré Lie algebra, with nonzero Lie brackets

$$
\begin{array}{rlrl}
{\left[J_{a b}, J_{c d}\right]} & =\delta_{b c} J_{a d}-\delta_{a c} J_{b d}-\delta_{b d} J_{a c}+\delta_{a d} J_{b c} & & {\left[B_{a}, P_{b}\right]=\delta_{a b} H} \\
{\left[J_{a b}, B_{c}\right]=\delta_{b c} B_{a}-\delta_{a c} B_{b}} & {\left[H, P_{a}\right]} & =B_{a} \\
{\left[J_{a b}, P_{c}\right]} & =\delta_{b c} P_{a}-\delta_{a c} P_{b} & & {\left[P_{a}, P_{b}\right]=J_{a b} .}
\end{array}
$$

Of course, this Lie algebra is isomorphic to the Poincaré Lie algebra, but not in a way which fixes the admissible subalgebra. We see from the above Lie brackets that translations no longer commute, signalling that this spacetime is not flat; although, as we will see, the nonflat connection, which is the canonical Poincaré-invariant connection on this symmetric homogeneous space, is not a metric connection since AdSC does not admit a Poincaréinvariant metric. If it did, by dimension, it would have be maximally symmetric and hence isometric to Minkowski spacetime, where translations do commute.

As we explain in appendix B, the classification of isomorphism classes of simplyconnected homogeneous kinematical spacetimes can be arrived at infinitesimally, by classifying isomorphism classes of (geometrically realisable, effective) kinematical Lie pairs. This too requires a definition.

Definition 3. A (kinematical) Lie pair is a pair $(\mathfrak{k}, \mathfrak{h})$ consisting of a kinematical Lie algebra $\mathfrak{k}$ and an admissible subalgebra $\mathfrak{h}$. Two Lie pairs $\left(\mathfrak{k}_{1}, \mathfrak{h}_{1}\right)$ and $\left(\mathfrak{k}_{2}, \mathfrak{h}_{2}\right)$ are isomorphic if there is a Lie algebra isomorphism $\varphi: \mathfrak{k}_{1} \rightarrow \mathfrak{k}_{2}$ with $\varphi\left(\mathfrak{h}_{1}\right)=\mathfrak{h}_{2}$. A Lie pair $(\mathfrak{k}, \mathfrak{h})$ is effective if $\mathfrak{h}$ does not contain any nonzero ideals of $\mathfrak{k}$. It is said to be (geometrically) realisable if there exists a connected Lie group $\mathcal{K}^{\prime}$ with Lie algebra $\mathfrak{k}^{\prime}$ and a closed Lie subgroup $\mathcal{H}^{\prime}$ with Lie algebra $\mathfrak{h}^{\prime}$ with $\left(\mathfrak{k}^{\prime}, \mathfrak{h}^{\prime}\right)$ isomorphic to $(\mathfrak{k}, \mathfrak{h})$. The homogeneous space $\mathcal{K}^{\prime} / \mathcal{H}^{\prime}$ is said to be a geometric realisation of $(\mathfrak{k}, \mathfrak{h})$.

The relationship between Lie pairs and homogeneous spaces extends the relationship between Lie algebras and Lie groups. Recall that associated with every finite-dimensional Lie algebra $\mathfrak{k}$ there exists a unique (up to isomorphism) simply-connected Lie group whose Lie algebra is isomorphic to $\mathfrak{k}$. A similar correspondence exists between homogeneous spaces and Lie pairs, except that we need to restrict to Lie pairs which are geometrically realisable (existence) and effective (uniqueness). This is explained in detail in appendix B.

In this paper we will classify isomorphism classes of geometrically realisable, effective Lie pairs $(\mathfrak{k}, \mathfrak{h})$, where $\mathfrak{k}$ is a kinematical Lie algebra and $\mathfrak{h}$ an admissible Lie subalgebra. As just explained, this is equivalent to classifying isomorphism classes of simply-connected 
homogeneous kinematical spacetimes. We will actually exploit the classification of kinematical Lie algebras up to isomorphism and in this way fix a kinematical Lie algebra $\mathfrak{k}$ in each isomorphism class and classify isomorphism classes of (geometrically realisable, effective) Lie pairs $(\mathfrak{k}, \mathfrak{h})$ with $\mathfrak{h}$ an admissible subalgebra, with two such Lie pairs $\left(\mathfrak{k}, \mathfrak{h}_{1}\right)$ and $\left(\mathfrak{k}, \mathfrak{h}_{2}\right)$ declared to be isomorphic if there is an automorphism of $\mathfrak{k}$ sending $\mathfrak{h}_{1}$ to $\mathfrak{h}_{2}$.

\subsection{Summary of main results}

The classification is described in sections 3 and 4 , but we think it might be helpful to the reader to collect here already the results. In the tables below we use an abbreviated notation in which we do not write the $\mathfrak{s o}(D)$ indices explicitly. We write $\boldsymbol{J}, \boldsymbol{B}, \boldsymbol{P}$ and $H$ for the generators of the kinematical Lie algebra $\mathfrak{k}$ and write the kinematical Lie brackets of $(2.1)$ as

$$
[\boldsymbol{J}, \boldsymbol{J}]=\boldsymbol{J} \quad[\boldsymbol{J}, \boldsymbol{B}]=\boldsymbol{B} \quad[\boldsymbol{J}, \boldsymbol{P}]=\boldsymbol{P} \quad \text { and } \quad[\boldsymbol{J}, H]=0 .
$$

For $D \neq 2$, any other brackets can be reconstructed unambiguously from the abbreviated expression since there is only one way to reintroduce indices in an $\mathfrak{s o}(D)$-equivariant fashion; that is, using only the $\mathfrak{s o}(D)$-invariant tensors $\delta_{a b}$ and $\epsilon_{a_{1} \cdots a_{D}}$ on the right hand side of the brackets. For example,

$$
\begin{aligned}
& {[H, \boldsymbol{B}]=\boldsymbol{P} \quad \text { stands for } \quad\left[H, B_{a}\right]=P_{a} \quad \text { and }} \\
& {[\boldsymbol{B}, \boldsymbol{P}]=H+\boldsymbol{J} \quad \text { for } \quad\left[B_{a}, P_{b}\right]=\delta_{a b} H+J_{a b} .}
\end{aligned}
$$

In $D=3$ we may also have brackets of the form

$$
[\boldsymbol{P}, \boldsymbol{P}]=\boldsymbol{P} \quad \text { which we take to mean } \quad\left[P_{a}, P_{b}\right]=\epsilon_{a b c} P_{c},
$$

where we employ Einstein's summation convention. Similarly, for $D=2, \epsilon_{a b}$ is rotationally invariant and can appear in Lie brackets. So we will write, e.g.,

$$
[H, \boldsymbol{B}]=\boldsymbol{B}+\widetilde{\boldsymbol{P}} \quad \text { for } \quad\left[H, B_{a}\right]=B_{a}+\epsilon_{a b} P_{b},
$$

et cetera.

Table 1 summarises all the hard work in sections 3 and 4 and lists all the inequivalent geometrically realisable effective Lie pairs $(\mathfrak{k}, \mathfrak{h})$ with $\mathfrak{k}$ a kinematical Lie algebra and $\mathfrak{h}$ an admissible Lie subalgebra. A basis has been chosen in such a way that $\mathfrak{h}$ is spanned by $\left(J_{a b}, B_{a}\right)$. In this way, Lie pairs are uniquely characterised by specifying the Lie brackets of $\mathfrak{k}$ in this basis. The kinematical Lie brackets (2.5) are common to all kinematical Lie algebras, so that we need only specify those Lie brackets which do not involve the rotations. The table is divided into five sections, separated by horizontal rules. From top to bottom, the first four correspond to the lorentzian, riemannian, galilean and carrollian spacetimes. The final section corresponds to "exotic" two-dimensional spacetimes admitting no such structures. Two remarks are in order about this table and both concern the case of $D=1$. Since there are no rotations in $D=1$, in any row where $\boldsymbol{J}$ appears, we are tacitly assuming that we set $\boldsymbol{J}=0$ if $D=1$. Also, some of the $D \geq 1$ spacetimes become accidentally 


\begin{tabular}{|c|c|c|c|c|c|c|c|}
\hline Label & $D$ & & Nonzero Lie brackets in addition to & {$[J, J]=J,[J$} & $\boldsymbol{B}]=\boldsymbol{B},[\boldsymbol{J}, \boldsymbol{P}]=\boldsymbol{P}$ & & Comments \\
\hline S1 & $\geq 1$ & {$[H, \boldsymbol{B}]=-\boldsymbol{P}$} & & {$[\boldsymbol{B}, \boldsymbol{B}]=\boldsymbol{J}$} & {$[\boldsymbol{B}, \boldsymbol{P}]=H$} & & Minkowski (M) \\
\hline S2 & $\geq 2$ & {$[H, \boldsymbol{B}]=-\boldsymbol{P}$} & {$[H, \boldsymbol{P}]=-\boldsymbol{B}$} & {$[\boldsymbol{B}, \boldsymbol{B}]=\boldsymbol{J}$} & {$[\boldsymbol{B}, \boldsymbol{P}]=H$} & {$[P, P]=-J$} & de Sitter (dS) \\
\hline S3 & $\geq 1$ & {$[H, \boldsymbol{B}]=-\boldsymbol{P}$} & {$[H, \boldsymbol{P}]=\boldsymbol{B}$} & {$[\boldsymbol{B}, \boldsymbol{B}]=\boldsymbol{J}$} & {$[\boldsymbol{B}, \boldsymbol{P}]=H$} & {$[P, P]=J$} & anti de Sitter (AdS) \\
\hline $\mathrm{S} 4$ & $\geq 1$ & {$[H, \boldsymbol{B}]=\boldsymbol{P}$} & & {$[B, B]=-J$} & {$[\boldsymbol{B}, \boldsymbol{P}]=H$} & & euclidean $(\mathbb{E})$ \\
\hline S5 & $\geq 1$ & {$[H, \boldsymbol{B}]=\boldsymbol{P}$} & {$[H, \boldsymbol{P}]=-\boldsymbol{B}$} & {$[B, B]=-J$} & {$[\boldsymbol{B}, \boldsymbol{P}]=H$} & {$[\boldsymbol{P}, \boldsymbol{P}]=-\boldsymbol{J}$} & sphere $(\mathbb{S})$ \\
\hline $\mathrm{S} 6$ & $\geq 1$ & {$[H, \boldsymbol{B}]=\boldsymbol{P}$} & {$[H, \boldsymbol{P}]=\boldsymbol{B}$} & {$[B, B]=-J$} & {$[\boldsymbol{B}, \boldsymbol{P}]=H$} & {$[P, P]=J$} & hyperbolic ( $\mathbb{H})$ \\
\hline S7 & $\geq 1$ & {$[H, \boldsymbol{B}]=-\boldsymbol{P}$} & & & & & galilean (G) \\
\hline S8 & $\geq 1$ & {$[H, \boldsymbol{B}]=-\boldsymbol{P}$} & {$[H, P]=-\boldsymbol{B}$} & & & & galilean de Sitter $\left(\mathrm{dSG}=\mathrm{dSG}_{\gamma=-1}\right)$ \\
\hline $\mathrm{S} 9_{\gamma}$ & $\geq 1$ & {$[H, \boldsymbol{B}]=-\boldsymbol{P}$} & {$[H, \boldsymbol{P}]=\gamma \boldsymbol{B}+(1+\gamma) \boldsymbol{P}$} & & & & torsional galilean de Sitter $\mathrm{dSG}_{\gamma \in(-1,1]}$ \\
\hline S10 & $\geq 1$ & {$[H, \boldsymbol{B}]=-\boldsymbol{P}$} & {$[H, \boldsymbol{P}]=\boldsymbol{B}$} & & & & galilean anti de Sitter $\left(\operatorname{AdSG}=\operatorname{AdSG}_{\chi=0}\right)$ \\
\hline $\mathrm{S} 11_{\chi}$ & $\geq 1$ & {$[H, \boldsymbol{B}]=-\boldsymbol{P}$} & {$[H, \boldsymbol{P}]=\left(1+\chi^{2}\right) \boldsymbol{B}+2 \chi \boldsymbol{P}$} & & & & torsional galilean anti de Sitter $\mathrm{AdSG}_{\chi>0}$ \\
\hline $\mathrm{S} 12_{\gamma, \chi}$ & 2 & {$[H, \boldsymbol{B}]=-\boldsymbol{P}$} & {$[H, \boldsymbol{P}]=(1+\gamma) \boldsymbol{P}-\chi \widetilde{\boldsymbol{P}}+\gamma \boldsymbol{B}-\chi \widetilde{\boldsymbol{B}}$} & & & & $\gamma \in[-1,1), \chi>0$ \\
\hline S13 & $\geq 2$ & & & & {$[\boldsymbol{B}, \boldsymbol{P}]=H$} & & carrollian (C) \\
\hline S14 & $\geq 2$ & & {$[H, P]=-\boldsymbol{B}$} & & {$[\boldsymbol{B}, \boldsymbol{P}]=H$} & {$[\boldsymbol{P}, \boldsymbol{P}]=-\boldsymbol{J}$} & carrollian de Sitter (dSC) \\
\hline S15 & $\geq 2$ & & {$[H, P]=\boldsymbol{B}$} & & {$[\boldsymbol{B}, \boldsymbol{P}]=H$} & {$[P, P]=J$} & carrollian anti de Sitter (AdSC) \\
\hline S16 & $\geq 1$ & {$[H, \boldsymbol{B}]=\boldsymbol{B}$} & {$[H, P]=-\boldsymbol{P}$} & & {$[\boldsymbol{B}, \boldsymbol{P}]=H+\boldsymbol{J}$} & & carrollian light cone (LC) \\
\hline S17 & 1 & {$[H, B]=-P$} & & & {$[B, P]=-H-2 P$} & & \\
\hline S18 & 1 & {$[H, B]=H$} & & & {$[B, P]=-P$} & & \\
\hline $\mathrm{S} 19_{\chi}$ & 1 & {$[H, B]=(1+\chi) H$} & & & {$[B, P]=(1-\chi) P$} & & $x>0$ \\
\hline $\mathrm{S} 20_{\chi}$ & 1 & {$[H, B]=-P$} & & & {$[B, P]=-\left(1+\chi^{2}\right) H-2 \chi P$} & & $\chi>0$ \\
\hline
\end{tabular}

Table 1. Simply-connected homogeneous $(D+1)$-dimensional kinematical spacetimes. Geometrical properties of the spacetimes are provided in table 16.

pairwise isomorphic in $D=1$ : namely, carrollian and galilean, de Sitter and anti de Sitter, carrollian dS and galilean AdS, and carrollian AdS and galilean dS. This explains why we write $D \geq 2$ for carrollian, de Sitter, and carrollian (anti) de Sitter. In this way no two rows are isomorphic and hence every row in the table specifies a unique isomorphism class of simply-connected homogeneous kinematical spacetime. Perhaps it bears repeating that, as mentioned already in the introduction, the galilean (A)dS spacetimes are often called the Newton-Hooke spacetimes.

Table 2 lists the Lie pairs corresponding to the aristotelian spacetimes. Many of them arise from non-effective Lie pairs $(\mathfrak{k}, \mathfrak{h})$ with $\mathfrak{k}$ a kinematical Lie algebra. If such a pair is not effective, it means that $\mathfrak{h}$ contains a nonzero ideal of $\mathfrak{k}$. For $\mathfrak{k}$ a kinematical Lie algebra, this cannot be other than the span $\mathfrak{b}$ of the $\left(B_{a}\right)$, assuming they do form an ideal. When this is the case, we may quotient both $\mathfrak{k}$ and $\mathfrak{h}$ by the ideal $\mathfrak{b}$ and arrive at an effective (by construction) Lie pair $(\mathfrak{k} / \mathfrak{b}, \mathfrak{h} / \mathfrak{b})=(\mathfrak{a}, \mathfrak{r})$, where $\mathfrak{a}=\mathfrak{k} / \mathfrak{b}$ is an aristotelian Lie algebra (see appendix A) and $\mathfrak{r}=\mathfrak{h} / \mathfrak{b}$ is a rotational subalgebra of $\mathfrak{a}$.

To fix ideas, let us consider the example of the galilean algebra $\mathfrak{g}$. This is the kinematical Lie algebra with nonzero Lie brackets $[H, \boldsymbol{B}]=-\boldsymbol{P}$ in addition to those in (2.5). As we will see, there are two isomorphism classes of Lie pairs associated to $\mathfrak{g}$. If we choose bases for $\mathfrak{g}$ so that in both cases the admissible subalgebra is the span of $\left(J_{a b}, B_{a}\right)$, then the two Lie pairs are described by the following Lie brackets for $\mathfrak{g}$ in addition to those in (2.5):

- $[H, \boldsymbol{B}]=-\boldsymbol{P}$, which is the standard galilean spacetime, and

- $[H, P]=\boldsymbol{B}$.

The latter Lie pair is not effective because $\mathfrak{h}$ contains the ideal $\mathfrak{b}$ spanned by the $\left(B_{a}\right)$. If we quotient this Lie pair by $\mathfrak{b}$, which boils down to discarding any $B_{a}$ in the Lie brackets, we see that now $[H, \boldsymbol{P}]=0$ and we arrive at the static aristotelian Lie algebra. 


\begin{tabular}{|l|c|r|l|}
\hline Label & $D$ & Nonzero Lie brackets in addition to $[\boldsymbol{J}, \boldsymbol{J}]=\boldsymbol{J}$ and $[\boldsymbol{J}, \boldsymbol{P}]=\boldsymbol{P}$ & \multicolumn{1}{c|}{ Comments } \\
\hline A21 & $\geq 0$ & & static (S) \\
A22 & $\geq 1$ & {$[H, \boldsymbol{P}]=\boldsymbol{P}$} & torsional static (TS) \\
A23 & $\geq 2$ & {$[\boldsymbol{P}, \boldsymbol{P}]=\boldsymbol{J}$} & $\mathbb{R} \times \mathbb{H}^{D}$ \\
A23 & $\geq 2$ & {$[\boldsymbol{P}, \boldsymbol{P}]=-\boldsymbol{J}$} & $\mathbb{R} \times \mathbb{S}^{D}$ \\
A24 & 2 & {$[\boldsymbol{P}, \boldsymbol{P}]=H$} & \\
\hline
\end{tabular}

Table 2. Simply-connected homogeneous $(D+1)$-dimensional aristotelian spacetimes.

Aristotelian Lie pairs are always geometrically realisable because the rotational subalgebra $\mathfrak{r}$ generates a compact subgroup and compact subgroups are always closed. Furthermore, since $\mathfrak{r}$ is fixed, every aristotelian Lie algebra gives rise to a unique aristotelian Lie pair, so that the classification in appendix A is also a classification of simply-connected aristotelian spacetimes up to isomorphism.

The next two sections contain the classifications leading up to table 1, whereas section 5 contains the details leading up to figures 3,4 and 5. The busy reader may wish to skip them at a first reading and go directly to section 6 where we explore some of the geometrical properties of these spacetimes, culminating in table 16 .

\section{Classification of kinematical Lie pairs}

This section, which can be skipped at a first reading, contains the details of the classification of kinematical Lie pairs $(\mathfrak{k}, \mathfrak{h})$ up to isomorphism, where $\mathfrak{k}$ is a kinematical Lie algebra and $\mathfrak{h}$ is an admissible Lie algebra. At this stage we will not worry about whether the resulting Lie pairs are effective and/or geometrically realisable. The results of this section are collected in tables 5, 7, 9 and 12, which will be the starting point for the analysis in section 4, where we extract from those tables the effective Lie pairs which admit a geometric realisation to arrive at table 1 .

We will simplify the analysis by making use of the classification of kinematical Lie algebras, which we briefly review. Kinematical Lie algebras (with $D$-dimensional space isotropy) have been classified up to Lie algebra isomorphism. In $D=0$ there is a unique one-dimensional Lie algebra, whereas in $D=1$ there are no rotations, so any threedimensional Lie algebra is kinematical. These were classified by Bianchi [48] (see [49] for an English translation) in the context of his classification of three-dimensional homogeneous spaces; although here they will play the rôle of symmetries of two-dimensional homogeneous spaces. The other classic case is $D=3$, where the kinematical Lie algebras were classified by Bacry and Nuyts [2] refining earlier work of Bacry and Lévy-Leblond [1]. The remaining cases $D>3$ and $D=2$ are recent classifications. Following earlier work on the galilean algebra [50], the $D=3$ classification was recovered recently in [3] using methods of deformation theory. These methods were then extended to arrive at the classifications for $D>3[4]$ and $D=2[5]$. These classifications are recalled in this paper and are contained in tables $4,6,8$ and 11 .

The classification of kinematical Lie algebras allows us to fix a kinematical Lie algebra $\mathfrak{k}$ and then consider Lie pairs $(\mathfrak{k}, \mathfrak{h})$, with two such pairs $\left(\mathfrak{k}, \mathfrak{h}_{1}\right)$ and $\left(\mathfrak{k}, \mathfrak{h}_{2}\right)$ being isomorphic 
if there is an automorphism of $\mathfrak{k}$ taking $\mathfrak{h}_{1}$ to $\mathfrak{h}_{2}$. This suggests the following methodology, which is how we will proceed in this paper.

For each kinematical Lie algebra $\mathfrak{k}$ in the classification, we will determine the admissible subalgebras $\mathfrak{h}$ and, if necessary, the action of the automorphism group $A=\operatorname{Aut}(\mathfrak{k})$ on them. We will then pick one representative from each $A$-orbit. Finally, we will change basis (if needed) for $\mathfrak{k}$ so that $\mathfrak{h}$ is always spanned by $J_{a b}$ and $B_{a}$. The resulting Lie pair is then described uniquely by specifying the Lie brackets of $\mathfrak{k}$ in this basis. Furthermore, whenever possible, we choose a basis in which the Lie pair is manifestly reductive.

It follows from the classification of kinematical Lie algebras that there are kinematical Lie algebras which exist for all $D \geq 1$, but there are additional Lie algebras for $D \leq 3$, due to accidents in small dimension: namely, the existence of a rotationally invariant vector product in $D=3$, a rotationally invariant symplectic structure in $D=2$, and the absence of rotations in $D=1$. As explained in [5], the case $D=2$ is special and it is convenient to work with the complexified Lie algebra. The analysis in that case does not embed easily into the general discussion of $D \geq 3$ and therefore we will have to do it separately. Similarly the case $D=1$ is special in that any three-dimensional Lie algebra is kinematical and any one-dimensional subalgebra is admissible. We also treat this case separately. These considerations suggest first treating the generic case (restricted to $D \geq 3$ ), which we do in section 3.1. Then in section 3.2 we consider the additional kinematical Lie algebras which are unique to $D=3$. In section 3.3 we determine the Lie pairs associated to the kinematical Lie algebras in $D=2$. As we will see, there are two kinematical Lie algebras unique to $D=2$ which do not admit any Lie pairs. Then in section 3.4 we consider the Lie pairs associated to the three-dimensional Lie algebras.

\subsection{Lie pairs for $D \geq 3$}

Let us consider those kinematical Lie algebras which have analogs for all $D \geq 3$. (They also have analogs for $D<3$, but we will discuss them again separately in that context.) These are listed in table 4, which is borrowed from [4, table 17] with some small modification. We only list those nonzero Lie brackets in addition to (2.5), which are understood as given. The numbering in this and other tables is somewhat arbitrary, but might help in referring to those Lie algebras which are otherwise nameless. Although we are aware that referring to some of these kinematical Lie algebras by name presupposes a geometrical interpretation of the generators which may or may not be the right interpretation depending on the actual homogeneous space under consideration, we do feel that it helps to orient the reader if we point out to which named Lie algebras (when the name exists) the Lie algebras in table 4 are isomorphic. In this spirit, let us also mention in this and other tables the isomorphism type of the Lie algebra, when known. To this end let us introduce in table 3 some notation for the named Lie algebras appearing in this section.

In discussing the automorphisms of kinematical Lie algebras we will fix the rotational subalgebra $\mathfrak{r}$ and concentrate on automorphisms which are the identity on $\mathfrak{r}$ and in this way focus only on their action on the non-rotational generators. Since automorphisms must commute with the action of the rotations and the vector representation is irreducible (also 


\begin{tabular}{|c|l|}
\hline Notation & \multicolumn{1}{|c|}{ Name } \\
\hline $\mathfrak{s}$ & static \\
$\mathfrak{n}_{+}$ & (elliptic) Newton \\
$\mathfrak{n}_{-}$ & (hyperbolic) Newton \\
$\mathfrak{e}$ & euclidean \\
\hline
\end{tabular}

\begin{tabular}{|c|l|}
\hline Notation & \multicolumn{1}{|c|}{ Name } \\
\hline $\mathfrak{p}$ & Poincaré \\
$\mathfrak{s o}$ & orthogonal \\
$\mathfrak{g}$ & galilean \\
$\mathfrak{c}$ & Carroll \\
\hline
\end{tabular}

Table 3. Notation for Lie algebras.

\begin{tabular}{|c|c|c|c|c|c|}
\hline $\begin{array}{l}\text { LA\# } \\
1\end{array}$ & $\begin{array}{ll} & \cong \\
\mathfrak{s} & \end{array}$ & \multicolumn{3}{|c|}{ Nonzero Lie brackets in addition to $[\boldsymbol{J}, \boldsymbol{J}]=\boldsymbol{J},[\boldsymbol{J}, \boldsymbol{B}]=\boldsymbol{B}$ and $[\boldsymbol{J}, \boldsymbol{P}]=\boldsymbol{P}$} & Comments \\
\hline 2 & $\mathfrak{g}$ & {$[H, \boldsymbol{B}]=\boldsymbol{P}$} & & & \\
\hline $3_{\gamma}$ & & {$[H, \boldsymbol{B}]=\gamma \boldsymbol{B}$} & {$[H, \boldsymbol{P}]=\boldsymbol{P}$} & & $\gamma \in(-1,1)$ \\
\hline 4 & & {$[H, \boldsymbol{B}]=\boldsymbol{B}$} & {$[H, \boldsymbol{P}]=\boldsymbol{P}$} & & \\
\hline 5 & $\mathfrak{n}_{-}$ & {$[H, \boldsymbol{B}]=-\boldsymbol{B}$} & {$[H, \boldsymbol{P}]=\boldsymbol{P}$} & & \\
\hline 6 & & {$[H, \boldsymbol{B}]=\boldsymbol{B}+\boldsymbol{P}$} & {$[H, \boldsymbol{P}]=\boldsymbol{P}$} & & \\
\hline $7_{\chi}$ & & {$[H, \boldsymbol{B}]=\chi \boldsymbol{B}+\boldsymbol{P}$} & {$[H, \boldsymbol{P}]=\chi \boldsymbol{P}-\boldsymbol{B}$} & & $\chi>0$ \\
\hline 8 & $\mathfrak{n}_{+}$ & {$[H, \boldsymbol{B}]=\boldsymbol{P}$} & {$[H, \boldsymbol{P}]=-\boldsymbol{B}$} & & \\
\hline 9 & $\mathfrak{c}$ & & & {$[\boldsymbol{B}, \boldsymbol{P}]=H$} & \\
\hline $10_{\varepsilon}$ & $\mathfrak{p}$ & {$[H, \boldsymbol{B}]=-\varepsilon \boldsymbol{P}$} & {$[\boldsymbol{B}, \boldsymbol{B}]=\varepsilon \boldsymbol{J}$} & {$[\boldsymbol{B}, \boldsymbol{P}]=H$} & $\varepsilon= \pm 1$ \\
\hline 11 & $\mathfrak{s o}(D+1,1)$ & {$[H, \boldsymbol{B}]=\boldsymbol{B}$} & {$[H, \boldsymbol{P}]=-\boldsymbol{P}$} & {$[\boldsymbol{B}, \boldsymbol{P}]=H+\boldsymbol{J}$} & \\
\hline $12_{\varepsilon}$ & $\begin{array}{l}\mathfrak{s o}(D, 2) \\
\mathfrak{s o}(D+2)\end{array}$ & {$[H, \boldsymbol{B}]=-\varepsilon \boldsymbol{P}$} & {$[H, \boldsymbol{P}]=\varepsilon \boldsymbol{B}$} & {$[\boldsymbol{B}, \boldsymbol{P}]=H$} & $\varepsilon= \pm 1$ \\
\hline
\end{tabular}

Table 4. Generic kinematical Lie algebras for $D \geq 3$.

upon complexification) they are necessarily of the form

$$
(\boldsymbol{B}, \boldsymbol{P}, H) \mapsto(\boldsymbol{B}, \boldsymbol{P}, H)\left(\begin{array}{lll}
a & b & 0 \\
c & d & 0 \\
0 & 0 & \Delta
\end{array}\right)=(a \boldsymbol{B}+c \boldsymbol{P}, b \boldsymbol{B}+d \boldsymbol{P}, \Delta H),
$$

for some $a, b, c, d, \Delta \in \mathbb{R}$ and $\Delta(a d-b c) \neq 0$. In other words, the automorphism group of $\mathfrak{k}$ will be a subgroup of $\mathrm{GL}(2, \mathbb{R}) \times \mathbb{R}^{\neq 0}$.

\subsubsection{Lie pairs associated to Lie algebra \#1}

Kinematical Lie algebra \#1 in table 4 is isomorphic to the static Lie algebra, with all brackets zero except for those in equation (2.5). The automorphism group is the full $\operatorname{GL}(2, \mathbb{R}) \times \mathbb{R}^{\neq 0}$. An admissible Lie subalgebra is spanned by the rotations and the $D$ vectors $\alpha B_{a}+\beta P_{a}$, for some $\alpha, \beta \in \mathbb{R}$ not both zero. We will abbreviate the span of these vectors as $\alpha \boldsymbol{B}+\beta \boldsymbol{P}$, but let us not forget that we mean a $D$-dimensional subspace. Under an automorphism,

$$
\alpha \boldsymbol{B}+\beta \boldsymbol{P} \mapsto(a \alpha+b \beta) \boldsymbol{B}+(c \alpha+d \beta) \boldsymbol{P},
$$

so that all that happens is that the vector $(\alpha, \beta) \in \mathbb{R}^{2}$ is transformed under $\operatorname{GL}(2, \mathbb{R})$ according to the defining representation. Given any nonzero vector $(\alpha, \beta) \in \mathbb{R}^{2}$, there is a change of basis which sends it to the elementary vector $(1,0)$, so that up to the action of $\mathrm{GL}(2, \mathbb{R})$, we can take the Lie subalgebra $\mathfrak{h}$ to be spanned by the rotations and $\boldsymbol{B}$. 


\subsubsection{Lie pairs associated to Lie algebra \#2}

Kinematical Lie algebra \#2 in table 4 is isomorphic to the galilean Lie algebra. The automorphism group $A$ is easily determined to be

$$
A=\left\{\left(\begin{array}{ccc}
a & 0 & 0 \\
c & a \Delta & 0 \\
0 & 0 & \Delta
\end{array}\right) \mid a, c, \Delta \in \mathbb{R}, a \Delta \neq 0\right\} .
$$

Any vectorial subspace $W$ of the form $\alpha \boldsymbol{B}+\beta \boldsymbol{P}$ defines a subalgebra, since $\boldsymbol{B}$ and $\boldsymbol{P}$ commute. Under an automorphism,

$$
\left(\begin{array}{c}
\alpha \\
\beta
\end{array}\right) \mapsto\left(\begin{array}{c}
a \alpha \\
a \Delta \beta+c \alpha
\end{array}\right) .
$$

If $\alpha \neq 0$, we can choose $a=\alpha^{-1}$ and $c=-a^{2} \Delta \beta$ and hence bring $(\alpha, \beta) \mapsto(1,0)$, so that $W$ is the span of $\boldsymbol{B}$.

If $\alpha=0$, then $\beta \neq 0$ and we can choose $a \Delta=\beta^{-1}$ so that $(0, \beta) \mapsto(0,1)$. This means that now $W$ is the span of $\boldsymbol{P}$. We change basis in the Lie algebra so that $W$ is the span of $\boldsymbol{B}$. In the new basis, the galilean algebra has the additional bracket

$$
[H, \boldsymbol{P}]=\boldsymbol{B} .
$$

This is often known as the para-galilean algebra, but it is of course isomorphic to the galilean algebra.

\subsubsection{Lie pairs associated to Lie algebra $\# 3_{\gamma}$}

The automorphism group of Lie algebra $\# 3_{\gamma}$ in table 4 is determined to be

$$
A=\left\{\left(\begin{array}{lll}
a & 0 & 0 \\
0 & d & 0 \\
0 & 0 & 1
\end{array}\right) \quad a, d \in \mathbb{R}, a d \neq 0\right\} .
$$

Any vectorial subspace $W$ of the form $\alpha \boldsymbol{B}+\beta \boldsymbol{P}$ defines a subalgebra, since $\boldsymbol{B}$ and $\boldsymbol{P}$ commute. Under an automorphism,

$$
\left(\begin{array}{l}
\alpha \\
\beta
\end{array}\right) \mapsto\left(\begin{array}{c}
a \alpha \\
d \beta
\end{array}\right)
$$

We have three cases to consider, depending on whether $\alpha=0, \beta=0$ or neither are zero.

If $\beta=0$, then we can choose $a$ so that $a \alpha=1$, bringing $(\alpha, 0) \mapsto(1,0)$. Here $W$ is the span of $\boldsymbol{B}$.

If $\alpha=0$, then similarly $W$ is the span of $\boldsymbol{P}$. We change basis so that $W$ is again the span of $\boldsymbol{B}$, which means that the additional Lie brackets are now

$$
[H, \boldsymbol{B}]=\boldsymbol{B} \quad \text { and } \quad[H, \boldsymbol{P}]=\gamma \boldsymbol{P} .
$$

Finally, if $\alpha \beta \neq 0$, then we can choose $a, d$ so that $(\alpha, \beta) \mapsto(1,1)$ and $W$ is spanned by $\boldsymbol{B}+\boldsymbol{P}$. We change basis to that $W$ is spanned by $\boldsymbol{B}$, bringing the additional Lie brackets to the form

$$
[H, \boldsymbol{B}]=\boldsymbol{P} \quad \text { and } \quad[H, \boldsymbol{P}]=-\gamma \boldsymbol{B}+(1+\gamma) \boldsymbol{P} .
$$




\subsubsection{Lie pairs associated to Lie algebra \#4}

The automorphism group of Lie algebra \#4 in table 4 is determined to be

$$
A=\left\{\left(\begin{array}{lll}
a & b & 0 \\
c & d & 0 \\
0 & 0 & 1
\end{array}\right) \mid a, b, c, d \in \mathbb{R}, a d-b c \neq 0\right\} \cong \mathrm{GL}(2, \mathbb{R}) .
$$

Any vectorial subspace $\alpha \boldsymbol{B}+\beta \boldsymbol{P}$ is a subalgebra, but under the automorphisms we can always bring it to $\boldsymbol{B}$.

\subsubsection{Lie pairs associated to Lie algebra \#5}

The automorphism group of Lie algebra \#5 in table 4 is determined to be

$$
A=\left\{\left(\begin{array}{lll}
a & 0 & 0 \\
0 & d & 0 \\
0 & 0 & 1
\end{array}\right) \mid a, d \in \mathbb{R}, a d \neq 0\right\} \bigcup\left\{\left(\begin{array}{ccc}
0 & b & 0 \\
c & 0 & 0 \\
0 & 0 & -1
\end{array}\right) \mid b, c \in \mathbb{R}, b c \neq 0\right\} .
$$

Any vectorial subspace $\alpha \boldsymbol{B}+\beta \boldsymbol{P}$ is a subalgebra. The analysis of the action of automorphisms is very similar to that of algebra \#3, except that using automorphisms not in the identity component, we can relate the subspace spanned by $\boldsymbol{B}$ to that spanned by $\boldsymbol{P}$. Therefore we have two inequivalent Lie pairs, corresponding to the Lie algebra in the original basis:

$$
[H, \boldsymbol{B}]=-\boldsymbol{B} \quad \text { and } \quad[H, \boldsymbol{P}]=\boldsymbol{P},
$$

and the one corresponding to

$$
[H, \boldsymbol{B}]=\boldsymbol{P} \quad \text { and } \quad[H, \boldsymbol{P}]=\boldsymbol{B} .
$$

\subsubsection{Lie pairs associated to Lie algebra \#6}

The automorphism group of Lie algebra \#6 in table 4 is determined to be

$$
A=\left\{\left(\begin{array}{lll}
a & 0 & 0 \\
c & a & 0 \\
0 & 0 & 1
\end{array}\right) \quad a, c \in \mathbb{R}, a \neq 0\right\} .
$$

Any vectorial subspace $\alpha \boldsymbol{B}+\beta \boldsymbol{P}$ is a subalgebra. Using the automorphisms, we may send $(\alpha, \beta) \mapsto(a \alpha, c \alpha+a \beta)$. We can distinguish between two cases. If $\alpha \neq 0$, then take $a=\alpha^{-1}$ and $c=-\alpha^{-2} \beta$ so arrive at $(1,0)$, so that $W$ is spanned by $\boldsymbol{B}$. If $\alpha=0$, then $W$ is spanned by $\boldsymbol{P}$. In the former case, we have the original Lie brackets

$$
[H, \boldsymbol{B}]=\boldsymbol{B}+\boldsymbol{P} \quad \text { and } \quad[H, \boldsymbol{P}]=\boldsymbol{P} ;
$$

although we prefer to change basis so that $\boldsymbol{P} \mapsto-(\boldsymbol{P}+\boldsymbol{B})$. In that basis, the brackets are now

$$
[H, \boldsymbol{B}]=-\boldsymbol{P} \quad \text { and } \quad[H, \boldsymbol{P}]=B+2 \boldsymbol{P} .
$$

In the latter case, we change basis so that $W$ is again spanned by $\boldsymbol{B}$, but then the Lie brackets are now

$$
[H, \boldsymbol{B}]=\boldsymbol{B} \quad \text { and } \quad[H, \boldsymbol{P}]=\boldsymbol{B}+\boldsymbol{P} .
$$




\subsubsection{Lie pairs associated to Lie algebra $\# 7_{\chi}$}

The automorphism group of Lie algebra $\# 7_{\chi}$ in table 4 is determined to be

$$
A=\left\{\left(\begin{array}{ccc}
a & b & 0 \\
-b & a & 0 \\
0 & 0 & 1
\end{array}\right) \mid a, b \in \mathbb{R}, a^{2}+b^{2} \neq 0\right\} .
$$

Any vectorial subspace $\alpha \boldsymbol{B}+\beta \boldsymbol{P}$ is a subalgebra. Using the automorphisms we can bring any $(\alpha, \beta)$ to $(1,0)$, so that $W$ is spanned by $\boldsymbol{B}$. We prefer to change basis in the Lie algebra so that $\boldsymbol{P} \mapsto-(\boldsymbol{P}+\chi \boldsymbol{B})$. Doing so, the Lie brackets become

$$
[H, \boldsymbol{B}]=-\boldsymbol{P} \quad \text { and } \quad[H, \boldsymbol{P}]=\left(1+\chi^{2}\right) \boldsymbol{B}+2 \chi \boldsymbol{P} .
$$

\subsubsection{Lie pairs associated to Lie algebra \#8}

The automorphism group of Lie algebra \#8 in table 4 is determined to be

$$
\begin{aligned}
& A=\left\{\left(\begin{array}{ccc}
a & b & 0 \\
-b & a & 0 \\
0 & 0 & 1
\end{array}\right) \mid a, b \in \mathbb{R}, a^{2}+b^{2} \neq 0\right\} \\
& \bigcup\left\{\left(\begin{array}{ccc}
a & b & 0 \\
b & -a & 0 \\
0 & 0 & -1
\end{array}\right) \quad a, b \in \mathbb{R}, a^{2}+b^{2} \neq 0\right\} \text {. }
\end{aligned}
$$

Any vectorial subspace $\alpha \boldsymbol{B}+\beta \boldsymbol{P}$ is a subalgebra. As in the previous case, using only the automorphisms in the identity component we can already bring any $(\alpha, \beta)$ to $(1,0)$, so that $W$ is spanned by $\boldsymbol{B}$. There is thus a unique Lie pair associated to this Lie algebra.

\subsubsection{Lie pairs associated to Lie algebra \#9}

Lie algebra \#9 in table 4 is isomorphic to the Carroll algebra. Its automorphism group is determined to be

$$
A=\left\{\left(\begin{array}{ccc}
a & b & 0 \\
c & d & 0 \\
0 & 0 & a d-b c
\end{array}\right) \mid a, b, c, d \in \mathbb{R}, a d-b c \neq 0\right\} \cong \operatorname{GL}(2, \mathbb{R}) .
$$

Any vectorial subspace $\alpha \boldsymbol{B}+\beta \boldsymbol{P}$ is a subalgebra since

$$
\left[\alpha B_{a}+\beta P_{a}, \alpha B_{b}+\beta P_{b}\right]=\alpha \beta\left(\left[B_{a}, P_{b}\right]+\left[P_{a}, B_{b}\right]\right)=\alpha \beta\left(\delta_{a b} H-\delta_{b a} H\right)=0
$$

However up to the automorphisms we can always bring $(\alpha, \beta)$ to $(1,0)$ and hence $W$ is spanned by $\boldsymbol{B}$. There is a unique homogeneous Lie pair associated to the Carroll algebra. 


\subsubsection{Lie pairs associated to Lie algebra $\# 10_{\varepsilon}$}

Lie algebra $\# 10_{-1}$ is isomorphic to the euclidean Lie algebra, whereas $\# 10_{+1}$ is isomorphic to the Poincaré Lie algebra. The automorphism group is determined to be

$$
A=\left\{\left(\begin{array}{lll}
1 & 0 & 0 \\
c & d & 0 \\
0 & 0 & d
\end{array}\right) \mid c, d \in \mathbb{R}, d \neq 0\right\} \bigcup\left\{\left(\begin{array}{ccc}
-1 & 0 & 0 \\
c & d & 0 \\
0 & 0 & -d
\end{array}\right) \mid c, d \in \mathbb{R}, d \neq 0\right\} .
$$

Any vectorial subspace $\alpha \boldsymbol{B}+\beta \boldsymbol{P}$ is admissible, since

$$
[\alpha \boldsymbol{B}+\beta \boldsymbol{P}, \alpha \boldsymbol{B}+\beta \boldsymbol{P}]=\varepsilon \alpha^{2} \boldsymbol{J} .
$$

Under the automorphisms, we may bring $(\alpha, \beta)$ to $( \pm \alpha, c \alpha+d \beta)$. We must distinguish between two cases. If $\alpha \neq 0$, we can bring $(\alpha, \beta)$ to $(\alpha, 0)$ with $\alpha>0$, which says that $W$ is the span of $\boldsymbol{B}$. If $\alpha=0$, then $W$ is spanned by $\boldsymbol{P}$. In the former case, we have the original Lie brackets

$$
[H, \boldsymbol{B}]=-\varepsilon \boldsymbol{P} \quad[\boldsymbol{B}, \boldsymbol{P}]=H \quad \text { and } \quad[\boldsymbol{B}, \boldsymbol{B}]=\varepsilon \boldsymbol{J},
$$

whereas in the latter case we change basis so that $W$ is again spanned by $\boldsymbol{B}$, but this changes the Lie brackets to

$$
[H, \boldsymbol{P}]=\varepsilon \boldsymbol{B} \quad[\boldsymbol{B}, \boldsymbol{P}]=H \quad \text { and } \quad[\boldsymbol{P}, \boldsymbol{P}]=\varepsilon \boldsymbol{J},
$$

where we have also changed the sign of $H$ in order to keep the $[\boldsymbol{B}, \boldsymbol{P}]=H$ bracket uniform. These Lie algebras are often known as the para-Poincaré and para-euclidean algebras, depending on the sign of $\varepsilon$.

\subsubsection{Lie pairs associated to Lie algebra \#11}

Lie algebra \#11 in table 4 is isomorphic to $\mathfrak{s o}(D+1,1)$. The automorphism group consists of two connected components:

$$
A=\left\{\left(\begin{array}{ccc}
a & 0 & 0 \\
0 & a^{-1} & 0 \\
0 & 0 & 1
\end{array}\right) \mid a \in \mathbb{R}^{\neq 0}\right\} \bigcup\left\{\left(\begin{array}{ccc}
0 & b & 0 \\
b^{-1} & 0 & 0 \\
0 & 0 & -1
\end{array}\right) \mid b \in \mathbb{R}^{\neq 0}\right\} .
$$

Every vectorial subspace $\alpha \boldsymbol{B}+\beta \boldsymbol{P}$ is admissible, since

$$
[\alpha \boldsymbol{B}+\beta \boldsymbol{P}, \alpha \boldsymbol{B}+\beta \boldsymbol{P}]=2 \alpha \beta \boldsymbol{J} .
$$

If either $\alpha=0$ or $\beta=0$, then we can use the automorphisms to bring $(\alpha, \beta)$ to $(1,0)$, which says that $W$ is spanned by $\boldsymbol{B}$. If $\alpha \beta \neq 0$, then we can use the automorphisms to bring $(\alpha, \beta)$ to $(\sqrt{|\alpha \beta|}, \pm \sqrt{|\alpha \beta|})$, depending on whether $\alpha \beta$ is positive or negative. This says that $W$ is spanned by $\boldsymbol{B} \pm \boldsymbol{P}$. Changing basis so that $W$ is again spanned by $\boldsymbol{B}$, and redefining $H$, we arrive at the following brackets

$$
[H, \boldsymbol{B}]=\varepsilon \boldsymbol{P} \quad[H, \boldsymbol{P}]=\varepsilon \boldsymbol{B} \quad[\boldsymbol{B}, \boldsymbol{B}]=-\varepsilon \boldsymbol{J} \quad[\boldsymbol{P}, \boldsymbol{P}]=\varepsilon \boldsymbol{J} \quad \text { and } \quad[\boldsymbol{B}, \boldsymbol{P}]=H,
$$

where $\varepsilon= \pm 1$ according to whether $\pm \alpha \beta>0$. 


\subsubsection{Lie pairs associated to Lie algebra \#12 $\varepsilon_{\varepsilon}$}

Lie algebra \#12 $\varepsilon_{\varepsilon}$ in table 4 is isomorphic to $\mathfrak{s o}(D, 2)$ for $\varepsilon=1$ and to $\mathfrak{s o}(D+2)$ for $\varepsilon=-1$. The automorphism group is isomorphic to $O(2)$ and hence has two connected components:

$$
\begin{aligned}
A=\left\{\left(\begin{array}{ccc}
a & b & 0 \\
-b & a & 0 \\
0 & 0 & 1
\end{array}\right)\right. & \mid \begin{array}{c}
a, b \in \mathbb{R}, a^{2}+b^{2}=1 \\
A
\end{array} \\
& \bigcup\left\{\left(\begin{array}{ccc}
a & b & 0 \\
b & -a & 0 \\
0 & 0 & -1
\end{array}\right) \mid a, b \in \mathbb{R}, a^{2}+b^{2}=1\right\} .
\end{aligned}
$$

Any vectorial subspace $\alpha \boldsymbol{B}+\beta \boldsymbol{P}$ is admissible, since

$$
[\alpha \boldsymbol{B}+\beta \boldsymbol{P}, \alpha \boldsymbol{B}+\beta \boldsymbol{P}]=\varepsilon\left(\alpha^{2}+\beta^{2}\right) \boldsymbol{J} .
$$

However using only the automorphisms connected to the identity, we can rotate $(\alpha, \beta)$ to $\left(\sqrt{\alpha^{2}+\beta^{2}}, 0\right)$ and hence $W$ is spanned by $\boldsymbol{B}$. Therefore each of these kinematical Lie algebras has a unique Lie pair.

\subsubsection{Summary}

We summarise the above results in table 5 , which lists the equivalence classes of kinematical Lie pairs which exist for all $D \geq 3$. In this and other similar tables throughout the paper, each row consists of an incremental label "LP\#" for the Lie pair for easy reference in the rest of the paper and also a label "LA\#" of the isomorphism type of kinematical Lie algebra $\mathfrak{k}$ to which the Lie pair is associated. The rest of the row contains the Lie brackets of $\mathfrak{k}$ in a basis where the $\mathfrak{h}$ is spanned by $J_{a b}$ and $B_{a}$ and perhaps some relevant comments. In some cases we have made changes of basis (leaving alone the subalgebra $\mathfrak{h}$ ) in order to arrive at a more uniform description.

\subsection{Lie pairs unique to $D=3$}

Table 6 lists those kinematical Lie algebras which are unique to $D=3$. It is a sub-table of $\left[3\right.$, table 1], with small modifications. ${ }^{3}$ As usual we only list any nonzero Lie brackets in addition to $(2.5)$.

For the kinematical Lie algebras in this table, the condition on the vectorial subspace $W$ to be admissible is very restrictive and there is no need to worry about the action of the automorphisms. Therefore we have no need to determine the automorphism groups.

\subsubsection{Lie pairs associated to Lie algebra \#13 $\varepsilon_{\varepsilon}$}

The only linear combinations $\alpha \boldsymbol{B}+\beta \boldsymbol{P}$ which are admissible are those with $\beta=0$, so that $W$ is already spanned by $\boldsymbol{B}$. Therefore there is a unique spacetime for each of these kinematical Lie algebras.

\footnotetext{
${ }^{3}$ In [3, table 1] the rotational generator $\boldsymbol{R}$ is a vector and is related to $\boldsymbol{J}$ in table 6 by $J_{a b}=-\epsilon_{a b c} R_{c}$.
} 


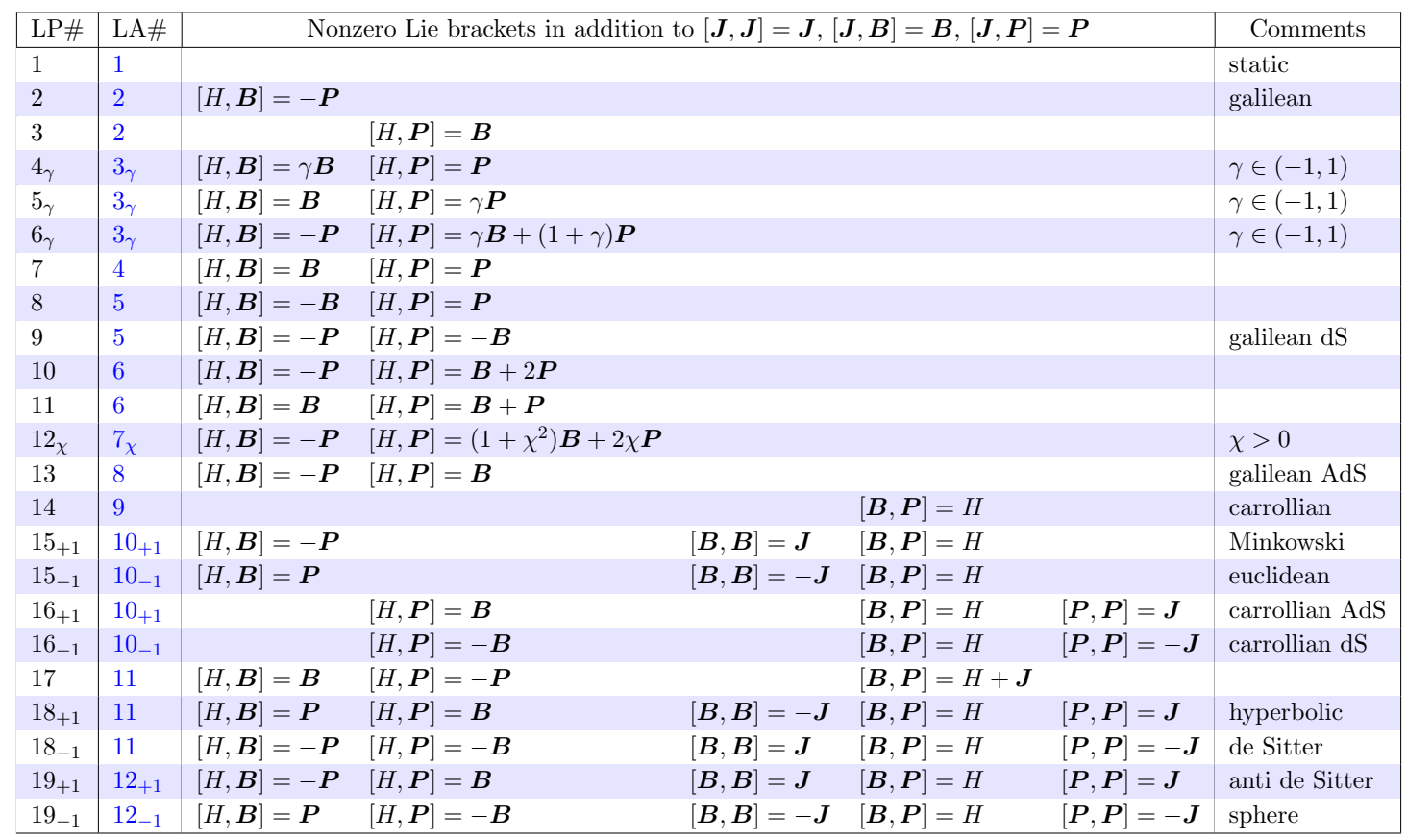

Table 5. Lie pairs for kinematical Lie algebras $(D \geq 3)$.

\begin{tabular}{|c|c|c|c|c|}
\hline LA\# & \multicolumn{3}{|c|}{ Nonzero brackets in addition to $[\boldsymbol{J}, \boldsymbol{J}]=\boldsymbol{J},[\boldsymbol{J}, \boldsymbol{B}]=\boldsymbol{B},[\boldsymbol{J}, \boldsymbol{P}]=\boldsymbol{P}$} & Comments \\
\hline $13_{\varepsilon}$ & & {$[\boldsymbol{B}, \boldsymbol{B}]=\boldsymbol{B}$} & {$[\boldsymbol{P}, \boldsymbol{P}]=\varepsilon(\boldsymbol{B}-\boldsymbol{J})$} & $\varepsilon= \pm 1$ \\
\hline 14 & & {$[\boldsymbol{B}, \boldsymbol{B}]=\boldsymbol{B}$} & & \\
\hline 15 & & {$[\boldsymbol{B}, \boldsymbol{B}]=\boldsymbol{P}$} & & \\
\hline 16 & {$[H, \boldsymbol{P}]=\boldsymbol{P}$} & {$[\boldsymbol{B}, \boldsymbol{B}]=\boldsymbol{B}$} & & \\
\hline 17 & {$[H, \boldsymbol{B}]=-\boldsymbol{P}$} & {$[\boldsymbol{B}, \boldsymbol{B}]=\boldsymbol{P}$} & & \\
\hline 18 & {$[H, B]=\boldsymbol{B}$} & {$[\boldsymbol{B}, \boldsymbol{B}]=\boldsymbol{P}$} & & \\
\hline
\end{tabular}

Table 6. Kinematical Lie algebras unique to $D=3$.

\subsubsection{Lie pairs associated to Lie algebra \#14}

There are two admissible subspaces: the span of $\boldsymbol{B}$ and the span of $\boldsymbol{P}$. In the first case, the Lie brackets are as shown in the table, whereas in the second case, changing basis so that $W$ is spanned by $\boldsymbol{B}$, we find

$$
[\boldsymbol{P}, \boldsymbol{P}]=\boldsymbol{P} \text {. }
$$

\subsubsection{Lie pairs associated to Lie algebra \#15}

The only admissible subspace is the one spanned by $\boldsymbol{P}$. Changing basis so that it spanned by $\boldsymbol{B}$, we arrive at the Lie bracket

$$
[\boldsymbol{P}, \boldsymbol{P}]=\boldsymbol{B} .
$$

\subsubsection{Lie pairs associated to Lie algebra \#16}

There are two admissible subspaces $W$ : the one spanned by $\boldsymbol{B}$ and the one spanned by $\boldsymbol{P}$. In the former case, the Lie brackets are as shown in the table, whereas in the latter case, 


\begin{tabular}{|l|l|rrr|l|}
\hline LP\# & LA\# & Nonzero Lie brackets in addition to $[\boldsymbol{J}, \boldsymbol{J}]=\boldsymbol{J},[\boldsymbol{J}, \boldsymbol{B}]=\boldsymbol{B},[\boldsymbol{J}, \boldsymbol{P}]=\boldsymbol{P}$ & Comments \\
\hline $20_{\varepsilon}$ & $13_{\varepsilon}$ & & {$[\boldsymbol{B}, \boldsymbol{B}]=\boldsymbol{B}$} & {$[\boldsymbol{P}, \boldsymbol{P}]=\varepsilon(\boldsymbol{B}-\boldsymbol{J})$} & $\varepsilon= \pm 1$ \\
21 & 14 & & {$[\boldsymbol{B}, \boldsymbol{B}]=\boldsymbol{B}$} & & \\
22 & 14 & & & {$[\boldsymbol{P}, \boldsymbol{P}]=\boldsymbol{P}$} & \\
23 & 15 & & & {$[\boldsymbol{P}, \boldsymbol{P}]=\boldsymbol{B}$} & \\
24 & 16 & & {$[H, \boldsymbol{P}]=\boldsymbol{P}$} & {$[\boldsymbol{B}, \boldsymbol{B}]=\boldsymbol{B}$} & \\
25 & 16 & {$[H, \boldsymbol{B}]=\boldsymbol{B}$} & & {$[\boldsymbol{P}, \boldsymbol{P}]=\boldsymbol{P}$} & \\
26 & 17 & & {$[H, \boldsymbol{P}]=-\boldsymbol{B}$} & {$[\boldsymbol{P}, \boldsymbol{P}]=\boldsymbol{B}$} & \\
27 & 18 & {$[H, \boldsymbol{B}]=2 \boldsymbol{B}$} & {$[H, \boldsymbol{P}]=\boldsymbol{P}$} & {$[\boldsymbol{P}, \boldsymbol{P}]=\boldsymbol{B}$} & \\
\hline
\end{tabular}

Table 7. Lie pairs for kinematical Lie algebras unique to $D=3$.

changing basis so that $W$ is spanned by $\boldsymbol{B}$ again, we arrive at

$$
[H, \boldsymbol{B}]=\boldsymbol{B} \quad \text { and } \quad[\boldsymbol{P}, \boldsymbol{P}]=\boldsymbol{P} .
$$

\subsubsection{Lie pairs associated to Lie algebra \#17}

There is a unique admissible subspace: the one spanned by $\boldsymbol{P}$. Changing basis so that it is spanned by $\boldsymbol{B}$, we arrive at the Lie bracket

$$
[H, \boldsymbol{P}]=-\boldsymbol{B} \quad \text { and } \quad[\boldsymbol{P}, \boldsymbol{P}]=\boldsymbol{B} .
$$

\subsubsection{Lie pairs associated to Lie algebra \#18}

The span of $\boldsymbol{P}$ is the only admissible subspace. Changing basis so that it is spanned by $\boldsymbol{B}$, we arrive at

$$
[H, \boldsymbol{B}]=2 \boldsymbol{B} \quad[H, \boldsymbol{P}]=\boldsymbol{P} \quad \text { and } \quad[\boldsymbol{P}, \boldsymbol{P}]=\boldsymbol{B} .
$$

\subsubsection{Summary}

Table 7 lists the Lie pairs associated to the kinematical Lie algebras which are unique to $D=3$.

\subsection{Lie pairs for $D=2$}

Table 8 lists the kinematical Lie algebras with $D=2$ in complex form. It is borrowed partially from [5, table 1$]$, where we explain the rationale for working with the complexified Lie algebras. If $\mathfrak{k}$ is a kinematical Lie algebra in $D=2$, we let its complexification $\mathfrak{k}_{\mathbb{C}}$ be the complex span of $H, J, \boldsymbol{B}, \boldsymbol{P}, \overline{\boldsymbol{B}}, \overline{\boldsymbol{P}}$, where $\boldsymbol{B}=B_{1}+i B_{2}, \boldsymbol{P}=P_{1}+i P_{2}, \overline{\boldsymbol{B}}=B_{1}-i B_{2}$ and $\overline{\boldsymbol{P}}=P_{1}-i P_{2}$. The standard rotational generator $J_{a b}$ is related to $J$ by $J_{a b}=-\epsilon_{a b} J$, from where we see that on a vectorial generator $\left[J, V_{a}\right]=\epsilon_{a b} V_{b}$, or equivalently $[J, \boldsymbol{V}]=-i \boldsymbol{V}$ in complex form. The Lie bracket of $\mathfrak{k}_{\mathbb{C}}$ is obtained from that of $\mathfrak{k}$ by extending it complex linearly. Given $\mathfrak{k}_{\mathbb{C}}$, we recover $\mathfrak{k}$ as the real Lie algebra which is fixed under the conjugation (i.e., complex antilinear involutive automorphism) $\star$ defined on generators by $H^{\star}=H$, $J^{\star}=J, \boldsymbol{B}^{\star}=\overline{\boldsymbol{B}}$ and $\boldsymbol{P}^{\star}=\overline{\boldsymbol{P}}$. To see how to translate between the complex and real notations, the bracket $[\boldsymbol{B}, \overline{\boldsymbol{B}}]=i H$ is equivalent to

$$
\left[B_{a}, B_{b}\right]=-\frac{1}{2} \epsilon_{a b} H,
$$




\begin{tabular}{|c|c|c|c|c|c|c|c|}
\hline LA\# & $\cong$ & \multicolumn{5}{|c|}{ Nonzero Lie brackets in addition to $[J, \boldsymbol{B}]=-i \boldsymbol{B},[J, \boldsymbol{P}]=-i \boldsymbol{P}$ and their c.c. } & \multirow[t]{2}{*}{ Comments } \\
\hline 19 & $\mathfrak{s}$ & & & & & & \\
\hline 20 & $\mathfrak{g}$ & {$[H, \boldsymbol{B}]=-\boldsymbol{P}$} & & & & & \\
\hline 21 & & {$[H, \boldsymbol{B}]=\boldsymbol{B}$} & {$[H, \boldsymbol{P}]=\boldsymbol{B}+\boldsymbol{I}$} & & & & \\
\hline $22_{\gamma+i \chi}$ & & {$[H, \boldsymbol{B}]=\boldsymbol{B}$} & {$[H, \boldsymbol{P}]=(\gamma+i$} & & & & $\gamma \in[-1,1], \chi \geq 0, \gamma+i \chi \neq-1$ \\
\hline 23 & $\mathfrak{n}_{-}$ & {$[H, \boldsymbol{B}]=\boldsymbol{B}$} & {$[H, P]=-\boldsymbol{P}$} & & & & \\
\hline 24 & $\mathfrak{n}_{+}$ & {$[H, \boldsymbol{B}]=i \boldsymbol{B}$} & & & & & \\
\hline 25 & c & & & & {$[\boldsymbol{B}, \overline{\boldsymbol{P}}]=H$} & & \\
\hline $26_{\varepsilon}$ & $\begin{array}{ll}p \\
\mathfrak{c}\end{array}$ & & {$[H, P]=\varepsilon \boldsymbol{B}$} & & {$[\boldsymbol{B}, \overline{\boldsymbol{P}}]=2 H$} & {$[\boldsymbol{P}, \overline{\boldsymbol{P}}]=\varepsilon 2 i J$} & $\varepsilon= \pm 1$ \\
\hline 27 & $\mathfrak{s o}(3,1)$ & {$[H, \boldsymbol{B}]=\boldsymbol{B}$} & {$[H, \boldsymbol{P}]=-\boldsymbol{P}$} & & {$[\boldsymbol{B}, \overline{\boldsymbol{P}}]=2(J$} & & \\
\hline $28_{\varepsilon}$ & $\begin{array}{l}\mathfrak{s o}(4) \\
\mathfrak{s o}(2,2)\end{array}$ & {$[H, \boldsymbol{B}]=\varepsilon \boldsymbol{P}$} & {$[H, \boldsymbol{P}]=-\varepsilon \boldsymbol{B}$} & {$[\boldsymbol{B}, \overline{\boldsymbol{B}}]=-\varepsilon 2 i J$} & {$[\boldsymbol{B}, \overline{\boldsymbol{P}}]=2 H$} & {$[\boldsymbol{P}, \overline{\boldsymbol{P}}]=-\varepsilon 2 i J$} & $\varepsilon= \pm 1$ \\
\hline 29 & & & & {$[\boldsymbol{B}, \overline{\boldsymbol{B}}]=i H$} & & {$[\boldsymbol{P}, \overline{\boldsymbol{P}}]=i H$} & \\
\hline 30 & & {$[H, \boldsymbol{B}]=i \boldsymbol{B}$} & & {$[\boldsymbol{B}, \overline{\boldsymbol{B}}]=i H$} & & {$[\boldsymbol{P}, \overline{\boldsymbol{P}}]=i(H+J)$} & \\
\hline 31 & & & & {$[\boldsymbol{B}, \overline{\boldsymbol{B}}]=i H$} & & & \\
\hline 32 & & {$[H, \boldsymbol{B}]=\boldsymbol{P}$} & & {$[\boldsymbol{B}, \overline{\boldsymbol{B}}]=i H$} & & & \\
\hline $33_{\varepsilon}$ & & {$[H, \boldsymbol{B}]=i \varepsilon \boldsymbol{B}$} & & {$[\boldsymbol{B}, \bar{B}]=i H$} & & & $\varepsilon= \pm 1$ \\
\hline
\end{tabular}

Table 8. Kinematical Lie algebras for $D=2$ (complex form).

whereas $[H, \boldsymbol{B}]=i \boldsymbol{B}$ is equivalent to

$$
\left[H, B_{a}\right]=-\epsilon_{a b} B_{b},
$$

et cetera. As usual, the Lie brackets (2.5), characterising kinematical Lie algebras, are implicit in every case.

Automorphisms are also different than for $D>2$ due to the fact that $\mathfrak{r}$ is onedimensional. If $\mathfrak{k}$ is a kinematical Lie algebra in table 8 , then the group of automorphisms is a subgroup of $\mathrm{GL}(2, \mathbb{C}) \times \mathrm{GL}(2, \mathbb{R})$. It consists of pairs

$$
\left(\begin{array}{ll}
a & b \\
c & d
\end{array}\right) \in \mathrm{GL}(2, \mathbb{C}) \quad \text { and } \quad\left(\begin{array}{ll}
r & t \\
s & 1
\end{array}\right) \in \mathrm{GL}(2, \mathbb{R})
$$

acting on the basis by

$$
(\boldsymbol{B}, \boldsymbol{P}) \mapsto(a \boldsymbol{B}+c \boldsymbol{P}, b \boldsymbol{B}+d \boldsymbol{P}) \quad \text { and } \quad(H, J) \mapsto(r H+s J, J+t H) .
$$

(This uses the observation that for any of the Lie algebras in table 8, the only elements in the real span of $H$ and $J$ which act on $\boldsymbol{B}$ and $\boldsymbol{P}$ as rotations have the form $J+t H$ and $t$ can be nonzero only when $H$ is central, which is the case for the static and Carroll algebras and also for the kinematical Lie algebras \#29 and \#31 in table 8.)

If $\mathfrak{h}$ is an admissible subalgebra, its complexification $\mathfrak{h}_{\mathbb{C}}$ is the complex span of $\boldsymbol{J}+$ $t H, \alpha \boldsymbol{B}+\beta \boldsymbol{P}, \bar{\alpha} \overline{\boldsymbol{B}}+\bar{\beta} \overline{\boldsymbol{P}}$, for some $\alpha, \beta \in \mathbb{C}$ not both zero and some $t \in \mathbb{R}$. (Again this uses the above mentioned observation that the rotational generators are of the form $\boldsymbol{J}+t H$.) The real subalgebra $\mathfrak{h}$ is then the real span of $\boldsymbol{J}+t H$ and the real and imaginary parts of $\alpha \boldsymbol{B}+\beta \boldsymbol{P}$. We let $W$ denote the two-dimensional real vector space spanned by the real and imaginary parts of $\alpha \boldsymbol{B}+\beta \boldsymbol{P}$, which transforms as a two-dimensional real (vector) representation of $\mathfrak{r}$. We will, however, abbreviate this by saying $W$ is spanned by $\alpha \boldsymbol{B}+\beta \boldsymbol{P}$. 


\subsubsection{Lie pairs associated to Lie algebra \#19}

Any subspace $\alpha \boldsymbol{B}+\beta \boldsymbol{P}$ is admissible. The automorphism group is the full group

$$
A=\left\{\left(\begin{array}{rrr}
a & b & \\
c & d & \\
& r & t \\
& s & 1
\end{array}\right) \mid a, b, c, d \in \mathbb{C}, r, s, t \in \mathbb{R}, r(a d-b c) \neq 0\right\} .
$$

As in the case of $D \geq 3$, we may bring $(\alpha, \beta)$ to $(1,0)$ by an automorphism which preserves $H$ and $J$, so we can take $W$ to be spanned by $\boldsymbol{B}$ without loss of generality. There is thus a unique spacetime associated to this Lie algebra.

\subsubsection{Lie pairs associated to the Lie algebra \#20}

Any subspace $\alpha \boldsymbol{B}+\beta \boldsymbol{P}$ is admissible. The automorphism group is now

$$
A=\left\{\left(\begin{array}{ccc}
a & 0 & \\
c & a r & \\
& r & 0 \\
& 0 & 1
\end{array}\right) \mid a, c \in \mathbb{C}, r \in \mathbb{R}, \text { ar } \neq 0\right\} .
$$

Using the automorphisms, we can bring any nonzero $(\alpha, \beta) \in \mathbb{C}^{2}$ to either $(1,0)$ or $(0,1)$, depending on whether or not $\alpha=0$. In the former case, $W$ is spanned by $\boldsymbol{B}$ and the nonzero brackets are unchanged. In the latter case, $W$ is spanned by $\boldsymbol{P}$. Changing basis so that it is again spanned by $\boldsymbol{B}$ changes the nonzero brackets to $[H, \boldsymbol{P}]=-\boldsymbol{B}$.

\subsubsection{Lie pairs associated to Lie algebra \#21}

Again every nonzero subspace $\alpha \boldsymbol{B}+\beta \boldsymbol{P}$ is admissible. The automorphisms are given by

$$
A=\left\{\left(\begin{array}{ccc}
a & b & \\
0 & a & \\
& 1 & 0 \\
& 0 & 1
\end{array}\right) \mid a, b \in \mathbb{C}, a \neq 0\right\} .
$$

Using automorphisms we can bring any nonzero $(\alpha, \beta)$ to one of two vectors: $(1,0)$ or $(0,1)$, depending on whether or not $\beta=0$. In the former case, $W$ is already spanned by $\boldsymbol{B}$ and the nonzero brackets are unchanged. In the latter case, $W$ is spanned by $\boldsymbol{P}$, but changing basis so that it is again spanned by $\boldsymbol{B}$, results in the following nonzero Lie brackets:

$$
[H, \boldsymbol{B}]=\boldsymbol{B}+\boldsymbol{P} \quad \text { and } \quad[H, \boldsymbol{P}]=\boldsymbol{P} .
$$

\subsubsection{Lie pairs associated to Lie algebras \#22 ${ }_{\gamma+i \chi}$ and \#23}

These two cases can be treated simultaneously, with brackets

$$
[H, \boldsymbol{B}]=\boldsymbol{B} \quad \text { and } \quad[H, \boldsymbol{P}]=\xi \boldsymbol{P},
$$

for $\xi \in \mathbb{C}$ given by $\xi=\gamma+i \chi$ with $\gamma \in[-1,1]$ and $\chi \geq 0$. For kinematical Lie algebra $\# 22_{\gamma+i \chi}, \xi \neq-1$, which corresponds to Lie algebra \#23. It is convenient to distinguish three cases: 
1. $\xi=1$

2. $\operatorname{Re} \xi=-1 ;$ and

3. $\xi \neq 1$ and $\operatorname{Re} \xi \neq-1$.

In all cases, any nonzero subspace $\alpha \boldsymbol{B}+\beta \boldsymbol{P}$ is admissible, but the automorphisms differ.

For $\xi=1$, the automorphisms are given by

$$
A=\left\{\left(\begin{array}{lll}
a & b & \\
c & d & \\
& 1 & 0 \\
& 0 & 1
\end{array}\right) \mid a, b, c, d \in \mathbb{C}, a d-b c \neq 0\right\},
$$

and it is clear that we can bring any nonzero $(\alpha, \beta) \in \mathbb{C}^{2}$ to $(1,0)$. So without loss of generality we can take $W$ to be spanned by $\boldsymbol{B}$ and the brackets are unchanged:

$$
[H, \boldsymbol{B}]=\boldsymbol{B} \quad \text { and } \quad[H, \boldsymbol{P}]=\boldsymbol{P} .
$$

For $\operatorname{Re} \xi=-1$, the automorphisms are given by

$$
A=\left\{\left(\begin{array}{lll}
a & 0 & \\
0 & d & \\
& 1 & 0 \\
& 0 & 1
\end{array}\right) \quad a, d \in \mathbb{C}, a d \neq 0\right\} \bigcup\left\{\left(\begin{array}{rrr}
0 & b & \\
c & 0 & \\
& -1 & 0 \\
& -\chi & 1
\end{array}\right) \mid b, c \in \mathbb{C}, b c \neq 0\right\} .
$$

Any nonzero $(\alpha, \beta) \in \mathbb{C}^{2}$ may be brought to one of two normal forms: $(1,0)$ or $(1,1)$ according to whether one of $\alpha$ or $\beta$ are zero or neither are zero, respectively. In the first case, $W$ is already spanned by $\boldsymbol{B}$ and there is no need to change basis. In the second case, $W$ is spanned by $\boldsymbol{B}+\boldsymbol{P}$ and after the change of basis, the new brackets are

$$
[H, \boldsymbol{B}]=\boldsymbol{P} \quad \text { and } \quad[H, \boldsymbol{P}]=-\xi \boldsymbol{B}+(1+\xi) \boldsymbol{P} .
$$

Finally, if $\xi \neq 1$ and $\operatorname{Re} \xi \neq-1$, the automorphisms are

$$
A=\left\{\left(\begin{array}{rrr}
a & 0 & \\
0 & d & \\
& 1 & 0 \\
& 0 & 1
\end{array}\right) \mid a, d \in \mathbb{C}, a d \neq 0\right\} .
$$

Again we can bring any nonzero $(\alpha, \beta)$ to one of three normal forms $(1,0)$ (if $\beta=0),(0,1)$ (if $\alpha=0$ ) or $(1,1)$ otherwise. In the first case there is no need to change basis. In the second case, $W$ is spanned by $\boldsymbol{P}$ and changing basis results in

$$
[H, \boldsymbol{B}]=\xi B \quad \text { and } \quad[H, \boldsymbol{P}]=\boldsymbol{P} .
$$

Finally, in the last case, $W$ is spanned by $\boldsymbol{B}+\boldsymbol{P}$. Changing basis results again in

$$
[H, \boldsymbol{B}]=\boldsymbol{P} \quad \text { and } \quad[H, \boldsymbol{P}]=-\xi \boldsymbol{B}+(1+\xi) \boldsymbol{P} .
$$




\subsubsection{Lie pairs associated to Lie algebra \#24}

Again every nonzero subspace $W$ is admissible. The automorphism group has now two connected components:

$$
A=\left\{\left(\begin{array}{rrr}
a & 0 & \\
0 & d & \\
& 1 & 0 \\
& 0 & 1
\end{array}\right) \quad a, d \in \mathbb{C}, a d \neq 0\right\} \bigcup\left\{\left(\begin{array}{rrr}
0 & b & \\
c & 0 & \\
& -1 & 0 \\
-1 & 1
\end{array}\right) \mid b, c \in \mathbb{C}, b c \neq 0\right\} .
$$

Via an automorphism, any nonzero $(\alpha, \beta) \in \mathbb{C}^{2}$ can be brought to either $(1,0)$ if at least one of $\alpha$ or $\beta$ is zero, or $(1,1)$ otherwise. In the former case, $W$ is already spanned by $\boldsymbol{B}$ and the brackets are unchanged, whereas in the latter case $W$ is spanned by $\boldsymbol{B}+\boldsymbol{P}$ and changing basis changes the brackets to

$$
[H, \boldsymbol{B}]=-\boldsymbol{P} \quad \text { and } \quad[H, \boldsymbol{P}]=\boldsymbol{B} .
$$

\subsubsection{Lie pairs associated to Lie algebra \#25}

A subspace $\alpha \boldsymbol{B}+\beta \boldsymbol{P}$ is admissible if and only if $\alpha \bar{\beta} \in \mathbb{R}$ : indeed,

$$
[\alpha \boldsymbol{B}+\beta \boldsymbol{P}, \bar{\alpha} \overline{\boldsymbol{B}}+\bar{\beta} \overline{\boldsymbol{P}}]=(\alpha \bar{\beta}-\bar{\alpha} \beta) H .
$$

The condition $\alpha \bar{\beta} \in \mathbb{R}$ means that we can choose $\alpha$ and $\beta$ real and still span the same subspace. It is unnecessary to determine the precise automorphism group. It suffices to remark that it includes the subgroup

$$
A=\left\{\left(\begin{array}{ccc}
a & b & \\
c & d & \\
& a d-b c & 0 \\
& 0 & 1
\end{array}\right) \quad a, b, c, d \in \mathbb{R}, a d-b c \neq 0\right\},
$$

and that using such an automorphism, we can take any $(\alpha, \beta) \in \mathbb{R}^{2}$ to $(1,0)$. There is thus a unique spacetime.

\subsubsection{Lie pairs associated to Lie algebra \#26 $\varepsilon_{\varepsilon}$}

A subspace $\alpha \boldsymbol{B}+\beta \boldsymbol{P}$ is admissible if and only if $\alpha \bar{\beta}$ is real:

$$
[\alpha \boldsymbol{B}+\beta \boldsymbol{P}, \bar{\alpha} \overline{\boldsymbol{B}}+\bar{\beta} \overline{\boldsymbol{P}}]=2(\alpha \bar{\beta}-\bar{\alpha} \beta) H+2 i \varepsilon|\beta|^{2} J .
$$

As in the previous case, we can take $\alpha, \beta$ both real without altering their span.

The automorphism group consists of

$$
A=\left\{\left(\begin{array}{ccc}
r d & u d & \\
0 & d & \\
& r & 0 \\
& 0 & 1
\end{array}\right)|r, u \in \mathbb{R}, d \in \mathbb{C}, r \neq 0,| d \mid=1\right\} .
$$

Under automorphisms we can bring every admissible subspace to either the span of the $\boldsymbol{B}$ or the span of the $\boldsymbol{P}$. In the former case we do not change the brackets, whereas in the latter case we change basis and arrive at

$$
[H, \boldsymbol{B}]=\varepsilon \boldsymbol{P} \quad[\boldsymbol{B}, \overline{\boldsymbol{P}}]=-2 H \quad \text { and } \quad[\boldsymbol{B}, \overline{\boldsymbol{B}}]=2 \varepsilon i J .
$$




\subsubsection{Lie pairs associated to Lie algebra \#27}

Because of the bracket

$$
[\alpha \boldsymbol{B}+\beta \boldsymbol{P}, \bar{\alpha} \overline{\boldsymbol{B}}+\bar{\beta} \overline{\boldsymbol{P}}]=2(\alpha \bar{\beta}-\bar{\alpha} \beta) J-2 i(\alpha \bar{\beta}+\bar{\alpha} \beta) H,
$$

a subspace $\alpha \boldsymbol{B}+\beta \boldsymbol{P}$ is admissible if and only if $\alpha \bar{\beta}+\bar{\alpha} \beta=0$.

The automorphism group in this case can be determined to consist of two connected components

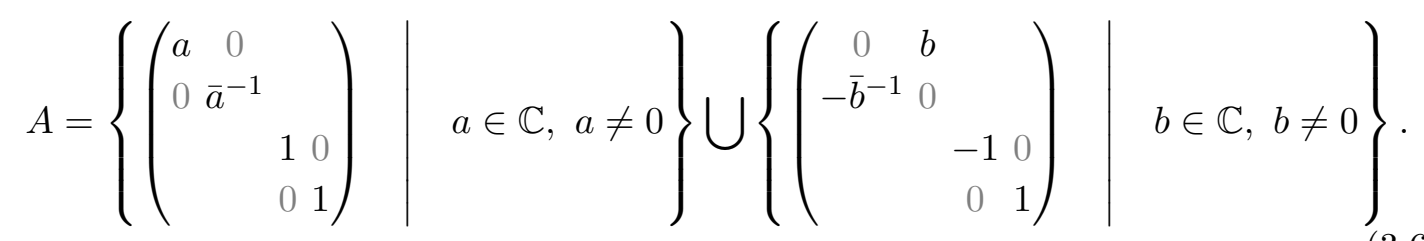

Under an automorphism in the identity component, $(\alpha, \beta) \mapsto(a \alpha, \beta / \bar{a})$, whereas under an automorphism in the other component $(\alpha, \beta) \mapsto(b \beta,-\alpha / \bar{b})$. Notice that the product $\alpha \bar{\beta}$ remains invariant under automorphisms, provided that $(\alpha, \beta)$ is admissible, so that $\alpha \bar{\beta}=-\bar{\alpha} \beta$.

Suppose that $\alpha \bar{\beta}=0$. Then either $\alpha=0$ or $\beta=0$ and we may apply an automorphism to send such $(\alpha, \beta)$ to $(1,0)$. On the other hand, if $\alpha \bar{\beta}=i t$ for some real $t \neq 0$, we can always choose an automorphism to bring $(\alpha, \beta)$ to a multiple of $(1, \pm i)$. The former case, $W$ is spanned by $\boldsymbol{B}$ and in the latter cases by $\boldsymbol{B} \pm i \boldsymbol{P}$. In these latter cases, changing basis so that $W$ is spanned again by $\boldsymbol{B}$, we find the following brackets:

$$
[H, \boldsymbol{B}]=\boldsymbol{P} \quad[H, \boldsymbol{P}]=\boldsymbol{B} \quad[\boldsymbol{B}, \overline{\boldsymbol{B}}]=\mp i J \quad[\boldsymbol{P}, \overline{\boldsymbol{P}}]= \pm i J \quad \text { and } \quad[\boldsymbol{B}, \overline{\boldsymbol{P}}]= \pm H .
$$

\subsubsection{Lie pairs associated to Lie algebra $\# 28_{\varepsilon}$}

A subspace $\alpha \boldsymbol{B}+\beta \boldsymbol{P}$ is admissible if and only if $\alpha \bar{\beta} \in \mathbb{R}$. Such an admissible subspace is therefore always of the form $\alpha \boldsymbol{B}+\beta \boldsymbol{P}$ with $\alpha, \beta \in \mathbb{R}$. It is not necessary to determine the precise form of the automorphism group, but simply to remark that it contains the following $\mathrm{SO}(2)$ subgroup:

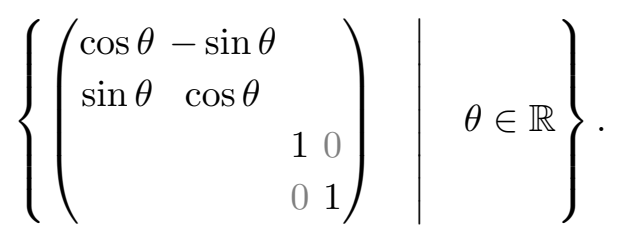

Any $(\alpha, \beta) \in \mathbb{R}^{2}$ can be rotated to $\left(\sqrt{\alpha^{2}+\beta^{2}}, 0\right)$ which corresponds to the subspace spanned by $\boldsymbol{B}$. Therefore there is a unique spacetime for each sign $\varepsilon$.

\subsubsection{No Lie pairs associated to Lie algebras \#29 and \#30}

There is no admissible subalgebra in these cases, since in one case

$$
[\alpha \boldsymbol{B}+\beta \boldsymbol{P}, \bar{\alpha} \overline{\boldsymbol{B}}+\bar{\beta} \overline{\boldsymbol{P}}]=i\left(|\alpha|^{2}+|\beta|^{2}\right) H
$$


and in the other

$$
[\alpha \boldsymbol{B}+\beta \boldsymbol{P}, \bar{\alpha} \overline{\boldsymbol{B}}+\bar{\beta} \overline{\boldsymbol{P}}]=i\left(|\alpha|^{2}+|\beta|^{2}\right) H+i|\beta|^{2} J,
$$

whereas the right-hand sides do not span a rotational subalgebra. Therefore there are no spacetimes associated these kinematical Lie algebras.

\subsubsection{Lie pairs associated to Lie algebras \#31, \#32 and \#33}

We can treat these cases simultaneously, since they only differ in the adjoint action of $H$. In all cases, the only admissible subspace is the one spanned by $\boldsymbol{P}$, since

$$
[\alpha \boldsymbol{B}+\beta \boldsymbol{P}, \bar{\alpha} \overline{\boldsymbol{B}}+\bar{\beta} \overline{\boldsymbol{P}}]=i|\alpha|^{2} H,
$$

and $H$ does not span a rotational subalgebra. Changing basis so that it is spanned by $\boldsymbol{B}$, changes the brackets to

$$
[H, \boldsymbol{P}]=\left\{\begin{array}{l}
0 \\
\boldsymbol{B} \\
i \varepsilon \boldsymbol{P}
\end{array} \quad \text { and } \quad[\boldsymbol{P}, \overline{\boldsymbol{P}}]=i H .\right.
$$

\subsubsection{Summary}

In table 9, we list the Lie pairs associated to the kinematical Lie algebras in $D=2$. We have reverted to the real form of the $D=2$ Lie algebras. The notation is then very similar to $D \geq 3$. In particular, the rotational generator $\boldsymbol{J}$ is again $J_{a b}$ as in $D \geq 3$. This eases the comparison with $D \geq 3$ and allows us to quickly determine which Lie pairs are unique to $D=2$ and which are just the $D=2$ avatars of Lie pairs which exist also for $D>2$. The main difference between $D=2$ and $D>2$ is that some brackets feature the $\epsilon$ symbol and we have therefore introduced $\widetilde{\boldsymbol{P}}$ and $\widetilde{\boldsymbol{B}}$ as explained at the start of section 2.2 . We have also changed basis in some cases in order to uniformise the presentation and ease the comparison with $D>2$.

The table is divided into two: the ones above the horizontal line are $D=2$ avatars of Lie pairs which exist also for $D>2$. The precise dictionary is shown in table 10 . The ones below the line exist only for $D=2$.

\subsection{Lie pairs for $D=1$}

Finally, we consider the Lie pairs associated to the $D=1$ kinematical Lie algebras. Since there are no rotations in $D=1$, every three-dimensional real Lie algebra is kinematical. Such Lie algebras were classified by Bianchi [48] as part of his classification of threedimensional homogeneous manifolds. In this section, however, they will be associated to two-dimensional homogeneous spaces. The Bianchi classification of three-dimensional real Lie algebras is recalled in table 11. We have omitted Bianchi III because it is isomorphic to Bianchi $\mathrm{VI}_{1}$.

Because of dimension and the absence of rotations, every one-dimensional subspace is an admissible subalgebra. Therefore to classify pairs $(\mathfrak{k}, \mathfrak{h})$ we need, for each $\mathfrak{k}$ in the Bianchi 


\begin{tabular}{|c|c|c|c|c|c|c|c|}
\hline$-\mathrm{LP} \#$ & LA\# & & Nonzero Lie brackets in addition to $[J$ & $\boldsymbol{T}, \boldsymbol{B}]=\boldsymbol{B}$ and & $\boldsymbol{J}, \boldsymbol{P}]=\boldsymbol{P}$ & & Comments \\
\hline 28 & 19 & & & & & & static \\
\hline 29 & 20 & {$[H, \boldsymbol{B}]=-\boldsymbol{P}$} & & & & & galilean \\
\hline 30 & 20 & & {$[H, \boldsymbol{P}]=\boldsymbol{B}$} & & & & \\
\hline 31 & 21 & {$[H, \boldsymbol{B}]=-\boldsymbol{P}$} & {$[H, \boldsymbol{P}]=\boldsymbol{B}+2 \boldsymbol{P}$} & & & & \\
\hline 32 & 21 & {$[H, \boldsymbol{B}]=\boldsymbol{B}$} & {$[H, \boldsymbol{P}]=\boldsymbol{B}+\boldsymbol{P}$} & & & & \\
\hline 33 & $22_{1+i 0}$ & {$[H, \boldsymbol{B}]=\boldsymbol{B}$} & {$[H, P]=\boldsymbol{P}$} & & & & \\
\hline 34 & 23 & {$[H, \boldsymbol{B}]=-\boldsymbol{B}$} & {$[H, \boldsymbol{P}]=\boldsymbol{P}$} & & & & \\
\hline 35 & 23 & {$[H, \boldsymbol{B}]=-\boldsymbol{P}$} & {$[H, P]=-\boldsymbol{B}$} & & & & galilean dS \\
\hline $36_{\gamma}$ & $22_{\gamma+i 0}$ & {$[H, \boldsymbol{B}]=\boldsymbol{B}$} & {$[H, \boldsymbol{P}]=\gamma \boldsymbol{P}$} & & & & $-1<\gamma<1$ \\
\hline $37_{\gamma}$ & $22_{\gamma+i 0}$ & {$[H, \boldsymbol{B}]=\gamma \boldsymbol{B}$} & {$[H, P]=\boldsymbol{P}$} & & & & $-1<\gamma<1$ \\
\hline $38_{\gamma}$ & $22_{\gamma+i 0}$ & {$[H, \boldsymbol{B}]=-\boldsymbol{P}$} & {$[H, \boldsymbol{P}]=\gamma \boldsymbol{B}+(1+\gamma) \boldsymbol{P}$} & & & & $-1<\gamma<1$ \\
\hline 39 & 24 & {$[H, \boldsymbol{B}]=-\boldsymbol{P}$} & {$[H, P]=\boldsymbol{B}$} & & & & galilean AdS \\
\hline 40 & 25 & & & & {$[\boldsymbol{B}, \boldsymbol{P}]=H$} & & carrollian \\
\hline 41 & $26+1$ & & {$[H, P]=\boldsymbol{B}$} & & {$[\boldsymbol{B}, \boldsymbol{P}]=H$} & {$[P, P]=J$} & carrollian AdS \\
\hline 42 & $26_{-1}$ & & {$[H, \boldsymbol{P}]=-\boldsymbol{B}$} & & {$[\boldsymbol{B}, \boldsymbol{P}]=H$} & {$[\boldsymbol{P}, \boldsymbol{P}]=-\boldsymbol{J}$} & carrollian dS \\
\hline 43 & $26_{+1}$ & {$[H, \boldsymbol{B}]=-\boldsymbol{P}$} & & {$[\boldsymbol{B}, \boldsymbol{B}]=\boldsymbol{J}$} & {$[\boldsymbol{B}, \boldsymbol{P}]=H$} & & Minkowski \\
\hline 44 & $26_{-1}$ & {$[H, \boldsymbol{B}]=\boldsymbol{P}$} & & {$[\boldsymbol{B}, \boldsymbol{B}]=-\boldsymbol{J}$} & {$[\boldsymbol{B}, \boldsymbol{P}]=H$} & & euclidean \\
\hline 45 & 27 & {$[H, \boldsymbol{B}]=\boldsymbol{B}$} & {$[H, P]=-\boldsymbol{P}$} & & {$[\boldsymbol{B}, \boldsymbol{P}]=H+\boldsymbol{J}$} & & \\
\hline 46 & 27 & {$[H, \boldsymbol{B}]=\boldsymbol{P}$} & {$[H, \boldsymbol{P}]=\boldsymbol{B}$} & {$[\boldsymbol{B}, \boldsymbol{B}]=-\boldsymbol{J}$} & {$[\boldsymbol{B}, \boldsymbol{P}]=H$} & {$[\boldsymbol{P}, \boldsymbol{P}]=\boldsymbol{J}$} & hyperbolic \\
\hline 47 & 27 & {$[H, \boldsymbol{B}]=-\boldsymbol{P}$} & {$[H, P]=-\boldsymbol{B}$} & {$[\boldsymbol{B}, \boldsymbol{B}]=\boldsymbol{J}$} & {$[\boldsymbol{B}, \boldsymbol{P}]=H$} & {$[\boldsymbol{P}, \boldsymbol{P}]=-\boldsymbol{J}$} & de Sitter \\
\hline 48 & $28+1$ & {$[H, \boldsymbol{B}]=\boldsymbol{P}$} & {$[H, \boldsymbol{P}]=-\boldsymbol{B}$} & {$[\boldsymbol{B}, \boldsymbol{B}]=-\boldsymbol{J}$} & {$[\boldsymbol{B}, \boldsymbol{P}]=H$} & {$[\boldsymbol{P}, \boldsymbol{P}]=-\boldsymbol{J}$} & sphere \\
\hline 49 & $28_{-1}$ & {$[H, \boldsymbol{B}]=-\boldsymbol{P}$} & {$[H, P]=\boldsymbol{B}$} & {$[\boldsymbol{B}, \boldsymbol{B}]=\boldsymbol{J}$} & {$[\boldsymbol{B}, \boldsymbol{P}]=H$} & {$[\boldsymbol{P}, \boldsymbol{P}]=\boldsymbol{J}$} & anti de Sitter \\
\hline $50 \chi$ & $22_{1+i \chi}$ & {$[H, \boldsymbol{B}]=-\boldsymbol{P}$} & {$[H, \boldsymbol{P}]=\left(1+4 \chi^{-2}\right) \boldsymbol{B}+4 \chi^{-1} \boldsymbol{P}$} & & & & $\chi>0$ \\
\hline $51_{\chi}$ & $22_{1+i \chi}$ & {$[H, \boldsymbol{B}]=\boldsymbol{B}-\chi \widetilde{\boldsymbol{B}}$} & {$[H, \boldsymbol{P}]=\boldsymbol{P}$} & & & & $\chi>0$ \\
\hline $522_{x}$ & $22_{1+i \chi}$ & {$[H, \boldsymbol{B}]=\boldsymbol{B}$} & {$[H, \boldsymbol{P}]=\boldsymbol{P}-\chi \widetilde{\boldsymbol{P}}$} & & & & $\chi>0$ \\
\hline $53_{\gamma, \chi}$ & $22_{\gamma+i \chi}$ & {$[H, \boldsymbol{B}]=\boldsymbol{B}$} & {$[H, \boldsymbol{P}]=\gamma \boldsymbol{P}-\chi \widetilde{\boldsymbol{P}}$} & & & & $-1 \leq \gamma<1, \chi>0$ \\
\hline $54_{\gamma, \chi}$ & $22_{\gamma+i \chi}$ & {$[H, \boldsymbol{B}]=\gamma \boldsymbol{B}-\chi \widetilde{\boldsymbol{B}}$} & {$[H, P]=\boldsymbol{P}$} & & & & $-1 \leq \gamma<1, \chi>0$ \\
\hline $55_{\gamma, \chi}$ & $22_{\gamma+i \chi}$ & {$[H, \boldsymbol{B}]=-\underset{\sim}{\boldsymbol{P}}$} & {$[H, \boldsymbol{P}]=(1+\gamma) \boldsymbol{P}-\chi \widetilde{\boldsymbol{P}}+\gamma \boldsymbol{B}-\chi \widetilde{\boldsymbol{B}}$} & & & & $-1 \leq \gamma<1, \chi>0$ \\
\hline 56 & 24 & {$[H, \boldsymbol{B}]=-\widetilde{\boldsymbol{B}}$} & & & & & \\
\hline 57 & 31 & & & & & {$[\boldsymbol{P}, \boldsymbol{P}]=H$} & \\
\hline 58 & 32 & & {$[H, \boldsymbol{P}]=\boldsymbol{B}$} & & & {$[\boldsymbol{P}, \boldsymbol{P}]=-H$} & \\
\hline 59 & $33_{+1}$ & & {$[H, \boldsymbol{P}]=\widetilde{\boldsymbol{P}}$} & & & {$[\boldsymbol{P}, \boldsymbol{P}]=H$} & \\
\hline 60 & $33_{-1}$ & & {$[H, \boldsymbol{P}]=\widetilde{\boldsymbol{P}}$} & & & {$[\boldsymbol{P}, \boldsymbol{P}]=-H$} & \\
\hline
\end{tabular}

Table 9. Lie pairs for kinematical Lie algebras $(D=2$, real form).

\begin{tabular}{|l|l|}
\hline$D=2$ & $D \geq 3$ \\
\hline 28 & 1 \\
29 & 2 \\
30 & 3 \\
31 & 10 \\
32 & 11 \\
33 & 7 \\
34 & 8 \\
35 & 9 \\
\hline
\end{tabular}

\begin{tabular}{|l|l|}
\hline$D=2$ & $D \geq 3$ \\
\hline $36_{\gamma}$ & $5_{\gamma}$ \\
$37_{\gamma}$ & $4_{\gamma}$ \\
$38_{\gamma}$ & $6_{\gamma}$ \\
39 & 13 \\
40 & 14 \\
41 & $16_{+1}$ \\
42 & $16_{-1}$ \\
\hline
\end{tabular}

\begin{tabular}{|l|l|}
\hline$D=2$ & $D \geq 3$ \\
\hline 43 & $15_{+1}$ \\
44 & $15_{-1}$ \\
45 & 17 \\
46 & $18_{+1}$ \\
47 & $18_{-1}$ \\
48 & $19_{-1}$ \\
49 & $19_{+1}$ \\
$50_{\chi}$ & $12_{2 / \chi}$ \\
\hline
\end{tabular}

Table 10. Correspondence between $D=2$ and $D \geq 3$ Lie pairs.

classification, classify the one-dimensional subspaces up to the action of automorphisms. In other words, we must determine the orbits of the action of the automorphism group of $\mathfrak{k}$ on the projective space $\mathbb{P k}$ of one-dimensional subspaces of $\mathfrak{k}$. We will now do this for every Bianchi type in turn. We will choose a basis for $\mathfrak{k}$ where $\mathfrak{h}$ is spanned by $B$ and will choose $P, H$, if possible, so that their span is $\mathfrak{h}$-invariant. The resulting Lie pairs are summarised in table 12 below. Those Lie pairs below the horizontal line correspond to Lie algebras unique to $D=1$; although the Lie pair might actually be the $1+1$ case of a Lie pair which exists for all $D$.

One notational remark about the automorphism groups. Since we have chosen a basis $\left(\boldsymbol{e}_{1}, \boldsymbol{e}_{2}, \boldsymbol{e}_{3}\right)$ for $\mathfrak{k}$, we can identify the automorphism group $A=\operatorname{Aut}(\mathfrak{k})$ with a subgroup of $\mathrm{GL}(3, \mathbb{R})$ and therefore we will be describing it as a set of matrices. 


\begin{tabular}{|c|c|c|c|c|c|}
\hline Bianchi & $\cong$ & \multicolumn{3}{|c|}{ Nonzero brackets } & Comments \\
\hline I & $\mathfrak{s}$ & & & & \\
\hline II & $\mathfrak{g} \cong \mathfrak{c}$ & & & {$\left[e_{2}, e_{3}\right]=e_{1}$} & \\
\hline IV & & & {$\left[e_{1}, e_{3}\right]=e_{1}$} & {$\left[e_{2}, e_{3}\right]=e_{1}+e_{2}$} & \\
\hline V & & & {$\left[e_{1}, e_{3}\right]=e_{1}$} & {$\left[e_{2}, e_{3}\right]=e_{2}$} & \\
\hline $\mathrm{VI}_{0}$ & $\mathfrak{n}_{-} \cong \mathfrak{p}$ & & {$\left[\boldsymbol{e}_{1}, \boldsymbol{e}_{3}\right]=-\boldsymbol{e}_{1}$} & {$\left[\boldsymbol{e}_{2}, \boldsymbol{e}_{3}\right]=\boldsymbol{e}_{2}$} & \\
\hline $\mathrm{VI}_{\chi}$ & & & {$\left[\boldsymbol{e}_{1}, \boldsymbol{e}_{3}\right]=(\chi-1) \boldsymbol{e}_{1}$} & {$\left[\boldsymbol{e}_{2}, \boldsymbol{e}_{3}\right]=(\chi+1) \boldsymbol{e}_{2}$} & $\chi>0$ \\
\hline $\mathrm{VII}_{0}$ & $\mathfrak{n}_{+} \cong \mathfrak{e}$ & & {$\left[e_{1}, e_{3}\right]=-e_{2}$} & {$\left[\boldsymbol{e}_{2}, \boldsymbol{e}_{3}\right]=\boldsymbol{e}_{1}$} & \\
\hline $\mathrm{VII}_{\chi}$ & & & {$\left[\boldsymbol{e}_{1}, \boldsymbol{e}_{3}\right]=\chi \boldsymbol{e}_{1}-\boldsymbol{e}_{2}$} & {$\left[\boldsymbol{e}_{2}, \boldsymbol{e}_{3}\right]=\boldsymbol{e}_{1}+\chi \boldsymbol{e}_{2}$} & $\chi>0$ \\
\hline VIII & $\mathfrak{s o}(1,2)$ & {$\left[e_{1}, e_{2}\right]=-e_{3}$} & {$\left[\boldsymbol{e}_{1}, \boldsymbol{e}_{3}\right]=-\boldsymbol{e}_{2}$} & {$\left[\boldsymbol{e}_{2}, \boldsymbol{e}_{3}\right]=\boldsymbol{e}_{1}$} & \\
\hline IX & $\mathfrak{s o}(3)$ & {$\left[e_{1}, e_{2}\right]=e_{3}$} & {$\left[\boldsymbol{e}_{1}, \boldsymbol{e}_{3}\right]=-\boldsymbol{e}_{2}$} & {$\left[\boldsymbol{e}_{2}, \boldsymbol{e}_{3}\right]=\boldsymbol{e}_{1}$} & \\
\hline
\end{tabular}

Table 11. Kinematical Lie algebras for $D=1$.

\subsubsection{Bianchi I}

Since $\mathfrak{k}$ is abelian, every invertible linear map is an automorphism. The general linear group acts transitively on the projective space, so we can take $\mathfrak{h}$ to be spanned by $B=\boldsymbol{e}_{1}$, say.

\subsubsection{Bianchi II}

Here $\mathfrak{k}$ is the Heisenberg algebra, whose automorphisms can be determined to be

$$
A=\left\{\left(\begin{array}{ccc}
a d-b c & \alpha & \beta \\
0 & a & b \\
0 & c & d
\end{array}\right) \quad a, b, c, d, \alpha, \beta \in \mathbb{R}, a d \neq b c\right\} .
$$

A nonzero vector $x^{1} \boldsymbol{e}_{1}+x^{2} \boldsymbol{e}_{2}+x^{3} \boldsymbol{e}_{3}$ determines a one-dimensional subspace and hence an admissible subalgebra. Under a typical automorphism, this vector transforms as

$$
\left(\begin{array}{l}
x^{1} \\
x^{2} \\
x^{3}
\end{array}\right) \mapsto\left(\begin{array}{ccc}
a d-b c & \alpha & \beta \\
0 & a & b \\
0 & c & d
\end{array}\right)\left(\begin{array}{l}
x^{1} \\
x^{2} \\
x^{3}
\end{array}\right)=\left(\begin{array}{c}
(a d-b c) x^{1}+\alpha x^{2}+\beta x^{3} \\
a x^{2}+b x^{3} \\
c x^{2}+d x^{3}
\end{array}\right)
$$

The line spanned by $\boldsymbol{e}_{1}$ is sent to itself, whereas any other line can be transformed to the line spanned by $\boldsymbol{e}_{2}$, say.

In the first case we let $B=\boldsymbol{e}_{1}, P=\boldsymbol{e}_{2}$ and $H=\boldsymbol{e}_{3}$, arriving at the Lie algebra with nonzero brackets

$$
[P, H]=B .
$$

In the second case we let $B=\boldsymbol{e}_{2}, P=\boldsymbol{e}_{1}$ and $H=\boldsymbol{e}_{3}$, arriving at

$$
[B, H]=P .
$$

\subsubsection{Bianchi IV}

The automorphism group is now

$$
A=\left\{\left(\begin{array}{lll}
a & b & \alpha \\
0 & a & \beta \\
0 & 0 & 1
\end{array}\right) \mid a, b, \alpha, \beta \in \mathbb{R}, a \neq 0\right\} .
$$

There are three $A$-orbits in $\mathbb{P k}$, labelled by the vectors $(1,0,0),(0,1,0)$ and $(0,0,1)$. 
In the first case, $B=\boldsymbol{e}_{1}, P=\boldsymbol{e}_{2}$ and $H=\boldsymbol{e}_{3}$, with nonzero brackets

$$
[B, H]=B \quad \text { and } \quad[P, H]=B+P .
$$

In the second case, $B=\boldsymbol{e}_{2}, P=\boldsymbol{e}_{1}$ and $H=\boldsymbol{e}_{3}$, with nonzero brackets

$$
[B, H]=P \quad \text { and } \quad[P, H]=2 P-B .
$$

In the final case, $B=\boldsymbol{e}_{3}, P=\boldsymbol{e}_{1}$ and $H=\boldsymbol{e}_{2}$, with nonzero brackets

$$
[B, P]=-P \quad \text { and } \quad[B, H]=-P-H .
$$

\subsubsection{Bianchi V}

The automorphism group in this case is

$$
A=\left\{\left(\begin{array}{lll}
a & b & \alpha \\
c & d & \beta \\
0 & 0 & 1
\end{array}\right) \mid a, b, c, d, \alpha, \beta \in \mathbb{R}, a d \neq b c\right\} .
$$

There are two $A$-orbits in $\mathbb{P k}$ labelled by the vectors $(1,0,0)$ and $(0,0,1)$.

In the first case, $B=e_{1}, P=e_{2}$ and $H=e_{3}$, with nonzero brackets

$$
[B, H]=B \quad \text { and } \quad[P, H]=P .
$$

In the other case, $B=e_{3}, P=e_{1}$ and $H=e_{2}$, with nonzero brackets

$$
[B, P]=-P \quad \text { and } \quad[B, H]=-H .
$$

\subsubsection{Bianchi $\mathrm{VI}_{0}$}

The automorphism group is this case is

$$
\begin{aligned}
A=\left\{\left(\begin{array}{lll}
a & 0 & \alpha \\
0 & d & \beta \\
0 & 0 & 1
\end{array}\right) \mid a, d, \alpha, \beta \in \mathbb{R}, a d \neq 0\right\} & \\
& \bigcup\left\{\left(\begin{array}{ccc}
0 & b & \alpha \\
c & 0 & \beta \\
0 & 0 & -1
\end{array}\right) \mid b, c, \alpha, \beta \in \mathbb{R}, b c \neq 0\right\} .
\end{aligned}
$$

There are three $A$-orbits in $\mathbb{P k}$, labelled by $(0,0,1),(1,0,0)$ and $(1,1,0)$.

In the first case, $B=e_{3}, P=e_{1}$ and $H=e_{2}$, with nonzero brackets

$$
[B, H]=-H \quad \text { and } \quad[B, P]=P .
$$

In the second case, $B=e_{1}, P=e_{2}$ and $H=e_{3}$, with nonzero brackets

$$
[B, H]=-B \quad \text { and } \quad[P, H]=P .
$$

This spacetime is not reductive.

In the final case, $B=\boldsymbol{e}_{1}+\boldsymbol{e}_{2}, P=-\boldsymbol{e}_{1}+\boldsymbol{e}_{2}$ and $H=\boldsymbol{e}_{3}$, with nonzero brackets

$$
[B, H]=P \quad \text { and } \quad[P, H]=B .
$$




\subsubsection{Bianchi $\mathrm{VI}_{\chi>0}$}

The automorphism group in this case is the identity component of the automorphism group of Bianchi $\mathrm{VI}_{0}$ :

$$
A=\left\{\left(\begin{array}{lll}
a & 0 & \alpha \\
0 & d & \beta \\
0 & 0 & 1
\end{array}\right) \quad a, d, \alpha, \beta \in \mathbb{R}, a d \neq 0\right\} .
$$

There are four $A$-orbits in $\mathbb{P k}^{\mathfrak{k}}$ labelled by the vectors $(0,0,1),(1,0,0),(0,1,0)$ and $(1,1,0)$.

In the first case, $B=e_{3}, P=e_{1}$ and $H=e_{2}$, with nonzero brackets

$$
[B, H]=-(1+\chi) H \quad \text { and } \quad[B, P]=(1-\chi) P .
$$

In the second case, $B=e_{1}, P=e_{2}$ and $H=e_{3}$, with nonzero brackets

$$
[B, H]=(\chi-1) B \quad \text { and } \quad[P, H]=(\chi+1) P .
$$

In the third case, $B=\boldsymbol{e}_{2}, P=\boldsymbol{e}_{1}$ and $H=\boldsymbol{e}_{3}$, with nonzero brackets

$$
[B, H]=(\chi+1) B \quad \text { and } \quad[P, H]=(\chi-1) P .
$$

In the final case, $B=e_{1}+e_{2}, P=e_{2}-e_{1}$ and $H=e_{3}$, with nonzero brackets

$$
[B, H]=\chi B+P \quad \text { and } \quad[P, H]=\chi P+B .
$$

\subsubsection{Bianchi $\mathrm{VII}_{0}$}

The automorphism group has two connected components:

$$
\begin{aligned}
A=\left\{\left(\begin{array}{ccc}
a & b & \alpha \\
-b & a & \beta \\
0 & 0 & 1
\end{array}\right)\right. & \left.\mid a, b, \alpha, \beta \in \mathbb{R}, a^{2}+b^{2} \neq 0\right\} \\
& \bigcup\left\{\left(\begin{array}{ccc}
a & b & \alpha \\
b & -a & \beta \\
0 & 0 & -1
\end{array}\right) \mid a, b, \alpha, \beta \in \mathbb{R}, a^{2}+b^{2} \neq 0\right\} .
\end{aligned}
$$

Using only the identity component, we can bring any line in $\mathbb{P k}$ to of of two lines: the one spanned by $(0,0,1)$ and the one spanned by $(1,0,0)$.

In the first case, $B=e_{3}, P=e_{1}$ and $H=e_{2}$, with nonzero brackets

$$
[B, H]=-P \quad \text { and } \quad[B, P]=H .
$$

In the other case, $B=\boldsymbol{e}_{1}, P=\boldsymbol{e}_{2}$ and $H=\boldsymbol{e}_{3}$, with nonzero brackets

$$
[B, H]=-P \quad \text { and } \quad[P, H]=B .
$$




\subsubsection{Bianchi $\mathrm{VII}_{\chi>0}$}

The automorphism group in this case is the identity component of the automorphism group for Bianchi VII :

$$
A=\left\{\left(\begin{array}{ccc}
a & b & \alpha \\
-b & a & \beta \\
0 & 0 & 1
\end{array}\right) \quad a, b, \alpha, \beta \in \mathbb{R}, a^{2}+b^{2} \neq 0\right\} .
$$

This was all that was needed to bring every line to one of two lines, spanned by either $(0,0,1)$ and $(1,0,0)$.

In the first case, $B=e_{3}, P=e_{1}$ and $H=e_{2}$, with nonzero brackets

$$
[B, H]=-P-\chi H \quad \text { and } \quad[B, P]=H-\chi P .
$$

In the other case, $B=e_{1}, P=e_{2}$ and $H=e_{3}$, with nonzero brackets

$$
[B, H]=\chi B-P \quad \text { and } \quad[P, H]=B+\chi P .
$$

\subsubsection{Bianchi VIII}

This Lie algebra is isomorphic to $\mathfrak{s o}(2,1)$ and the automorphism group is the adjoint group, so isomorphic to $\mathrm{SO}(2,1)$. It acts on $\mathfrak{k}$ via proper Lorentz transformations and hence has three orbits in the space of lines, corresponding to timelike, spacelike and null lines. The inner product on $\mathfrak{k}$ is the Killing form $\kappa$, which in the chosen basis is diagonal with components $\kappa\left(\boldsymbol{e}_{1}, \boldsymbol{e}_{1}\right)=\kappa\left(\boldsymbol{e}_{2}, \boldsymbol{e}_{2}\right)=2$ and $\kappa\left(\boldsymbol{e}_{3}, \boldsymbol{e}_{3}\right)=-2$.

We can take for our spacelike line, the one spanned by $(1,0,0)$. Here $B=\boldsymbol{e}_{1}, P=\boldsymbol{e}_{2}$ and $H=e_{3}$, with brackets

$$
[B, H]=-P, \quad[B, P]=-H \quad \text { and } \quad[P, H]=B .
$$

For the timelike line, we take the one spanned by $(0,0,1)$. Here $B=\boldsymbol{e}_{3}, P=\boldsymbol{e}_{1}$ and $H=e_{2}$, with brackets

$$
[B, H]=-P, \quad[B, P]=H \quad \text { and } \quad[P, H]=-B .
$$

Finally, we take the span of $(1,0,1)$ for the null line. Here $B=\boldsymbol{e}_{1}+\boldsymbol{e}_{3}, P=\boldsymbol{e}_{2}$ and $H=e_{1}-e_{3}$, with brackets

$$
[B, H]=2 P, \quad[B, P]=-B \quad \text { and } \quad[P, H]=-H .
$$

\subsubsection{Bianchi IX}

Bianchi IX is isomorphic to $\mathfrak{s u}(2)$, whose automorphism group is the adjoint group $\mathrm{SO}(3)$, which acts transitively on the space of lines. We can take $B=\boldsymbol{e}_{1}, P=\boldsymbol{e}_{2}$ and $H=\boldsymbol{e}_{3}$ with brackets

$$
[B, H]=-P, \quad[B, P]=H \quad \text { and } \quad[P, H]=B .
$$




\begin{tabular}{|c|c|c|c|c|c|}
\hline LP\# & Bianchi & & Nonzero Lie brac & zets & Comments \\
\hline 61 & I & & & & static \\
\hline 62 & II & & & {$[H, P]=B$} & \\
\hline 63 & II & {$[H, B]=-P$} & & & galilean/carrollian \\
\hline 64 & IV & {$[H, B]=B$} & & {$[H, P]=B+P$} & \\
\hline 65 & IV & {$[H, B]=-P$} & & {$[H, P]=B+2 P$} & \\
\hline 66 & $\mathrm{~V}$ & {$[H, B]=B$} & & {$[H, P]=P$} & \\
\hline 67 & $\mathrm{VI}_{0}$ & {$[H, B]=-B$} & & {$[H, P]=P$} & \\
\hline 68 & $\mathrm{VI}_{0}$ & {$[H, B]=-P$} & & {$[H, P]=-B$} & galilean dS/carrollian AdS \\
\hline $69 x$ & $\mathrm{VI}_{\chi}$ & {$[H, B]=(\chi-1) B$} & & {$[H, P]=(1+\chi) P$} & $\chi>0$ \\
\hline $70_{\chi}$ & $\mathrm{VI}_{\chi}$ & {$[H, B]=(\chi+1) B$} & & {$[H, P]=(\chi-1) P$} & $\chi>0$ \\
\hline $71_{\chi}$ & $\mathrm{VI}_{\chi}$ & {$[H, B]=-\chi B+P$} & & {$[H, P]=B-\chi P$} & $\chi>0$ \\
\hline 72 & $\mathrm{VII}_{0}$ & {$[H, B]=P$} & {$[B, P]=H$} & & euclidean \\
\hline 73 & $\mathrm{VII}_{0}$ & {$[H, B]=-P$} & & {$[H, P]=B$} & galilean AdS/carrollian dS \\
\hline $74_{\chi}$ & $\mathrm{VI}_{\chi}$ & {$[H, B]=-P$} & & {$[H, P]=\left(1+\chi^{2}\right) B+2 \chi P$} & $\chi>0$ \\
\hline 75 & VIII & {$[H, B]=-P$} & {$[B, P]=H$} & {$[H, P]=B$} & (anti) de Sitter \\
\hline 76 & VIII & {$[H, B]=P$} & {$[B, P]=H$} & {$[H, P]=B$} & hyperbolic \\
\hline 77 & IX & {$[H, B]=P$} & {$[B, P]=H$} & {$[H, P]=-B$} & sphere \\
\hline 78 & $\mathrm{VI}_{0}$ & {$[H, B]=H$} & {$[B, P]=P$} & & Minkowski \\
\hline 79 & VIII & {$[H, B]=-P$} & {$[B, P]=B$} & {$[H, P]=-H$} & \\
\hline 80 & IV & {$[H, B]=P+H$} & {$[B, P]=-P$} & & \\
\hline 81 & $\mathrm{~V}$ & {$[H, B]=H$} & {$[B, P]=-P$} & & \\
\hline $82 \chi$ & $\mathrm{VI}_{\chi}$ & {$[H, B]=(1+\chi) H$} & {$[B, P]=(1-\chi) P$} & & $\chi>0$ \\
\hline $83_{\chi}$ & $\mathrm{VII}_{\chi}$ & {$[H, B]=P+\chi H$} & {$[B, P]=H-\chi P$} & & $\chi>0$ \\
\hline
\end{tabular}

Table 12. Lie pairs for kinematical Lie algebras $(D=1)$.

\subsubsection{Summary}

In table 12 we list the Lie pairs associated to the Bianchi Lie algebras. In that table we have often redefined $H$ and $P$ linearly in order to ease the comparison with the Lie pairs in $D \geq 3$. This comparison leads to a division of the table into two: the ones above the horizontal line are $D=1$ avatars of Lie pairs which exist also for $D>1$. Table 13 shows the correspondence between the $D=1$ Lie pairs and the $D \geq 3$ Lie pairs in those cases where there is one. Notice that in $D=1$ there are exceptional isomorphisms between Lie pairs which might differ in $D \geq 3$. For example, exchanging $P$ and $H$ (i.e., re-interpreting what is time and space), we see that the following pairs of spacetimes are isomorphic: de Sitter/anti de Sitter, galilean/carrollian, galilean dS/carrollian AdS and galilean AdS/carrollian dS.

As in previous tables (tables 5, 7 and 9) each row in table 12 is an isomorphism class of kinematical Lie pair with sequential label "LP\#". Similarly, the label "Bianchi" in table 12 identifies the Bianchi type of the Lie algebra in table 11. It bears reminding that the Lie brackets in the tables below are expressed in a basis where the admissible subalgebra $\mathfrak{h}$ is spanned by $B$.

\section{Classification of simply-connected homogeneous spacetimes}

In section 3 we have classified the equivalence classes of Lie pairs $(\mathfrak{k}, \mathfrak{h})$ where $\mathfrak{k}$ is a kinematical Lie algebra and $\mathfrak{h}$ is an admissible subalgebra. Our aim is to classify simply-connected homogeneous spacetimes and, as explained in appendix B, this requires selecting those Lie pairs which are effective (so that $\mathfrak{h}$ contains no nonzero ideal of $\mathfrak{k}$ ) and also geometrically 


\begin{tabular}{|l|l|}
\hline$D=1$ & $D \geq 3$ \\
\hline 61 & 1 \\
62 & 3 \\
63 & 2 and 14 \\
64 & 11 \\
65 & 10 \\
66 & 7 \\
\hline
\end{tabular}

\begin{tabular}{|l|l|}
\hline$D=1$ & \multicolumn{1}{|c|}{$D \geq 3$} \\
\hline 67 & 8 \\
68 & 9 and $16_{+1}$ \\
$69_{\chi}$ & $4_{\frac{\chi-1}{\chi+1}}$ \\
$70_{\chi}$ & $5_{\frac{\chi-1}{\chi+1}}$ \\
$71_{\chi}$ & $6_{\frac{\chi-1}{\chi+1}}$ \\
72 & $15_{-1}$ \\
\hline
\end{tabular}

\begin{tabular}{|l|l|}
\hline$D=1$ & \multicolumn{1}{|c|}{$D \geq 3$} \\
\hline 73 & 13 and $16_{-1}$ \\
$74_{\chi}$ & $12_{\chi}$ \\
75 & $18_{-1}$ and $19_{+1}$ \\
76 & $18_{+1}$ \\
77 & $19_{-1}$ \\
78 & $15_{+1}$ \\
79 & 17 \\
\hline
\end{tabular}

Table 13. Correspondence between $D=1$ and $D \geq 3$ Lie pairs.

realisable, so that there exists a kinematical Lie group $\mathcal{K}^{\prime}$ and an admissible subgroup $\mathcal{H}^{\prime}$ whose Lie pair $\left(\mathfrak{k}^{\prime}, \mathfrak{h}^{\prime}\right)$ is isomorphic to $(\mathfrak{k}, \mathfrak{h})$. Let us discuss both of these selection criteria in turn.

\subsection{Effective Lie pairs}

Recall that we have chosen a basis for the kinematical Lie algebra $\mathfrak{k}$ such that the subalgebra $\mathfrak{h}$ is spanned by $J_{a b}$ and $B_{a}$. This means that the only possible nonzero ideal of $\mathfrak{k}$ contained in $\mathfrak{h}$ is the span of the boosts $B_{a}$. Therefore to check if a Lie pair $(\mathfrak{k}, \mathfrak{h})$ is effective, all we need to do is inspect the Lie brackets $\left[B_{a}, X\right]$ for $X$ in the span of $\left(B_{a}, P_{a}, H\right)$ and see whether they all lie in the span of the $B_{a}$ (in which case the Lie pair is not effective) or not (in which case it is). Reducing a non-effective Lie pair by the ideal $\mathfrak{b}$ spanned by the boosts, we arrive at an effective aristotelian Lie pair. Since it turns out that not all aristotelian Lie pairs arise in this way, we treat their classification separately in appendix A.

\subsubsection{Effective Lie pairs for all $D \geq 3$}

Inspecting table 5 we see that Lie pairs $1,3,4_{\gamma}, 5_{\gamma}, 7,8$ and 11 are not effective, since the span of the $B_{a}$ define an ideal of $\mathfrak{k}$. Reducing by that ideal we obtain an aristotelian Lie pair of the ones in table 2, which are classified in appendix A:

- Lie pairs 1 and 3 reduce to the static aristotelian Lie pair A21; whereas

- Lie pairs $4_{\gamma}, 5_{\gamma}, 7,8$ and 11 reduce to the torsional static aristotelian Lie pair A22.

\subsubsection{No effective Lie pairs unique to $D=3$}

Inspecting table 7 we see that no Lie pairs for kinematical Lie algebras unique to $D=3$ are effective. They all reduce to aristotelian Lie pairs:

- Lie pairs 21, 23 and 26 reduce to the static aristotelian Lie pair A21;

- Lie pairs 24 and 27 reduce to the torsional static aristotelian Lie pair A22;

- Lie pair $20_{\varepsilon}$ reduces to the aristotelian Lie pair A23 $\varepsilon_{\varepsilon}$ with bracket $[\boldsymbol{P}, \boldsymbol{P}]=-\varepsilon \boldsymbol{J}$; and

- Lie pairs 22 and 25 reduce to $[\boldsymbol{P}, \boldsymbol{P}]=\boldsymbol{P}$, which is isomorphic to A23 $3_{+1}$ after changing basis $P_{a} \mapsto \frac{1}{2}\left(P_{a}+\frac{1}{4} \epsilon_{a b c} J_{b c}\right)$. 


\subsubsection{Effective Lie pairs for $D=2$}

We inspect table 9 and concentrate on the Lie pairs which are unique to $D=2$, since for those which exist for $D>2$ the calculations for $D>2$ are valid also for $D=2$. We see that only the Lie pair $55_{\gamma, \chi}$ is effective. The others reduce to aristotelian Lie pairs:

- 56 to A21;

- $51_{\chi}, 52_{\chi}, 53_{\gamma, \chi}$ and $54_{\gamma, \chi}$ to A22;

- 57 and 58 to A24; and

- 59 and 60 , respectively, to the aristotelian Lie pairs A23-1 and A23+1, after redefining $H$ and $J$.

\subsubsection{Effective Lie pairs for $D=1$}

Inspecting table 12 , but concentrating only on the Lie pairs unique to $D=1$ (those below the horizontal line), we see that they are all effective.

\subsubsection{Summary}

Table 14 summarises the effective Lie pairs. Some classes of Lie pairs exist for all $D \geq 1$ and we collect them in the same row. This is possibly the most navigationally useful table in the paper, in that it shows the correspondence between the spacetimes and their Lie pairs. The table is hyperlinked for ease of navigation.

Table 15 is included for convenience. We have found this list useful at times and thus we think it might be useful to other readers. Table 15 lists which simply-connected homogeneous kinematical or aristotelian spacetimes are associated to which kinematical Lie algebras. We do not list aristotelian Lie algebras since there is a one-to-one correspondence between the Lie algebras and the simply-connected homogenous aristotelian spacetimes in that case. The table is separated into three: corresponding to the Lie algebras for $D \geq 3$, $D=2$ and $D=1$. The Lie algebras below the horizontal line in the first part of the table exist only for $D=3$. Lie algebras 29 and 30, which exist only in $D=2$, have no admissible Lie pairs at all.

\subsection{Geometric realisability}

Having selected the effective Lie pairs, we must then select those which are geometrically realisable, so that they correspond to the Lie pair of a homogeneous spacetime. It will turn out that all effective Lie pairs are geometrically realisable. Recall that $(\mathfrak{k}, \mathfrak{h})$ is geometrically realisable if there exists a connected Lie group $\mathcal{K}$, a Lie algebra isomorphism $\varphi: \operatorname{Lie}(\mathcal{K}) \rightarrow \mathfrak{k}$ and a closed Lie subgroup $\mathcal{H} \subset \mathcal{K}$ whose Lie algebra is isomorphic to $\mathfrak{h}$ under $\varphi$. There are a number of criteria which can be brought to bear in order to help decide whether a Lie pair admits a geometric realisation. 


\begin{tabular}{|c|c|c|c|c|}
\hline Label & $D \geq 3$ & $D=2$ & $D=1$ & Comments \\
\hline S1 & $15_{+1}$ & 43 & 78 & Minkowski (M) \\
\hline S2 & $18_{-1}$ & 47 & 75 & de Sitter (dS) \\
\hline S3 & $19+1$ & 49 & 75 & anti de Sitter (AdS) \\
\hline $\mathrm{S} 4$ & $15_{-1}$ & 44 & 72 & euclidean $(\mathbb{E})$ \\
\hline S5 & $19_{-1}$ & 48 & 77 & sphere $(\mathbb{S})$ \\
\hline $\mathrm{S} 6$ & $18_{+1}$ & 46 & 76 & hyperbolic $(\mathbb{H})$ \\
\hline S7 & 2 & 29 & 63 & galilean $(\mathrm{G})$ \\
\hline S8 & 9 & 35 & 68 & galilean $\mathrm{dS}\left(\mathrm{dSG}=\mathrm{dSG}_{-1}\right)$ \\
\hline $\mathrm{S} 9_{\gamma}$ & $6_{\gamma}$ & $38_{\gamma}$ & $71_{\frac{1+\gamma}{1-\gamma}}$ & $\mathrm{dSG}_{\gamma \in(-1,1)}$ \\
\hline $\mathrm{S} 9_{1}$ & 10 & 31 & 65 & $\mathrm{dSG}_{1}=\mathrm{AdSG}_{\infty}$ \\
\hline S10 & 13 & 39 & 73 & galilean $\mathrm{AdS}\left(\mathrm{AdSG}=\mathrm{AdSG}_{0}\right)$ \\
\hline $\mathrm{S} 11_{\chi}$ & $12_{\chi}$ & $50_{\frac{2}{x}}$ & $74 \chi$ & $\operatorname{AdSG}_{\chi>0}$ \\
\hline $\mathrm{S} 12_{\gamma, \chi}$ & - & $55_{\gamma, \chi}$ & - & $\gamma \in[-1,1), \chi>0$ \\
\hline S13 & 14 & 40 & 63 & carrollian (C) \\
\hline S14 & $16_{-1}$ & 42 & 73 & carrollian dS (dSC) \\
\hline S15 & $16_{+1}$ & 41 & 68 & carrollian AdS (AdSC) \\
\hline S16 & 17 & 45 & 79 & carrollian light cone (LC) \\
\hline S17 & - & - & 80 & \\
\hline S18 & - & - & 81 & \\
\hline $\mathrm{S} 19_{\chi}$ & - & - & $82 \chi$ & $\chi>0$ \\
\hline $\mathrm{S} 20_{\chi}$ & - & - & $83_{\chi}$ & $\chi>0$ \\
\hline
\end{tabular}

Table 14. Effective kinematical Lie pairs.

\begin{tabular}{|c|c|c|c|}
\hline Lie algebra & \multicolumn{3}{|c|}{ Spacetimes } \\
\hline 1 & A21 & & \\
\hline 2 & S7 & A21 & \\
\hline $3_{\gamma}$ & $\mathrm{S} 9_{\gamma}$ & A22 & \\
\hline 4 & A22 & & \\
\hline 5 & S8 & A22 & \\
\hline 6 & $\mathrm{~S} 9_{1}$ & A22 & \\
\hline $7_{\chi}$ & $\mathrm{S} 11_{\chi}$ & & \\
\hline 8 & $\mathrm{~S} 10$ & & \\
\hline 9 & S13 & & \\
\hline $10_{+1}$ & S1 & $\mathrm{S} 15$ & \\
\hline $10_{-1}$ & $\mathrm{~S} 4$ & S14 & \\
\hline 11 & $\mathrm{~S} 2$ & S6 & S16 \\
\hline $12_{+1}$ & S3 & & \\
\hline $12_{-1}$ & S5 & & \\
\hline $13_{\varepsilon}$ & $\mathrm{A} 23_{\varepsilon}$ & & \\
\hline 14 & A21 & $\mathrm{A} 23_{+1}$ & \\
\hline 15 & A21 & & \\
\hline 16 & A22 & $\mathrm{A} 23_{+1}$ & \\
\hline 17 & A21 & & \\
\hline 18 & $\mathrm{~A} 22$ & & \\
\hline
\end{tabular}

\begin{tabular}{|l|ll|}
\hline Lie algebra & \multicolumn{2}{|c|}{ Spacetimes } \\
\hline 19 & A21 & \\
20 & S7 & A21 \\
$22_{1+i 0}$ & S ${ }_{1}$ & A22 \\
$22_{\gamma+i 0}$ & A22 & \\
$22_{1+i \chi}$ & S $9_{\gamma}$ & A22 \\
$22_{\gamma+i \chi}$ & S11 & A22 \\
23 & S12 & A22 \\
24 & S 8 & A22 \\
25 & S10 & A21 \\
$26_{+1}$ & S13 & \\
$26_{-1}$ & S1 & S15 \\
27 & S4 & S14 \\
$28_{+1}$ & S2 & S6 \\
$28_{-1}$ & S 5 & \\
31 & S3 & \\
32 & A24 & \\
33 & A23 & \\
\hline
\end{tabular}

\begin{tabular}{|c|llll|}
\hline Lie algebra & \multicolumn{5}{|c|}{ Spacetimes } \\
\hline I & $\mathrm{A} 21$ & & & \\
II & $\mathrm{S} 7$ & $\mathrm{~S} 13$ & $\mathrm{~A} 21$ & \\
IV & $\mathrm{S} 9_{1}$ & $\mathrm{~S} 17$ & $\mathrm{~A} 22$ & \\
V & $\mathrm{S} 18$ & & & \\
$\mathrm{VI}_{0}$ & $\mathrm{~S} 1$ & $\mathrm{~S} 8$ & $\mathrm{~S} 15$ & $\mathrm{~A} 22$ \\
$\mathrm{VI}_{\chi}$ & $\mathrm{S} 9_{\frac{\chi-1}{\chi+1}}$ & $\mathrm{~S} 11_{\chi}$ & $\mathrm{S} 19_{\chi}$ & $\mathrm{A} 22$ \\
$\mathrm{VII}_{0}$ & $\mathrm{~S} 4$ & $\mathrm{~S} 10$ & $\mathrm{~S} 14$ & \\
$\mathrm{VII}_{\chi}$ & $\mathrm{S} 20_{\chi}$ & & & \\
$\mathrm{VIII}$ & $\mathrm{S} 2$ & $\mathrm{~S} 3$ & $\mathrm{~S} 6$ & $\mathrm{~S} 16$ \\
$\mathrm{IX}$ & $\mathrm{S} 5$ & & & \\
\hline
\end{tabular}

Table 15. Kinematical Lie algebras and their homogeneous spacetimes. 


\subsubsection{Riemannian maximally symmetric spaces}

We start by showing that the Lie pairs corresponding to the riemannian symmetric spaces are geometrically realisable; although this is of course well-known.

Criterion 1. Compact subgroups are closed, so if if $\mathfrak{h}$ generates a compact subgroup, then $(\mathfrak{k}, \mathfrak{h})$ is geometrically realisable.

From this criterion we see that the following Lie pairs are geometrically realisable:

- Lie pairs $15_{-1}, 44$ and 72 , which we can identify with euclidean spaces;

- Lie pairs $18_{+1}, 46,76$, which we can identify with hyperbolic spaces; and

- Lie pairs $19_{-1}, 48,77$, which we can identify with the round spheres.

\subsubsection{A sufficient criterion}

Another useful criterion (sufficient, but by no means necessary) applies to linear Lie algebras; that is, Lie algebras isomorphic to Lie algebras of matrices. By Ado's theorem (see, e.g., [51, chapter VI]) every kinematical Lie algebra $\mathfrak{k}$, being finite-dimensional, has a faithful linear representation and hence is a linear Lie algebra. Exponentiating inside the matrix algebra, we obtain a connected Lie group $\mathcal{K}$ with $\mathfrak{k}$ (or, more precisely, its isomorphic image in the matrix algebra) as its Lie algebra.

Criterion 2. If the subalgebra $\mathfrak{h}$ of a linear Lie algebra $\mathfrak{k}$ is its own normaliser, so that the only elements $X \in \mathfrak{k}$ with $[X, \mathfrak{h}] \subset \mathfrak{h}$ are the elements of $\mathfrak{h}$, then the unique connected subgroup $\mathcal{H} \subset \mathcal{K}$ to which it exponentiates is closed (see, e.g., [52, Pr. 2.7.4]) and $\mathcal{K} / \mathcal{H}$ is geometric realisation of $(\mathfrak{k}, \mathfrak{h})$.

In particular, if the Lie pair is reductive, so that $\mathfrak{k}=\mathfrak{h} \oplus \mathfrak{m}$ as adjoint $\mathfrak{h}$-modules, then we may decompose $X \in \mathfrak{k}$ uniquely as $X=X_{\mathfrak{h}}+X_{\mathfrak{m}}$, with $X_{\mathfrak{h}} \in \mathfrak{h}$ and $X_{\mathfrak{m}} \in \mathfrak{m}$. Now, rotations (when present) act reducibly, so $\left[J_{a b}, X\right] \in \mathfrak{h}$ means that $X_{\mathfrak{m}}=c H$ for some $c$. So we then need to inspect whether $\left[B_{a}, H\right] \in \mathfrak{h}$.

As we can see by inspection, this second criterion allows us to conclude that all Lie pairs are geometrically realisable with the following possible exceptions requiring a closer look: Lie pairs $14,16_{\varepsilon}$ and 17 in $D \geq 3$, Lie pairs $40,41,42$ and 45 in $D=2$ and Lie pairs $63,65,68,71_{\chi}, 73,74_{\chi}, 79$ and $82_{\chi=1}$ in $D=1$.

\subsubsection{Two-dimensional spacetimes}

To show that $D=1$ Lie pairs $63,65,68,71_{\chi}, 73,74_{\chi}, 79$ and $82_{\chi=1}$ are geometrically realisable, we may use yet a third criterion for when a one-dimensional subgroup of a matrix group is (not) closed.

Criterion 3. A one-parameter subgroup of a matrix group is not closed if and only if the generating matrix in the Lie algebra is similar to a diagonal matrix with imaginary entries, at least two of which have an irrational ratio (see, e.g., [52, Pr. 2.7.5]). 
All the Bianchi Lie algebras have faithful representations of dimension 2 or 3 . So it is simply a matter of calculating the eigenvalues of $B$ in each of these representations to deduce that all these Lie pairs are geometrically realisable. Indeed, let us go back to the description of the Bianchi Lie algebras in terms of the basis $\left(\boldsymbol{e}_{1}, \boldsymbol{e}_{2}, \boldsymbol{e}_{3}\right)$ as in table 11 and let us write the generic element as $X=x \boldsymbol{e}_{1}+y \boldsymbol{e}_{2}+z \boldsymbol{e}_{3}$. For each of the cases of interest, we will write down the matrix $\rho(X)$ representing $X$ and the matrix $\rho(B)$ representing $B$. We will see that in no case does $\rho(B)$ have imaginary eigenvalues.

(63) This is Bianchi II,

$$
\rho(X)=\left(\begin{array}{ccc}
0 & -z & x \\
0 & 0 & y \\
0 & 0 & 0
\end{array}\right) \quad \text { so that } \quad \rho(B)=\rho\left(\boldsymbol{e}_{2}\right)=\left(\begin{array}{lll}
0 & 0 & 0 \\
0 & 0 & 1 \\
0 & 0 & 0
\end{array}\right) .
$$

This matrix is not diagonalisable.

(65) This is Bianchi IV,

$$
\rho(X)=\left(\begin{array}{ccc}
-z & -z & x \\
0 & -z & y \\
0 & 0 & 0
\end{array}\right) \quad \text { so that } \quad \rho(B)=\rho\left(\boldsymbol{e}_{2}\right)=\left(\begin{array}{lll}
0 & 0 & 0 \\
0 & 0 & 1 \\
0 & 0 & 0
\end{array}\right),
$$

which is not diagonalisable.

(68) This is Bianchi $\mathrm{VI}_{0}$, but let us consider the general Bianchi $\mathrm{VI}_{\chi}$,

$\rho(X)=\left(\begin{array}{ccc}(1-\chi) z & 0 & x \\ 0 & -(1+\chi) z & y \\ 0 & 0 & 0\end{array}\right) \quad$ so that $\quad \rho(B)=\rho\left(\boldsymbol{e}_{1}+\boldsymbol{e}_{2}\right)=\left(\begin{array}{lll}0 & 0 & 1 \\ 0 & 0 & 1 \\ 0 & 0 & 0\end{array}\right)$,

which is not diagonalisable.

$\left(71_{\chi}\right)$ This is again Bianchi $\mathrm{VI}_{\chi}$, so that we can reuse the previous calculation. In this case also

$$
\rho(B)=\rho\left(\boldsymbol{e}_{1}+\boldsymbol{e}_{2}\right)=\left(\begin{array}{lll}
0 & 0 & 1 \\
0 & 0 & 1 \\
0 & 0 & 0
\end{array}\right)
$$

which is not diagonalisable.

$\left(74_{\chi}\right)$ This is again Bianchi $\mathrm{VI}_{\chi}$ and again $B=\boldsymbol{e}_{1}+\boldsymbol{e}_{2}$, with the same matrix nondiagonalisable as above.

(73) This is Bianchi $\mathrm{VII}_{0}$, but we will treat the general Bianchi $\mathrm{VII}_{\chi}$ whose matrix representation is

$$
\rho(X)=\left(\begin{array}{ccc}
-\chi z & -z & x \\
z & -\chi z & y \\
0 & 0 & 0
\end{array}\right) \quad \text { so that } \quad \rho(B)=\rho\left(\boldsymbol{e}_{1}\right)=\left(\begin{array}{lll}
0 & 0 & 1 \\
0 & 0 & 0 \\
0 & 0 & 0
\end{array}\right),
$$

which is not diagonalisable. 
$\left(82_{1}\right)$ This is Bianchi $\mathrm{VII}_{1}$ and $B=\boldsymbol{e}_{3}$, so that the matrix representing it is

$$
\rho(B)=\rho\left(\boldsymbol{e}_{1}\right)=\left(\begin{array}{ccc}
-1 & -1 & 0 \\
1 & -1 & 0 \\
0 & 0 & 0
\end{array}\right) .
$$

This matrix is diagonalisable, but the eigenvalues are not imaginary: $-1 \pm i$.

(79) This is Bianchi VIII $(\cong \mathfrak{s l}(2, \mathbb{R}))$ which has a faithful two-dimensional representation

$$
\rho(X)=\left(\begin{array}{cc}
x & y \\
z & -x
\end{array}\right) .
$$

Here $B=\boldsymbol{e}_{1}+\boldsymbol{e}_{3}$, which has matrix

$$
\rho(B)=\left(\begin{array}{cc}
1 & 0 \\
1 & -1
\end{array}\right),
$$

whose eigenvalues are real: \pm 1 .

In summary, after this discussion we are left with the carrollian Lie pairs: $14,16_{\varepsilon}$ and 17 in $D \geq 3$ and $40,41,42$ and 45 in $D=2$.

\subsubsection{The carrollian light cone}

It is easy to show that Lie pairs 17 and 45 are geometrically realisable. We can treat them together and, in fact, the argument works also to give an alternative proof of geometric realisability for the $D=1$ Lie pair 79 . These Lie pairs are all of the form $(\mathfrak{s o}(D+1,1), \mathfrak{h})$ where, as we now show, $\mathfrak{h}$ is the subalgebra of $\mathfrak{s o}(D+1,1)$ corresponding to the stabiliser of a null vector in the lorentzian vector space $\mathbb{R}^{D+1,1}$. Indeed let $e_{\mu}=\left\{e_{a}, e_{+}, e_{-}\right\}$be a Witt basis for $\mathbb{R}^{D+1,1}$ where the lorentzian inner product $\eta_{\mu \nu}:=\eta\left(e_{\mu}, e_{\nu}\right)$ is given by $\eta_{a b}=\delta_{a b}$ and $\eta_{+-}=1$. Then $\mathfrak{s o}(D+1,1)$ has generators $J_{\mu \nu}$ with Lie brackets:

$$
\left[J_{\mu \nu}, J_{\rho \sigma}\right]=\eta_{\nu \rho} J_{\mu \sigma}-\eta_{\mu \rho} J_{\nu \sigma}-\eta_{\nu \sigma} J_{\mu \rho}+\eta_{\mu \sigma} J_{\nu \rho}
$$

Letting $\mu=(a,+,-)$ and decomposing $J_{\mu \nu}$ into $\left\{J_{a b}, J_{a+}, J_{a-}, J_{+-}\right\}$, we obtain the following nonzero brackets:

$$
\begin{aligned}
{\left[J_{a b}, J_{c d}\right] } & =\delta_{b c} J_{a d}-\delta_{a c} J_{b d}-\delta_{b d} J_{a c}+\delta_{a d} J_{b c} \\
{\left[J_{a b}, J_{c+}\right] } & =\delta_{b c} J_{a+}-\delta_{a c} J_{b+} \\
{\left[J_{a b}, J_{c-}\right] } & =\delta_{b c} J_{a-}-\delta_{a c} J_{b-} \\
{\left[J_{+-}, J_{a+}\right] } & =J_{a+} \\
{\left[J_{+-}, J_{a-}\right] } & =-J_{a-} \\
{\left[J_{a+}, J_{b-}\right] } & =-J_{a b}-\delta_{a b} J_{+-}
\end{aligned}
$$

from where we can identify $B_{a}=J_{a+}, P_{a}=-J_{a-}$ and $H=J_{+-}$. The Lie pairs 17,45 and 79 correspond to $(\mathfrak{s o}(D+1,1), \mathfrak{h})$ where $\mathfrak{h}$ is the span of $J_{a b}$ (if $D \geq 2$ ) and $B_{a}$. The 
key observation is that $\mathfrak{h}$ is the subalgebra which annihilates the basis vector $e_{+}$under the usual action:

$$
J_{\mu \nu} \cdot e_{\rho}=\eta_{\nu \rho} e_{\mu}-\eta_{\mu \rho} e_{\nu}
$$

The connected subgroup $\mathcal{H}$ of $\mathrm{SO}(D+1,1)$ generated by $\mathfrak{h}$ is (the identity component of) the stabiliser of $e_{+}$and hence it is a closed subgroup. Since $\mathcal{H}$ is connected, it is actually a subgroup of $\mathrm{SO}(D+1,1)_{0}$, the identity component of $\mathrm{SO}(D+1,1)$. We conclude that $\mathrm{SO}(D+1,1)_{0} / \mathcal{H}$ is thus a geometric realisation of $(\mathfrak{s o}(D+1,1), \mathfrak{h})$. Geometrically, it corresponds to the future light cone $\mathscr{L}_{+} \subset \mathbb{M}$, where $\mathbb{M}$ is $(D+2)$-dimensional Minkowski spacetime. Indeed, as shown in [39] (see also [28]), null hypersurfaces (such as the future light cone in Minkowski spacetime) are carrollian spacetimes. This idea turns out to be very fruitful in order to prove the geometric realisability of the remaining symmetric carrollian Lie pairs, as we will now see.

\subsubsection{Symmetric carrollian spacetimes}

Finally, we show that the symmetric carrollian Lie pairs 14 and $16_{\varepsilon}$ in $D \geq 3$ and 40,41 and 42 in $D=2$ are geometrically realisable. As mentioned above already, one way to do this is to construct the geometric realisations explicitly by exhibiting them as null hypersurfaces in lorentzian manifolds one dimension higher. This was done originally for the carrollian spacetime C (S13) in [39], who embedded it as a null hypersurface in Minkowski spacetime one dimension higher. In a similar way we will construct the carrollian (anti) de Sitter spacetimes dSC (S14) and AdSC (S15) as null hypersurfaces in (anti) de Sitter spacetimes one dimension higher. All we need to show is that the Lie pairs describing these null hypersurfaces are the symmetric carrollian Lie pairs $14,16_{\varepsilon}, 40,41$ and 42 .

Introducing a parameter $\varepsilon=0, \pm 1$, we define the kinematical Lie algebra $\mathfrak{k}_{\varepsilon}$ by the following Lie brackets in addition to the ones in (2.5):

$$
[H, \boldsymbol{P}]=\varepsilon \boldsymbol{B}, \quad[\boldsymbol{B}, \boldsymbol{P}]=H \quad \text { and } \quad[\boldsymbol{P}, \boldsymbol{P}]=\varepsilon \boldsymbol{J} .
$$

We shall let $\mathfrak{h}_{\varepsilon}$ denote the admissible subalgebra spanned by $\boldsymbol{J}$ and $\boldsymbol{B}$. The Lie pair $\left(\mathfrak{k}_{\varepsilon}, \mathfrak{h}_{\varepsilon}\right)$ is isomorphic to 14 and 40 when $\varepsilon=0$, to $16_{+1}$ and 41 when $\varepsilon=+1$ and to $16_{-1}$ and 42 when $\varepsilon=-1$. We will now exhibit homogeneous manifolds whose Lie pairs are isomorphic to $\left(\mathfrak{k}_{\varepsilon}, \mathfrak{h}_{\varepsilon}\right)$ for each value of $\varepsilon$.

We start with $\varepsilon=0$, which is the construction of flat carrollian space C (S13) in [39]. To this end, let $\mathbb{M}$ denote $(D+2)$-dimensional Minkowski spacetime with coordinates $x^{\mu}$ for $\mu=0,1, \ldots, D+1$ (although we will let $\downarrow$ stand for $D+1$ ) and metric

$$
d s^{2}=\eta_{\mu \nu} d x^{\mu} d x^{\mu}=-\left(d x^{0}\right)^{2}+\sum_{a=1}^{D}\left(d x^{a}\right)^{2}+\left(d x^{\natural}\right)^{2}=2 d x^{+} d x^{-}+\sum_{a=1}^{D}\left(d x^{a}\right)^{2},
$$

where $x^{ \pm}=\frac{1}{\sqrt{2}}\left(x^{\natural} \pm x^{0}\right)$. Let $\mathscr{C}_{0} \subset \mathbb{M}$ denote the null hypersurface defined by $x^{-}=0$. We claim that $\mathscr{C}_{0}$ is a geometric realisation of the Lie pair $\left(\mathfrak{k}_{0}, \mathfrak{h}_{0}\right)$.

The Poincaré Lie algebra is spanned by the following vector fields on $\mathbb{M}$ :

$$
J_{\mu \nu}=x_{\mu} \frac{\partial}{\partial x^{\nu}}-x_{\nu} \frac{\partial}{\partial x^{\mu}} \quad \text { and } \quad P_{\mu}=\frac{\partial}{\partial x^{\mu}},
$$


where $x_{\mu}=\eta_{\mu \rho} x^{\rho}$. It is a transitive Lie algebra for $\mathbb{M}$, which means that for every $p \in \mathbb{M}$, the values $J_{\mu \nu}(p)$ and $P_{\mu}(p)$ span the tangent space $T_{p} \mathbb{M}$. The subalgebra of the Poincaré algebra consisting of vector fields which are tangent to $\mathscr{C}$ is spanned by

$$
J_{a b}, \quad P_{a}, \quad B_{a}:=J_{a 0}+J_{a \natural} \quad \text { and } \quad H:=P_{0}+P_{\natural} .
$$

It is easy to see that these satisfy the Lie brackets of $\mathfrak{k}_{0}$ (the Carroll algebra) and the subalgebra $\mathfrak{h}_{0}$ which vanishes at the point $o \in \mathscr{C}_{0}$ with coordinates $x^{\mu}=0$ is the span of $J_{a b}$ and $B_{a}$. By dimension, $P_{a}$ and $H$ span the tangent space $T_{o} \mathscr{C}_{0}$ and indeed the same is true at any other point of $\mathscr{C}$ with different (but isomorphic) stabiliser subalgebra. Hence $\mathfrak{k}_{0}$ is a transitive Lie algebra for $\mathscr{C}_{0}$ with stabiliser $\mathfrak{h}_{0}$ at $o$. Therefore $\mathscr{C}_{0}$ is a geometric realisation of $\left(\mathfrak{k}_{0}, \mathfrak{h}_{0}\right)$.

Now let us consider $\varepsilon=-1$ and let $\mathbb{M}$ now stand for Minkowski spacetime of dimension $D+3$, with coordinates $x^{\mu}$ with $\mu=0,1, \ldots, D+2$ and metric

$$
d s^{2}=\eta_{\mu \nu} d x^{\mu} d x^{\nu}=-\left(d x^{0}\right)^{2}+\sum_{A=1}^{D+2}\left(d x^{A}\right)^{2} .
$$

Let $\mathscr{Q}_{-}$denote the quadric defined by

$$
\eta_{\mu \nu} x^{\mu} x^{\nu}=R^{2}
$$

for some (fixed) $R>0$. Its universal cover (with the induced metric) is de Sitter spacetime in dimension $D+2$. The Lorentz Lie algebra, spanned by

$$
J_{\mu \nu}=x_{\mu} \frac{\partial}{\partial x^{\nu}}-x_{\nu} \frac{\partial}{\partial x^{\mu}}
$$

with $x_{\mu}=\eta_{\mu \rho} x^{\rho}$, is a transitive Lie algebra for $\mathscr{Q}_{-}$and isomorphic to $\mathfrak{s o}(D+2,1)$. Let $\mathscr{N} \subset \mathbb{M}$ denote the null hypersurface defined by the equation $x^{0}=x^{D+2}$ and let $\mathscr{C}_{-}=$ $\mathscr{Q}_{-} \cap \mathscr{N}$. This is defined by the following two equations:

$$
x^{0}=x^{D+2} \quad \text { and } \quad \sum_{i=1}^{D+1}\left(x^{i}\right)^{2}=R^{2},
$$

which shows that $\mathscr{C}_{-}$is diffeomorphic to $\mathbb{R} \times \mathbb{S}^{D}$ and hence is simply-connected for $D \geq 2$. We claim that $\mathscr{C}_{-}$is a geometric realisation for the Lie pair $\left(\mathfrak{k}_{-}, \mathfrak{h}_{-}\right)$. We will show this by determining the subalgebra of the Lorentz Lie algebra consisting of vector fields tangent to $\mathscr{C}_{-}$, which by the same argument as in the previous case is transitive on $\mathscr{C}_{-}$. We will show that this subalgebra is isomorphic to $\mathfrak{k}_{-}$and that the stabiliser at a suitably chosen point $o \in \mathscr{C}_{-}$is isomorphic to $\mathfrak{h}_{-}$. The following Lorentz generators are tangent to $\mathscr{C}_{-}$:

$$
J_{i j} \quad \text { and } \quad V_{i}:=J_{i 0}+J_{i, D+2},
$$

for all $i=1, \ldots, D+1$. This Lie algebra is isomorphic to the euclidean algebra in dimension $D+1$, with nonzero Lie brackets

$$
\begin{aligned}
{\left[J_{i j}, J_{k \ell}\right] } & =\delta_{j k} J_{i \ell}-\delta_{i k} J_{j \ell}-\delta_{j \ell} J_{i k}+\delta_{i \ell} J_{j k} \\
{\left[J_{i j}, V_{k}\right] } & =\delta_{j k} V_{i}-\delta_{i k} V_{j} .
\end{aligned}
$$


Let $a=1, \ldots, D$ and let $\natural=D+1$. Then these generators break up as

$$
J_{a b}, \quad P_{a}:=J_{a \natural}, \quad B_{a}:=V_{a} \quad \text { and } \quad H:=V_{\natural},
$$

which obey

$$
\left[H, P_{a}\right]=-B_{a}, \quad\left[B_{a}, P_{b}\right]=\delta_{a b} H \quad \text { and } \quad\left[P_{a}, P_{b}\right]=-J_{a b},
$$

apart from (2.5). We see that this Lie algebra is isomorphic to $\mathfrak{k}_{-}$. Now let $o \in \mathscr{C}_{-}$denote the point with coordinates $x^{a}=x^{0}=x^{D+2}=0$ and $x^{\natural}=R$. Then the vector fields which vanish at $o$ are the span of $J_{a b}$ and $B_{a}$, which is isomorphic to $\mathfrak{h}_{-}$. Therefore $\mathscr{C}_{-}$is a geometric realisation of $\left(\mathfrak{k}_{-}, \mathfrak{h}_{-}\right)$.

Finally, we consider the case $\varepsilon=+1$. Now $\widetilde{\mathbb{E}}$ is pseudo-euclidean space with signature $(D+1,2)$ with coordinates $x^{\mu}$ for $\mu=0,1, \ldots, D+2$ and metric

$$
d s^{2}=\eta_{\mu \nu} d x^{\mu} d x^{\nu}=-\left(d x^{0}\right)^{2}+\sum_{i=1}^{D+1}\left(d x^{i}\right)^{2}-\left(d x^{D+2}\right)^{2}
$$

metric as above. Now we fix $R>0$ and let $\mathscr{Q}_{+} \subset \widetilde{\mathbb{E}}$ denote the quadric defined by the equation

$$
\eta_{\mu \nu} x^{\mu} x^{\nu}=-R^{2} .
$$

Its universal cover (with the induced metric) is anti de Sitter spacetime in dimension $D+2$. The Lie algebra spanned by the vector fields

$$
J_{\mu \nu}=x_{\mu} \frac{\partial}{\partial x^{\nu}}-x_{\nu} \frac{\partial}{\partial x^{\mu}}
$$

with $x_{\mu}=\eta_{\mu \rho} x^{\rho}$, is a transitive Lie algebra for $\mathscr{Q}_{+}$and isomorphic to $\mathfrak{s o}(D+1,2)$. Let $\mathscr{N} \subset \widetilde{\mathbb{E}}$ denote now the null hypersurface with equation $x^{D+1}=x^{D+2}$. The intersection $\mathscr{Q}_{+} \cap \mathscr{N}$ is described by the two equations:

$$
x^{D+1}=x^{D+2} \quad \text { and } \quad\left(x^{0}\right)^{2}=R^{2}+\sum_{a=1}^{D}\left(x^{a}\right)^{2},
$$

which has two connected components determined by the sign of $x^{0}$, which is never zero. Let $\mathscr{C}_{+}$denote the component where $x^{0}>0$. The following vector fields generate the subalgebra of the span of the $J_{\mu \nu}$ which are tangent to $\mathscr{C}_{+}$:

$$
J_{a b}, \quad P_{a}:=-J_{0 a}, \quad B_{a}:=J_{a, D+1}+J_{a, D+2} \quad \text { and } \quad H:=J_{0, D+1}+J_{0, D+2},
$$

for $a=1, \ldots, D$. In addition to $(2.5)$, they have the following nonzero Lie brackets

$$
\left[H, P_{a}\right]=B_{a} \quad\left[B_{a}, P_{b}\right]=\delta_{a b} H \quad \text { and } \quad\left[P_{a}, P_{b}\right]=J_{a b},
$$

which is isomorphic to $\mathfrak{k}_{+}$. The stabiliser subalgebra at the point $o \in \mathscr{C}_{+}$with coordinates $x^{a}=x^{D+1}=x^{D+2}=0$ and $x^{0}=R$, is the span of $J_{a b}$ and $B_{a}$, so isomorphic to $\mathfrak{h}_{+}$. As before, dimension says that $\mathfrak{k}_{+}$is transitive at $o \in \mathscr{C}_{+}$with stabiliser $\mathfrak{h}_{+}$and also at other points with isomorphic stabilisers. Therefore $\mathscr{C}_{+}$is a geometric realisation of $\left(\mathfrak{k}_{+}, \mathfrak{h}_{+}\right)$.

It is worth remarking that in all the homogeneous carrollian spacetimes discussed here, the boost generators can be interpreted as null rotations in the ambient Minkowski or pseudo-euclidean spaces. 


\section{Limits between homogeneous spacetimes}

In the previous two sections we have classified the simply-connected homogeneous spacetimes. This provides the objects in figure 3, which also contains arrows between the spacetimes. These arrows are explained by limits between spacetimes and in this section we will discuss these limits and in this way explain figure 3. We will also explain how the picture gets modified in $D \leq 2$ and explain figures 4 and 5 .

In the infinitesimal description of the homogeneous spacetimes in terms of Lie pairs, most (but not all) limits between homogeneous spacetimes manifest themselves as contractions of the underlying kinematical Lie algebras.

\subsection{Contractions}

Recall that a (finite-dimensional, real) Lie algebra consists of a vector space $V$, together with a linear map $\varphi: \Lambda^{2} V \rightarrow V$ satisfying the Jacobi identity. The Jacobi identity defines an algebraic variety $\mathscr{J} \subset \Lambda^{2} V^{*} \otimes V$, every point of which is a Lie algebra structure on $V$. The general linear group $\mathrm{GL}(V)$ acts on $V$ and hence also on the vector space $\Lambda^{2} V^{*} \otimes V$ and since the action is tensorial it preserves the variety $\mathscr{J}$. If $\varphi \in \mathscr{J}$ defines a Lie algebra $\mathfrak{g}$ and $g \in \mathrm{GL}(V)$, then $g \cdot \varphi \in \mathscr{J}$ and, by definition, the Lie algebra it defines is isomorphic to $\mathfrak{g}$. Indeed, the $\mathrm{GL}(V)$ orbit of $\varphi$ consists of all Lie algebras on $V$ which are isomorphic to $\mathfrak{g}$. This orbit may not be closed relative to the induced topology on $\mathscr{J}$. The closure of the orbit may contain Lie algebras which are not isomorphic to $\mathfrak{g}$ : they are said to be "degenerations" of $\mathfrak{g}$. A special class of degenerations are the contractions of $\mathfrak{g}$, which are limit points of curves in the $\mathrm{GL}(V)$-orbit of $\varphi$. More precisely, let $t \in(0,1]$ and let $g_{t}$ be a continuous curve in $\mathrm{GL}(V)$ with $g_{1}$ the identity. Define $\varphi_{t}:=g_{t} \cdot \varphi$. Explicitly, the Lie bracket $[-,-]_{t}$ associated to $\varphi_{t}$ is given by

$$
[X, Y]_{t}=g_{t} \cdot\left[g_{t}^{-1} \cdot X, g_{t}^{-1} \cdot Y\right] .
$$

Then $\varphi_{1}=\varphi$ and for every $t \in(0,1], \varphi_{t}$ defines a Lie algebra on $V$ isomorphic to $\mathfrak{g}$. By continuity, if the limit $\varphi_{0}:=\lim _{t \rightarrow 0} \varphi_{t}$ exists, it defines a Lie algebra, but since the linear transformation $g_{0}:=\lim _{t \rightarrow 0} g_{t}$ of $V$ (even if it exists) need not be invertible, the Lie algebra defined by $\varphi_{0}$ need not be isomorphic to $\mathfrak{g}$. It is, however, by definition a contraction of $\mathfrak{g}$.

We will now explicitly exhibit contractions between kinematical Lie algebras which induce the limits between the $(D+1)$-dimensional homogeneous spaces in figure 3 for $D \geq 3$. We will also explain other non-contraction limits in that figure, as well as in $D \leq 2$.

\section{$5.2 \quad D \geq 3$}

We will start with the lorentzian and riemannian space forms with nonzero curvature, whose kinematical Lie algebras are the (semi)simple Lie algebras: $\mathfrak{s o}(D+1,1)$ for de Sitter spacetime (S2) and hyperbolic space $(\mathrm{S} 6), \mathfrak{s o}(D, 2)$ for anti de Sitter spacetime (S3) and $\mathfrak{s o}(D+2)$ for the round sphere (S5).

Let $\mathbb{R}^{D+2}$ have basis $e_{\mu}=\left(e_{0}, e_{a}, e_{\natural}\right)$, with $a=1, \ldots, D$ and let $\eta$ denote the inner product with coefficients $\eta_{\mu \nu}=\eta\left(e_{\mu}, e_{\nu}\right)$ given by $\eta_{a b}=\delta_{a b}$ and all other components zero 
except for $\eta_{00}$ and $\eta_{\text {耴. }}$ The generators of $\mathfrak{s o}\left(\mathbb{R}^{D+2}, \eta\right)$ are $J_{\mu \nu}=\left\{J_{a b}, B_{a}:=J_{0 a}, P_{a}:=\right.$ $\left.J_{a \natural}, H:=J_{0 \natural}\right\}$ subject to the following Lie brackets

$$
\begin{aligned}
& {\left[J_{a b}, J_{c d}\right]=\delta_{b c} J_{a d}-\delta_{a c} J_{b d}-\delta_{b d} J_{a c}+\delta_{a d} J_{b c} \quad\left[H, P_{a}\right]=-\eta_{\text {七中 }} B_{a}} \\
& {\left[J_{a b}, B_{c}\right]=\delta_{b c} B_{a}-\delta_{a c} B_{b} \quad\left[B_{a}, B_{b}\right]=-\eta_{00} J_{a b}} \\
& {\left[J_{a b}, P_{c}\right]=\delta_{b c} P_{a}-\delta_{a c} P_{b} \quad\left[P_{a}, P_{b}\right]=-\eta_{\text {घh }} J_{a b}} \\
& {\left[H, B_{a}\right]=\eta_{00} P_{a} \quad\left[B_{a}, P_{b}\right]=\delta_{a b} H .}
\end{aligned}
$$

The first three brackets are the standard kinematical Lie brackets, so we will focus attention on the remaining brackets and we will change to shorthand notation where the $\mathfrak{s o}(D)$ indices are implicit.

Let us consider a three-parameter $(\kappa, c, \tau)$ family of linear transformations $g_{\kappa, c, \tau}$ defined on generators by

$$
g_{\kappa, c, \tau} \cdot \boldsymbol{J}=\boldsymbol{J}, \quad g_{\kappa, c, \tau} \cdot \boldsymbol{B}=\frac{\tau}{c} \boldsymbol{B}, \quad g_{\kappa, c, \tau} \cdot \boldsymbol{P}=\frac{\kappa}{c} \boldsymbol{P} \quad \text { and } \quad g_{\kappa, c, \tau} \cdot H=\tau \kappa H .
$$

The transformed Lie brackets are such that the common kinematical Lie brackets involving $\boldsymbol{J}$ are unchanged and the remaining brackets are

$$
\begin{aligned}
& {[H, \boldsymbol{B}]=\tau^{2} \eta_{00} \boldsymbol{P}} \\
& {[H, \boldsymbol{P}]=-\kappa^{2} \eta_{\text {七七 }} \boldsymbol{B}} \\
& {[\boldsymbol{B}, \boldsymbol{B}]=-\left(\frac{\tau}{c}\right)^{2} \eta_{00} \boldsymbol{J}} \\
& {[\boldsymbol{P}, \boldsymbol{P}]=-\left(\frac{\kappa}{c}\right)^{2} \eta_{\text {Łฝ }} \boldsymbol{J}} \\
& {[\boldsymbol{B}, \boldsymbol{P}]=\left(\frac{1}{c}\right)^{2} H .}
\end{aligned}
$$

The flat limit corresponds to taking $\kappa \rightarrow 0$, the non-relativistic limit to $c \rightarrow \infty$ and the ultra-relativistic limit to $\tau \rightarrow 0$. We may take any two of the three limits or, indeed, all limits at once; although whenever we combine a non-relativistic limit with an ultrarelativistic limit we arrive at a non-effective Lie pair reducing to the aristotelian static spacetime (A21), denoted $\mathrm{S}$ in figure 3.

Let us first take the flat limit $\kappa \rightarrow 0$ of the Lie brackets in (5.4) to arrive at

$$
\begin{aligned}
& {[H, \boldsymbol{B}]=\tau^{2} \eta_{00} \boldsymbol{P}} \\
& {[\boldsymbol{B}, \boldsymbol{B}]=-\left(\frac{\tau}{c}\right)^{2} \eta_{00} \boldsymbol{J}} \\
& {[\boldsymbol{B}, \boldsymbol{P}]=\left(\frac{1}{c}\right)^{2} H}
\end{aligned}
$$

For $\frac{\tau}{c} \neq 0$, this is either the Poincaré Lie algebra for $\eta_{00}=-1$ or the euclidean Lie algebra for $\eta_{00}=1$. The corresponding Lie pairs are those of Minkowski spacetime (S1) and euclidean space (S4). In figure 3 only $\eta_{00}=-1$ is considered and these limits explain the arrows AdS $\rightarrow \mathbb{M}$ and $d S \rightarrow \mathbb{M}$, but in figure 2 this also explains the arrows $\mathbb{H} \rightarrow \mathbb{E}$ and 
$\mathbb{S} \rightarrow \mathbb{E}$. We may now take the non-relativistic limit $c \rightarrow \infty$ to arrive at the galilean algebra (after rescaling $H$ by $-1 /\left(\eta_{00} \tau^{2}\right)$ ),

$$
[H, \boldsymbol{B}]=-\boldsymbol{P},
$$

or alternatively the ultra-relativistic limit $\tau \rightarrow 0$ to arrive at the Carroll algebra (after setting $c=1)$ :

$$
[\boldsymbol{B}, \boldsymbol{P}]=H .
$$

The corresponding Lie pairs are the galilean (S7) and carrollian (S13) spacetimes. This explains the arrows $\mathbb{M} \rightarrow \mathrm{G}$ and $\mathbb{M} \rightarrow \mathrm{C}$ in figure 3 and the arrows $\mathbb{E} \rightarrow \mathrm{G}$ and $\mathbb{E} \rightarrow \mathrm{C}$ in figure 2.

Taking now the non-relativistic limit of the Lie brackets in (5.4), we have

$$
\begin{aligned}
& {[H, \boldsymbol{B}]=\tau^{2} \eta_{00} \boldsymbol{P}} \\
& {[H, \boldsymbol{P}]=-\kappa^{2} \eta_{\text {Łฝ }} \boldsymbol{B} .}
\end{aligned}
$$

For $\tau \kappa \neq 0$, we obtain the Lie pairs corresponding to the galilean de Sitter spacetime (S8), if $\eta_{\natural \natural} \eta_{00}=-1$, or the galilean anti de Sitter spacetime (S10), if $\eta_{\sharp \downarrow} \eta_{00}=1$, thus explaining the arrows dS $\rightarrow$ dSG and AdS $\rightarrow$ AdSG in figure 3 and also the arrows $\mathbb{H} \rightarrow$ dSG and $\mathbb{S} \rightarrow$ AdSG in figure 2. If we then take the flat limit $\kappa \rightarrow 0$, we obtain the galilean spacetime, thus explaining the arrows dSG $\rightarrow$ G and AdSG $\rightarrow$ G. If instead we take the ultra-relativistic limit we obtain a non-effective Lie pair reducing to the aristotelian static spacetime (A21).

Finally, let us start by taking the ultra-relativistic limit $(\tau \rightarrow 0)$ in the brackets (5.4), to arrive at

$$
\begin{aligned}
& {[H, \boldsymbol{P}]=-\kappa^{2} \eta_{\text {耴 }} \boldsymbol{B}} \\
& {[\boldsymbol{P}, \boldsymbol{P}]=-\left(\frac{\kappa}{c}\right)^{2} \eta_{\text {Ł }} \boldsymbol{J}} \\
& {[\boldsymbol{B}, \boldsymbol{P}]=\left(\frac{1}{c}\right)^{2} H .}
\end{aligned}
$$

If $\frac{\kappa}{c} \neq 0$, we obtain either the carrollian de Sitter spacetime (S14) if $\eta_{\text {如 }}=1$ or the carrollian anti de Sitter spacetime (S15) if $\eta_{\text {七 }}=-1$. This explains the arrows dS $\rightarrow$ dSC and AdS $\rightarrow$ AdSC in figure 3 and the arrows $\mathbb{H} \rightarrow$ AdSC and $\mathbb{S} \rightarrow$ dSC in figure 2 . If we now take the flat limit we arrive at the carrollian spacetime, which explains the arrows dSC $\rightarrow \mathrm{C}$ and AdSC $\rightarrow$ C. If instead we take the non-relativistic limit, we arrive at a non-effective Lie pair reducing to the aristotelian static spacetime (A21).

Of all the arrows to the aristotelian static spacetime, only $\mathrm{G} \rightarrow \mathrm{S}$ and $\mathrm{C} \rightarrow \mathrm{S}$ are shown explicitly in figure 3. Taking any two limits in the brackets (5.4), the resulting Lie algebra does not depend on the order in which we take the limits. This means that the arrows in (5.4) "commute" and thus, for instance, that the arrow (not shown) dSC $\rightarrow \mathrm{S}$ is to be understood as the composition of the arrows (shown) dSC $\rightarrow \mathrm{C} \rightarrow \mathrm{S}$. Similarly, the arrows (not shown) AdSC $\rightarrow \mathrm{S}$, dSG $\rightarrow \mathrm{S}$ and AdSG $\rightarrow \mathrm{S}$ can be understood as compositions of arrows which are shown: AdSC $\rightarrow \mathrm{C} \rightarrow \mathrm{S}, \mathrm{dSG} \rightarrow \mathrm{G} \rightarrow \mathrm{S}$ and AdSG $\rightarrow \mathrm{G} \rightarrow \mathrm{S}$, respectively. 
We have so far explained the limits in figure 3 (or even figure 2) corresponding to the known symmetric spacetimes and it now remains to explain the limits from the new spacetimes in our classification.

\subsection{1 $\quad \mathrm{AdSG}_{\chi} \rightarrow \mathrm{G}$}

Let $t \in(0,1]$ and let $g_{t}$ be defined by

$$
g_{t} \cdot \boldsymbol{J}=\boldsymbol{J}, \quad g_{t} \cdot \boldsymbol{B}=\boldsymbol{B}, \quad g_{t} \cdot \boldsymbol{P}=t \boldsymbol{P} \quad \text { and } \quad g_{t} \cdot H=t H .
$$

The new brackets are now

$$
[H, \boldsymbol{B}]=-\boldsymbol{P} \quad \text { and } \quad[H, \boldsymbol{P}]=t^{2}\left(1+\chi^{2}\right) \boldsymbol{B}+2 t \chi \boldsymbol{P},
$$

so that taking the limit $t \rightarrow 0$, gives the galilean algebra $[H, \boldsymbol{B}]=-\boldsymbol{P}$.

\subsection{2 $\mathrm{dSG}_{\gamma} \rightarrow \mathrm{G}$}

This is just like the previous case. Under the same $g_{t}$ as before, the new brackets are now

$$
[H, \boldsymbol{B}]=-\boldsymbol{P} \quad \text { and } \quad[H, \boldsymbol{P}]=t^{2} \gamma \boldsymbol{B}+t(1+\gamma) \boldsymbol{P},
$$

so that taking the limit $t \rightarrow 0$, gives the galilean algebra.

\subsubsection{LC $\rightarrow$ C}

Taking $g_{t}$ as in the previous two cases, the brackets become

$$
[H, \boldsymbol{B}]=t \boldsymbol{B}, \quad[H, \boldsymbol{P}]=t \boldsymbol{P} \quad \text { and } \quad[\boldsymbol{B}, \boldsymbol{P}]=H+t \boldsymbol{J} .
$$

Taking the limit $t \rightarrow 0$ we recover the Carroll algebra $[\boldsymbol{B}, \boldsymbol{P}]=H$.

\subsubsection{LC $\rightarrow$ TS}

Let $t \in(0,1]$ and let $g_{t}$ be defined by

$$
g_{t} \cdot \boldsymbol{J}=\boldsymbol{J}, \quad g_{t} \cdot \boldsymbol{B}=\boldsymbol{B}, \quad g_{t} \boldsymbol{P}=t \boldsymbol{P} \quad \text { and } \quad g_{t} \cdot H=H,
$$

so that the brackets become

$$
[H, \boldsymbol{B}]=\boldsymbol{B}, \quad[H, \boldsymbol{P}]=\boldsymbol{P} \quad \text { and } \quad[\boldsymbol{B}, \boldsymbol{P}]=t H+t \boldsymbol{J} .
$$

Taking the limit $t \rightarrow 0$ gives

$$
[H, \boldsymbol{B}]=\boldsymbol{B} \quad \text { and } \quad[H, \boldsymbol{P}]=\boldsymbol{P} .
$$

The resulting Lie pair is not effective because the span of the $B_{a}$ is an ideal. Quotienting by this ideal gives the aristotelian Lie algebra defined by $[H, \boldsymbol{P}]=\boldsymbol{P}$, whose associated spacetime is the torsional static spacetime A22. 


\subsubsection{TS $\rightarrow$ S}

Let $t \in(0,1]$ and let $g_{t} \cdot H=t H$ and $g_{t} \cdot P=P$. The new bracket is $[H, P]=t P$, which vanishes in the limit $t \rightarrow 0$.

\subsubsection{A non-contracting limit}

Finally, we discuss a limit which does not come from a contraction of Lie algebras. The Lie algebra of $\mathrm{AdSG}_{\chi}$ depends on a parameter $\chi \geq 0$ and this parameter determines the isomorphism class of the Lie algebra. A natural question is what spacetime corresponds to $A d S G_{\chi}$ in the limit $\chi \rightarrow \infty$. The answer turns out to be that $\lim _{\chi \rightarrow \infty} \mathrm{AdSG}_{\chi}=d \mathrm{dSG}_{1}$. To see this we start with the Lie algebra corresponding to $\mathrm{AdSG}_{\chi}$

$$
[H, \boldsymbol{B}]=-\boldsymbol{P} \quad \text { and } \quad[H, \boldsymbol{P}]=\left(1+\chi^{2}\right) \boldsymbol{B}+2 \chi \boldsymbol{P}
$$

and we change basis to

$$
H^{\prime}=\chi^{-1} H \quad \boldsymbol{B}^{\prime}=\boldsymbol{B} \quad \text { and } \quad \boldsymbol{P}^{\prime}=\chi^{-1} \boldsymbol{P} .
$$

This is a vector space isomorphism for any $\chi>0$, but becomes singular in the limit $\chi \rightarrow \infty$. In this sense this is reminiscent of a contraction, but it is not a contraction since we are changing the isomorphism type of the Lie algebra as we change $\chi$. In the new basis,

$$
\left[H^{\prime}, \boldsymbol{B}^{\prime}\right]=-\boldsymbol{P}^{\prime} \quad \text { and } \quad\left[H^{\prime}, \boldsymbol{P}^{\prime}\right]=\left(1+\chi^{-2}\right) \boldsymbol{B}^{\prime}+2 \boldsymbol{P}^{\prime}
$$

and now taking $\chi \rightarrow \infty$ we arrive at

$$
\left[H^{\prime}, \boldsymbol{B}^{\prime}\right]=-\boldsymbol{P}^{\prime} \quad \text { and } \quad\left[H^{\prime}, \boldsymbol{P}^{\prime}\right]=\boldsymbol{B}^{\prime}+2 \boldsymbol{P}^{\prime},
$$

which is the Lie algebra corresponding to $\mathrm{dSG}_{1}$.

\section{$5.3 \quad D=2$}

In $D=2$ there is an additional two-parameter family of spacetimes not present in $D \geq 3$ : namely, spacetime $\mathrm{S} 12_{\gamma, \chi}$, for $\gamma \in[-1,1)$ and $\chi>0$. The Lie brackets are given by (2.5) and in addition

$$
[H, \boldsymbol{B}]=-\boldsymbol{P} \quad \text { and } \quad[H, \boldsymbol{P}]=(1+\gamma) \boldsymbol{P}-\chi \widetilde{\boldsymbol{P}}+\gamma \boldsymbol{B}-\chi \widetilde{\boldsymbol{B}},
$$

or in complex form

$$
[H, \boldsymbol{B}]=-\boldsymbol{P} \quad \text { and } \quad[H, \boldsymbol{P}]=(1+z) \boldsymbol{P}+z \boldsymbol{B},
$$

where $z=\gamma+i \chi \in \mathbb{C}$ lies in the infinite vertical strip in the upper-half plane defined by $-1 \leq \operatorname{Re} z<1$.

Parenthetically, let us mention that for $z=-1+i \chi$, the complexified Lie pair for spacetime $\mathrm{S}_{-1, \chi}$ is isomorphic to the complexification of the Lie pair for dSG. This can be seen by the following complex change of basis:

$$
H^{\prime}=\frac{2}{2+i \chi}\left(H+\frac{\chi}{2} J\right) \quad \boldsymbol{B}^{\prime}=\boldsymbol{B} \quad \text { and } \quad \boldsymbol{P}^{\prime}=\frac{2}{2+i \chi}\left(\boldsymbol{P}+i \frac{\chi}{2} \boldsymbol{B}\right)
$$




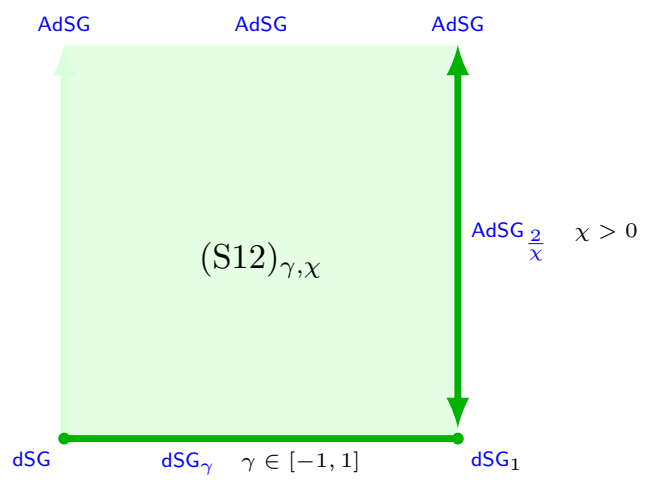

Figure 6. Parameter space for some $D=2$ spacetimes.

so that

$$
\left[H^{\prime}, \boldsymbol{B}^{\prime}\right]=-\boldsymbol{P}^{\prime} \quad \text { and } \quad\left[H^{\prime}, \boldsymbol{P}^{\prime}\right]=-\boldsymbol{B}^{\prime} .
$$

This provides an example of a finite-dimensional complex Lie algebra having a continuum of non-isomorphic real forms.

The region where $z$ lives has two additional boundaries: $z=\gamma \in[-1,1]$ and $z=$ $1+i \chi$ with $\chi \geq 0$. The horizontal boundary $z=\gamma \in[-1,1]$ corresponds to $\chi=0$ in equation (5.21):

$$
[H, \boldsymbol{B}]=-\boldsymbol{P} \quad \text { and } \quad[H, \boldsymbol{P}]=\gamma \boldsymbol{B}+(1+\gamma) \boldsymbol{P},
$$

which corresponds to $\mathrm{dSG}_{\gamma}$.

Let us change basis (for $\chi>0)$ from $(H, \boldsymbol{B}, \boldsymbol{P})$ to $\left(H^{\prime}:=\frac{2}{\chi} H+J, \boldsymbol{B}^{\prime}:=\boldsymbol{B}, \boldsymbol{P}^{\prime}:=\right.$ $\left.i \boldsymbol{B}+\frac{2}{\chi} \boldsymbol{P}\right)$ in such a way that the Lie brackets become

$$
\left[H^{\prime}, \boldsymbol{B}^{\prime}\right]=-\boldsymbol{P}^{\prime} \quad \text { and } \quad\left[H^{\prime}, \boldsymbol{P}^{\prime}\right]=\frac{2(1+\gamma)}{\chi} \boldsymbol{P}^{\prime}+\left(1+\frac{4 \gamma^{2}}{\chi^{2}}+\frac{2(1-\gamma) i}{\chi}\right) \boldsymbol{B}^{\prime} .
$$

When $\gamma=1$, this corresponds to $\mathrm{AdSG}_{2 / \chi}$. Now let us consider the (singular) limit $\chi \rightarrow \infty$, so that the Lie brackets become

$$
\left[H^{\prime}, \boldsymbol{B}^{\prime}\right]=-\boldsymbol{P}^{\prime} \quad \text { and } \quad\left[H^{\prime}, \boldsymbol{P}^{\prime}\right]=\boldsymbol{B}^{\prime},
$$

which we recognise as AdSG.

The picture resulting from this discussion is illustrated in figure 6 . This shows that in $D=2$, spacetime $\mathrm{S} 12_{\gamma, \chi}$ interpolates between the one-dimensional continua of torsional galilean de Sitter and anti de Sitter spacetimes. Figure 4 shows how to insert this figure into figure 3 .

\section{$5.4 D=1$}

Now the spacetimes are two-dimensional. It is then mostly a matter of convention what we call space and what we call time. This manifests itself in some accidental isomorphisms between spacetimes. For example, de Sitter and anti de Sitter spacetimes are isomorphic 
as homogeneous spaces of $\mathrm{SO}(2,1) \cong \mathrm{SO}(1,2)$. At first one might be surprised at this statement since after all de Sitter space has positive scalar curvature, whereas anti de Sitter space has negative scalar curvature and surely they are geometrically distinguishable. This is perhaps a good place to point out that scalar curvature is not an invariant of a homogeneous space, but rather of the homogeneous space together with the choice of an invariant metric. In $(\mathrm{A}) \mathrm{dS}$ there is a one-parameter family of invariant metrics, labelled by the radius of curvature, all sharing the same connection and curvature: after all, the Levi-Civita connection and hence the Riemann curvature are homothety invariant. Even the Ricci tensor is homothety invariant and it is only the Ricci scalar, which involves tracing with the metric, that distinguishes between the (A)dS spacetimes with different radii of curvature. In two dimensions, we have the possibility of exchanging space and time, which results in multiplying the metric by -1 , which is formally a homothety, so that the Riemann and Ricci tensors are unchanged. It is only when we calculate the Ricci scalar that we see the effect of this homothety: namely, changing the sign.

There are other accidental isomorphisms coming from exchanging space and time, e.g., galilean and carrollian spacetimes are isomorphic. This is clear from figure 1 since exchanging space and time rotates the light cones by 90 degrees. Similarly, carrollian dS and galilean AdS spacetimes are isomorphic, as are carrollian AdS and galilean dS spacetimes: the result of both changing the sign of the curvature and rotating the light cone by 90 degrees.

In addition to these identifications, there are additional homogeneous spacetimes which are unique to two dimensions: namely, S17, S18, S19 $\chi>0$ and $\mathrm{S} 20_{\chi>0}$ and which admit none of the low-rank invariant structures we have been focussing on. We describe two kinds of limits between these exotic spacetimes: limits which manifest themselves infinitesimally as contractions of the relevant Bianchi Lie algebra as well as limits which are not of this type. The contractions between the Bianchi Lie algebras have been determined in [53]. All Lie algebras contract to the abelian Lie algebra (here, Bianchi I), and we will not mention these contractions explicitly. The resulting spacetimes and their limits are depicted in figure 5 .

\subsection{1 $\mathrm{S} 17 \rightarrow \mathrm{S} 18$ and $\mathrm{S} 17 \rightarrow \mathrm{S} 7 / \mathrm{S} 13$}

The Lie algebra associated to S17 is Bianchi IV, which can contract to Bianchi II and Bianchi V [53]. Indeed, both contractions arise from limits of spacetime S17: a limit to galilean/carrollian spacetime and a limit to S18.

The Lie algebra has brackets $[H, B]=-P$ and $[B, P]=-H-2 P$. This is an extension by $B$ of the abelian Lie algebra spanned by $H$ and $P$. The action of $B$ is via a nondiagonalisable endomorphism with one eigenvector with eigenvalue -1 . This means that we can change basis in the span of $H$ and $P$ so that relative to the new basis $H^{\prime}, P^{\prime}$,

$$
\left[B, H^{\prime}\right]=-H^{\prime} \quad \text { and } \quad\left[B, P^{\prime}\right]=-P^{\prime}+H^{\prime} .
$$

If we now introduce a parameter $t \in(0,1]$ and a one-parameter family $g_{t}$ of invertible endomorphisms defined by

$$
g_{t} \cdot H^{\prime}=H^{\prime}, \quad g_{t} \cdot B=B \quad \text { and } \quad g_{t} \cdot P^{\prime}=t P^{\prime},
$$


the brackets become

$$
\left[B, H^{\prime}\right]_{t}=-H^{\prime} \quad \text { and } \quad\left[B, P^{\prime}\right]=-P^{\prime}+t H^{\prime}
$$

so that the limit $t \rightarrow 0$ recovers the Lie algebra corresponding to spacetime S18.

If instead we define $g_{t}$ by

$$
g_{t} \cdot H^{\prime}=t H^{\prime}, \quad g_{t} \cdot B=t B \quad \text { and } \quad g_{t} \cdot P^{\prime}=P^{\prime},
$$

the brackets become

$$
\left[B, H^{\prime}\right]_{t}=-t H^{\prime} \quad \text { and } \quad\left[B, P^{\prime}\right]=-t P^{\prime}+H^{\prime},
$$

so that the limit $t \rightarrow 0$ recovers the Lie algebra corresponding to spacetime $\mathrm{S} 7 / \mathrm{S} 13$ after exchanging $H^{\prime}$ and $P^{\prime}$.

\subsection{2 $\mathrm{S} 19_{\chi} \rightarrow \mathrm{S} 7 / \mathrm{S} 13$}

The Lie algebra is Bianchi $\mathrm{VI}_{\chi}$ for $\chi>0$, which can contract to Bianchi II. The Lie brackets are $[H, B]=(1-\chi) H$ and $[B, P]=(1+\chi) P$. Change basis to $H^{\prime}=H+P$ and $P^{\prime}=P-H$, so that the Lie algebra in this new basis is

$$
\left[H^{\prime}, B\right]=-P^{\prime}-\chi H^{\prime} \quad \text { and } \quad\left[B, P^{\prime}\right]=H^{\prime}+\chi P^{\prime} .
$$

Define $g_{t}$, for $t \in(0,1]$, by

$$
g_{t} \cdot B=t B, \quad g_{t} \cdot H^{\prime}=H^{\prime} \quad \text { and } \quad g_{t} \cdot P^{\prime}=t P^{\prime} .
$$

The brackets become

$$
\left[H^{\prime}, B\right]_{t}=-P^{\prime}-\chi t H^{\prime} \quad \text { and } \quad\left[B, P^{\prime}\right]=t^{2} H^{\prime}+\chi t P^{\prime},
$$

so that the limit $t \rightarrow 0$ recovers the Lie algebra corresponding to spacetime S7/S13.

\subsection{3 $\mathrm{S} 20_{\chi} \rightarrow \mathrm{S} 7 / \mathrm{S} 13$}

The Lie algebra is Bianchi $\mathrm{VII}_{\chi}$ for $\chi>0$, which can contract to Bianchi II. Under the same one-parameter family of invertible transformations $g_{t}$ as in the previous case:

$$
g_{t} \cdot B=t B, \quad g_{t} \cdot H=H \quad \text { and } \quad g_{t} \cdot P=t P,
$$

the brackets become

$$
[H, B]_{t}=P+\chi t H \quad \text { and } \quad[B, P]=t^{2} H-\chi t P .
$$

Taking the limit $t \rightarrow 0$ and changing $B$ to $-B$, recovers the Lie algebra of spacetime S7/S13. 


\subsubsection{Additional limits}

The continua of spacetimes $\mathrm{S} 19_{\chi}$ and $\mathrm{S} 20_{\chi}$ depend on a parameter $\chi>0$. If we take $\chi \rightarrow 0$, then we obtain either Minkowski spacetime or euclidean space: $\mathrm{S} 1=\mathrm{S} 19_{\chi=0}$ and $\mathrm{S} 4=\mathrm{S} 20_{\chi=0}$. The limits $\chi \rightarrow \infty$ are different. Defining $B^{\prime}=\chi^{-1} B$ and letting $\chi \rightarrow \infty$, we obtain the Lie algebra

$$
[B, H]=-H \quad \text { and } \quad[B, P]=-P,
$$

which corresponds to spacetime S18. The only limit of spacetime S18 is to the aristotelian static spacetime A21.

\section{Some geometrical properties of homogeneous spacetimes}

In this section we start to study some of the geometrical properties of the homogeneous spacetimes in tables 1 and 2. We will concentrate on those geometrical properties which are easy to glean from the infinitesimal description in terms of the Lie pair $(\mathfrak{k}, \mathfrak{h})$ and which help distinguish between the different homogeneous spacetimes. In a follow-up paper [54] we will study in more detail the local geometry of these homogeneous spacetimes.

\subsection{Basic notions}

We start by introducing some notions about Lie pairs which are the algebraic analogues of geometric properties of their associated homogeneous spaces.

\subsubsection{Reductive and symmetric Lie pairs}

We say that a Lie pair $(\mathfrak{k}, \mathfrak{h})$ is reductive if there is a vector space decomposition $\mathfrak{k}=\mathfrak{h} \oplus \mathfrak{m}$, where $[\mathfrak{h}, \mathfrak{h}] \subset \mathfrak{h}$ and $[\mathfrak{h}, \mathfrak{m}] \subset \mathfrak{m}$. A reductive Lie pair is said to be symmetric if $[\mathfrak{m}, \mathfrak{m}] \subset \mathfrak{h}$. At the other extreme, if $[\mathfrak{m}, \mathfrak{m}] \subset \mathfrak{m}$, then $\mathfrak{m}$ is a Lie algebra and then the homogeneous space is a principal homogeneous space (i.e., with trivial stabilisers) of a Lie group $\mathcal{M}$ with Lie algebra $\mathfrak{m}$, which is simply-connected if the homogeneous space is. The intersection between two cases is where $[\mathfrak{m}, \mathfrak{m}]=0$. In that case $\mathcal{M}$ is an abelian group and, if simplyconnected, then a vector space. The homogeneous space is then an affine space modelled on $\mathcal{M}$. We will say then that the homogeneous space is affine. (One should not confuse this with the more general notion of an affine symmetric space from $[55,56]$.)

In table 1 we have chosen a basis for $\mathfrak{k}$ in such a way that, in the reductive examples, $\mathfrak{h}$ is spanned by $\boldsymbol{J}$ and $\boldsymbol{B}$ and $\mathfrak{m}$ is the span of $\boldsymbol{P}$ and $H$. It is clear by inspection that all of the spacetimes in table 1 are reductive with one exception: the carrollian light cone LC. We will see below that LC does not admit any invariant connections for $D \geq 2$, providing a separate proof that it is indeed non-reductive. We will also classify the invariant connections for $D=1$.

\subsubsection{The linear isotropy representation}

The natural geometric objects in a homogeneous space (e.g., metric, connections, curvature, torsion,...) are those which are invariant under the group action. Invariance means, in 
particular, that their value at any point is invariant under the stabiliser subgroup of that point, which acts on the tangent space at that point via the linear isotropy representation, which we now introduce at the Lie algebraic level.

Even if $(\mathfrak{k}, \mathfrak{h})$ is not reductive, we have a representation of $\mathfrak{h}$ on $\mathfrak{k} / \mathfrak{h}$, called the linear isotropy representation and denoted $\lambda: \mathfrak{h} \rightarrow \mathfrak{g l}(\mathfrak{k} / \mathfrak{h})$ and sending $X \in \mathfrak{h}$ to $\lambda_{X}$. For every $Y \in \mathfrak{k}$, let $\bar{Y}$ denote its image in $\mathfrak{k} / \mathfrak{h}$. Then $X \in \mathfrak{h}$ acts on $\bar{Y}$ as

$$
\lambda_{X} \bar{Y}:=\overline{[X, Y]}
$$

which is well defined because $\mathfrak{h}$ is a Lie subalgebra. In the reductive case, $\mathfrak{m}$ is isomorphic to $\mathfrak{k} / \mathfrak{h}$ as a representation of $\mathfrak{h}$. In the non-reductive case, we may choose a vector space complement $\mathfrak{m}$ and then define on it a representation of $\mathfrak{h}$ by transporting the linear isotropy representation on $\mathfrak{k} / \mathfrak{h}$ via the vector space isomorphism $\mathfrak{m} \cong \mathfrak{k} / \mathfrak{h}$. In practice all this means is that when calculating the action of $X \in \mathfrak{h}$ on $Y \in \mathfrak{m}$, we can compute the Lie bracket $[X, Y]$ in $\mathfrak{k}$ and set to zero anything on the right-hand side that lies in $\mathfrak{h}$.

If the Lie pair $(\mathfrak{k}, \mathfrak{h})$ has a geometric realisation, the vector space $\mathfrak{k} / \mathfrak{h}$ is an algebraic model for the tangent space at the origin of any homogeneous space $M:=\mathcal{K} / \mathcal{H}$ with $\mathcal{K}$ a Lie group with Lie algebra $\mathfrak{k}$ and $\mathcal{H}$ a Lie subgroup with Lie algebra $\mathfrak{h}$. If $\mathcal{H}$ is connected - which we can assume with no loss of generality by passing to the universal cover of the homogeneous space, if necessary - there is then a bijective correspondence between $\mathfrak{h}$-invariant tensors on $\mathfrak{k} / \mathfrak{h}$ and $\mathcal{G}$-invariant tensor fields on $M$.

\subsubsection{Invariant structures}

For the purposes of this paper, we are particularly interested in invariant tensors of low rank; that is, $\mathfrak{h}$-invariant tensors in $\mathfrak{k} / \mathfrak{h},(\mathfrak{k} / \mathfrak{h})^{*}, S^{2}(\mathfrak{k} / \mathfrak{h})$ and $S^{2}(\mathfrak{k} / \mathfrak{h})^{*}$. A non-degenerate invariant tensor in $S^{2}(\mathfrak{k} / \mathfrak{h})^{*}$ gives rise to a $\mathcal{G}$-invariant metric on $M$, whereas $\mathfrak{h}$-invariant tensors in $\mathfrak{k} / \mathfrak{h}$ and $(\mathfrak{k} / \mathfrak{h})^{*}$ give rise to an invariant vector field and one-form on $M$, respectively. We shall say that a homogeneous kinematical spacetime $M$ is lorentzian or riemannian if it admits a $\mathcal{G}$-invariant metric of lorentzian or riemannian signature. This is the case if the associated Lie pair admits an $\mathfrak{h}$-invariant nondegenerate tensor in $S^{2}(\mathfrak{k} / \mathfrak{h})^{*}$ of the right signature. In cases where rotations are present in $\mathfrak{h}$ (i.e., $D \geq 2$ ), the only possible invariant in $\mathfrak{k} / \mathfrak{h}$ must be proportional to $\bar{H}$ and any invariants in $S^{2}(\mathfrak{k} / \mathfrak{h})$ must lie in the subspace spanned by $\bar{H}^{2}$ and $\bar{P}^{2}:=\delta^{a b} \bar{P}_{a} \bar{P}_{b}$.

Let us introduce a basis $\left(\pi^{a}, \eta\right)$ for $(\mathfrak{k} / \mathfrak{h})^{*}$ canonically dual to the basis $\left(\bar{P}_{a}, \bar{H}\right)$ for $\mathfrak{k} / \mathfrak{h}$; that is,

$$
\pi^{a}\left(\bar{P}_{b}\right)=\delta_{b}^{a} \quad \pi^{a}(\bar{H})=0 \quad \eta\left(\bar{P}_{a}\right)=0 \quad \eta(\bar{H})=1 .
$$

Then similarly, for $D \geq 2$, any invariants in $(\mathfrak{k} / \mathfrak{h})^{*}$ are proportional to $\eta$, whereas in $S^{2}(\mathfrak{k} / \mathfrak{h})^{*}$ any invariant lies in the span of $\eta^{2}$ and $\pi^{2}$, where $\pi^{2}=\delta_{a b} \pi^{a} \pi^{b}$.

If $(\mathfrak{k}, \mathfrak{h})$ is such that the one-form $\eta \in(\mathfrak{k} / \mathfrak{h})^{*}$ and the co-metric $\bar{P}^{2} \in S^{2}(\mathfrak{k} / \mathfrak{h})$ are $\mathfrak{h}$ invariant, we say that $M$ admits an invariant galilean structure. The one-form $\eta$ is called the absolute clock reflecting that galilean spacetimes are absolute in time. This means, two points of the homogeneous space that are at same time stay that way, irrespective of any galilean kinematical transformation. If $\bar{H} \in \mathfrak{k} / \mathfrak{h}$ and $\pi^{2} \in S^{2}(\mathfrak{k} / \mathfrak{h})^{*}$ are $\mathfrak{h}$-invariant we 
say that $M$ admits an invariant carrollian structure. Analogous to the galilean case, the fundamental vector field $\bar{H}$ reflects the absolute space character of carrollian spacetimes. Notice that aristotelian spacetimes in table 2 admit simultaneously a galilean and carrollian structure, since $\bar{H}, \eta, \bar{P}^{2}, \pi^{2}$ are all rotationally invariant. It follows that they also admit many invariant lorentzian and riemannian structures.

It is easy to determine the existence of these invariants from the data in table 1 . After writing down down the possible rotationally invariant tensors, we only need to check invariance under $\boldsymbol{B}$. The action of $\boldsymbol{B}$ is induced by duality from its action via the linear isotropy representation on $\mathfrak{g} / \mathfrak{h}$ :

$$
\lambda_{B_{a}} \bar{H}=\overline{\left[B_{a}, H\right]} \quad \text { and } \quad \lambda_{B_{a}} \bar{P}_{b}=\overline{\left[B_{a}, P_{b}\right]}
$$

with the brackets being those of $\mathfrak{k}$. In practice, we can determine this from the tables, by computing the brackets in $\mathfrak{k}$ and simply dropping any $\boldsymbol{B}$ or $\boldsymbol{J}$ from the right-hand side.

The only possible invariants in $\mathfrak{k} / \mathfrak{h}$ are proportional to $\bar{H}$, which is invariant provided that $[\boldsymbol{B}, H] \in \mathfrak{h}$. Dually, the only possible invariants in $(\mathfrak{k} / \mathfrak{h})^{*}$ are proportional to $\eta$, which is invariant provided that there is no $X \in \mathfrak{k}$ such that $H$ appears in $[\boldsymbol{B}, X]$.

\subsubsection{Parity and time reversal}

We define a parity transformation on a kinematical Lie pair $(\mathfrak{k}, \mathfrak{h})$ to be an automorphism of $\mathfrak{k}$ which changes the sign of $\boldsymbol{B}$ and $\boldsymbol{P}$ and leaves $H$ and $\boldsymbol{J}$ inert. Similarly, we define a time reversal transformation to be an automorphism of $\mathfrak{k}$ which changes the sign of $\boldsymbol{B}$ and $H$, but leaves $\boldsymbol{P}$ and $\boldsymbol{J}$ inert. For aristotelian Lie pairs $(\mathfrak{a}, \mathfrak{r})$, only $\boldsymbol{P}$ changes sign under a parity transformation and only $H$ changes sign under a time reversal transformation. The combination of a parity and time-reversal transformations is then an automorphism of $\mathfrak{k}$ (or $\mathfrak{a}$ ) which changes simultaneously the signs of $\boldsymbol{P}$ and $H$ and leaves other generators inert.

It follows from equivariance under rotations, that for $D \neq 1,3$, every kinematical Lie algebra possesses a parity transformation. It is only in $D=1$ and $D=3$ where we can have Lie brackets which violate parity: in $D=3$ because we have a vector product which is only invariant under the orientation preserving orthogonal transformations, and in $D=1$ because there are no rotations. We saw that there are no effective Lie pairs for $D=3$ whose kinematical Lie algebra involves the vector product, so that it is only in $D=1$ where we can expect to have effective Lie pairs without parity (or time reversal, since in $D=1$ what is time and space is a matter of convention) symmetry.

Any spacetime whose canonical connection (see section 6.2 below) has torsion cannot be invariant under $P T$, hence it cannot be invariant under both $P$ and $T$. Since, as explained above, parity is guaranteed for $D \neq 1$, it is time reversal invariance which fails for torsional geometries.

It is simply a matter of inspecting the brackets in tables 1 and 2 to determine whether the corresponding spacetimes possess parity and/or time reversal invariance. The results are summarised in table 16. 


\subsection{Invariant connections, curvature and torsion}

As a final geometric property, we discuss the existence of invariant affine connections and their curvature and torsion. Let $(\mathfrak{k}, \mathfrak{h})$ be an effective Lie pair associated to a homogeneous space. By an invariant (affine) connection on $(\mathfrak{k}, \mathfrak{h})$ we shall mean a linear map $\Lambda: \mathfrak{k} \rightarrow$ $\mathfrak{g l}(\mathfrak{k} / \mathfrak{h})$, denoted $X \mapsto \Lambda_{X}$, satisfying the following two properties:

1. $\left.\Lambda\right|_{\mathfrak{h}}=\lambda$, the linear isotropy representation, and

2. $\Lambda$ is $\mathfrak{h}$-equivariant.

Notice that $\Lambda$ is not generally a Lie algebra homomorphism; although its restriction to $\mathfrak{h}$ is. In fact, as we will see, the obstruction of $\Lambda$ being a Lie algebra homomorphism is the curvature of the connection.

The equivariance condition for $\Lambda$ says that for all $X, Y \in \mathfrak{k}$ and $Z \in \mathfrak{h}$,

$$
0=\lambda_{Z} \Lambda_{X} \bar{Y}-\Lambda_{X} \lambda_{Z} \bar{Y}-\Lambda_{[Z, X]} \bar{Y},
$$

which is the $\mathfrak{h}$-invariance of $\Lambda$ as an element in the space of linear maps $\operatorname{Hom}(\mathfrak{k}, \mathfrak{g l}(\mathfrak{k} / \mathfrak{h}))$. Notice that if either $X$ or $Y$ are in $\mathfrak{h}$, then this is automatically satisfied: it is clear if $Y \in \mathfrak{h}$, since then $\bar{Y}=0$, but then also if $X \in \mathfrak{h}, \Lambda_{X}=\lambda_{X}$ and the invariance condition reads

$$
0=\lambda_{Z} \lambda_{X} \bar{Y}-\lambda_{X} \lambda_{Z} \bar{Y}-\lambda_{[Z, X]} \bar{Y},
$$

which holds because $\lambda$ is a representation. So the only nontrivial condition comes from $X, Y \notin \mathfrak{h}$.

Now choose $\mathfrak{m}$ such that $\mathfrak{k}=\mathfrak{h} \oplus \mathfrak{m}$ as vector spaces. In the reductive case, we can choose $\mathfrak{m}$ such that $[\mathfrak{h}, \mathfrak{m}] \subset \mathfrak{m}$ and hence $\mathfrak{m}$ is an $\mathfrak{h}$-module. But even in the non-reductive case, $\mathfrak{m} \cong \mathfrak{k} / \mathfrak{h}$ as a vector space and there is a unique way to give $\mathfrak{m}$ the structure of an $\mathfrak{h}$-module so that this is also an isomorphism of $\mathfrak{h}$-modules. Let us assume we have done that.

Now let $\Lambda$ be an invariant affine connection. Since the restriction of $\Lambda$ to $\mathfrak{h}$ is fixed, $\Lambda$ is determined by its component mapping $\mathfrak{m} \rightarrow \mathfrak{g l}(\mathfrak{k} / \mathfrak{h})$ and, as argued above, it is only this component that is involved in the invariance condition. In the reductive case, this component is an $\mathfrak{h}$-equivariant linear map $\mathfrak{m} \rightarrow \mathfrak{g l}(\mathfrak{m})$ or, equivalently, an $\mathfrak{h}$-equivariant bilinear map $\mathfrak{m} \times \mathfrak{m} \rightarrow \mathfrak{m}$, called the Nomizu map. In a reductive homogeneous space, we may always take the Nomizu map to be zero and in this way arrive at a canonical invariant connection (termed "of the second kind" in [55]). All invariant connections are then classified by their Nomizu maps.

In the non-reductive case, it can very well be the case that there are no invariant connections. Turning this around, if a given homogeneous space does not admit any invariant connections, it cannot be reductive.

Given an invariant affine connection, its torsion is given for all $X, Y \in \mathfrak{k}$,

$$
\Theta(X, Y)=\Lambda_{X} \bar{Y}-\Lambda_{Y} \bar{X}-\overline{[X, Y]} .
$$

One checks that this only depends on the images $\bar{X}, \bar{Y}$ of $X, Y$ in $\mathfrak{k} / \mathfrak{h}$, so it defines an $\mathfrak{h}$-equivariant skewsymmetric bilinear map $\Theta: \mathfrak{k} / \mathfrak{h} \times \mathfrak{k} / \mathfrak{h} \rightarrow \mathfrak{k} / \mathfrak{h}$. 
The curvature $\Omega$ is given, for all $X, Y \in \mathfrak{k}$ and $\bar{Z} \in \mathfrak{k} / \mathfrak{h}$, by

$$
\Omega(X, Y) \bar{Z}=\left[\Lambda_{X}, \Lambda_{Y}\right] \bar{Z}-\Lambda_{[X, Y]} \bar{Z},
$$

from where we see that it measures the failure of $\Lambda: \mathfrak{k} \rightarrow \mathfrak{g l}(\mathfrak{k} / \mathfrak{h})$ to be a Lie algebra homomorphism. The $\mathfrak{h}$-equivariance of $\Lambda$ guarantees that this expression only depends on $\bar{X}, \bar{Y}$ and hence it defines an $\mathfrak{h}$-equivariant skewsymmetric bilinear map $\Omega: \mathfrak{k} / \mathfrak{h} \times \mathfrak{k} / \mathfrak{h} \rightarrow$ $\mathfrak{g l}(\mathfrak{k} / \mathfrak{h})$. When the curvature vanishes we say that the canonical connection is flat.

In the reductive case, the torsion and curvature of an invariant affine connection are given in terms of its Nomizu map $\alpha: \mathfrak{m} \times \mathfrak{m} \rightarrow \mathfrak{m}$ by the following expressions for all $X, Y, Z \in \mathfrak{m}$,

$$
\begin{aligned}
\Theta(X, Y) & =\alpha(X, Y)-\alpha(Y, X)-[X, Y]_{\mathfrak{m}}, \\
\Omega(X, Y) Z & =\alpha(X, \alpha(Y, Z))-\alpha(Y, \alpha(X, Z))-\alpha\left([X, Y]_{\mathfrak{m}}, Z\right)-\left[[X, Y]_{\mathfrak{h}}, Z\right],
\end{aligned}
$$

where $[X, Y]=[X, Y]_{\mathfrak{h}}+[X, Y]_{\mathfrak{m}}$ is the decomposition of $[X, Y] \in \mathfrak{k}=\mathfrak{h} \oplus \mathfrak{m}$. These expressions simplify for the canonical connection with zero Nomizu map:

$$
\Theta(X, Y)=-[X, Y]_{\mathfrak{m}} \quad \text { and } \quad \Omega(X, Y) Z=-\left[[X, Y]_{\mathfrak{h}}, Z\right] .
$$

If the space is symmetric, so that $[X, Y] \in \mathfrak{h}$ for $X, Y \in \mathfrak{m}$, then we see that the canonical connection is torsion-free. If, on the contrary, the canonical connection is flat, then $[X, Y] \in$ $\mathfrak{m}$ for all $X, Y \in \mathfrak{m}$ and hence $\mathfrak{m}$ is a Lie subalgebra of $\mathfrak{k}$. In this case we can identify the homogeneous space (assumed simply-connected) with the group manifold of the simplyconnected Lie group with Lie algebra $\mathfrak{m}$. If the canonical connection is both torsion-free and flat, then the Lie algebra $\mathfrak{m}$ is abelian and hence the homogeneous space (if simplyconnected) is the group manifold of a simply-connected abelian Lie group. A simplyconnected abelian Lie group is a vector space and hence the homogeneous space in that case is an affine space.

The holonomy of the canonical connection on a reductive homogeneous space $M=\mathcal{G} / \mathcal{H}$ can be calculated in general. Indeed, by a result of Nomizu's [55, §12], the Lie algebra of the holonomy group is isomorphic to the ideal $[\mathfrak{m}, \mathfrak{m}]_{\mathfrak{h}}$ of $\mathfrak{h}$ acting on $\mathfrak{m}$ via (the restriction of) the linear isotropy representation. As a corollary, the covariant derivative of a $\mathcal{G}$-invariant tensor field $T$, say, on $M$ with respect to the canonical connection vanishes: $\nabla T=0$. If $M$ is simply-connected, then parallel-transporting $T$ along any closed loop leaves it invariant. In particular, the curvature and torsion tensor fields (of any invariant connection, but in particular of the canonical connection), being themselves invariant, are parallel, and so are the tensor fields corresponding to any invariant lorentzian, riemannian, galilean, carrollian or aristotelian structure that $M$ might possess. In particular the connection is compatible with the (co-)metrics. It is thus clear that the galilean $(\mathrm{G})$ and carrollian $(\mathrm{C})$ spacetimes reproduce the standard flat Newton-Cartan and Carroll structures, respectively (cf. [39]).

All spacetimes, except for LC are reductive. It is then easy to inspect tables 1 and 2 and determine the torsion and curvature of the canonical invariant connection with zero Nomizu map. The results are summarised in section 6.3 and in table 16. The existence of invariant connections for the non-reductive spacetime LC has to be studied separately and 
we do so below. We will see that for $D \geq 2$ the spacetime admits no invariant connections, whereas for $D=1$ it admits a three-parameter family of invariant connections and a unique torsion-free, flat connection. The follow-up paper [54] presents the classification of invariant connections and the calculation of their torsion and curvature for the reductive kinematical and aristotelian spacetimes.

\subsubsection{Invariant connections for spacetime LC}

We will show that this homogeneous spacetime does not admit any invariant connections for $D \geq 2$. Since $\left[H, B_{a}\right]=B_{a},\left[H, P_{a}\right]=-P_{a}$ and $\left[B_{a}, P_{b}\right]=\delta_{a b} H+J_{a b}$, we have that $\lambda_{B_{a}} \bar{H}=0$ and $\lambda_{B_{a}} \bar{P}_{b}=\delta_{a b} \bar{H}$.

$(\boldsymbol{D} \geq \mathbf{3})$. The most general rotationally equivariant map $\Lambda$ is given by

$$
\Lambda_{H} \bar{H}=\alpha \bar{H} \quad \Lambda_{H} \bar{P}_{a}=\beta \bar{P}_{a} \quad \Lambda_{P_{a}} \bar{H}=\gamma \bar{P}_{a} \quad \text { and } \quad \Lambda_{P_{a}} \bar{P}_{b}=\mu \delta_{a b} \bar{H}+\nu \epsilon_{a b c} \bar{P}_{c}
$$

with the tacit understanding that the term proportional to $\nu$ is only present if $D=3$. Invariance demands, in particular, that

$$
0=\lambda_{B_{a}} \Lambda_{P_{b}} \bar{P}_{c}-\Lambda_{\left[B_{a}, P_{b}\right]} \bar{P}_{c}-\Lambda_{P_{b}} \lambda_{B_{a}} \bar{P}_{c}
$$

which becomes

$$
\begin{aligned}
0 & =\nu \epsilon_{a b c} \bar{H}-\delta_{a b} \Lambda_{H} \bar{P}_{c}-\Lambda_{J_{a b}} \bar{P}_{c}-\delta_{a c} \Lambda_{P_{b}} \bar{H} \\
& =\nu \epsilon_{a b c} \bar{H}-\beta \delta_{a b} \bar{P}_{c}-\left(\delta_{b c} \bar{P}_{a}-\delta_{a c} \bar{P}_{b}\right)-\gamma \delta_{a c} \bar{P}_{b} \\
& =\nu \epsilon_{a b c} \bar{H}-\beta \delta_{a b} \bar{P}_{c}-\delta_{b c} \bar{P}_{a}+(1-\gamma) \delta_{a c} \bar{P}_{b} .
\end{aligned}
$$

Taking any $b=c \neq a$, we arrive at a contradiction. Therefore there are no invariant connections for $D \geq 3$.

$(\boldsymbol{D}=\mathbf{2})$. Now the most general rotationally equivariant $\Lambda$ is given by

$$
\begin{aligned}
\Lambda_{H} \bar{H} & =\alpha \bar{H} & \Lambda_{P_{a}} \bar{H} & =\gamma \bar{P}_{a}+\gamma^{\prime} \epsilon_{a b} \bar{P}_{b} \\
\Lambda_{H} \bar{P}_{a} & =\beta \bar{P}_{a}+\beta^{\prime} \epsilon_{a b} \bar{P}_{b} & \Lambda_{P_{a}} \bar{P}_{b} & =\mu \delta_{a b} \bar{H}+\mu^{\prime} \epsilon_{a b} \bar{H} .
\end{aligned}
$$

But as before, equivariance requires, in particular, that

$$
0=\lambda_{B_{a}} \Lambda_{P_{b}} \bar{P}_{c}-\Lambda_{\left[B_{a}, P_{b}\right]} \bar{P}_{c}-\Lambda_{P_{b}} \lambda_{B_{a}} \bar{P}_{c}
$$

which again for any $b=c \neq a$ results in a contradiction. Therefore there are no invariant connections for $D=2$ either.

$(\boldsymbol{D}=\mathbf{1})$. Now there are no rotations, and $\Lambda$ is a general linear map:

$$
\begin{array}{ll}
\Lambda_{H} \bar{H}=\alpha \bar{H}+\alpha^{\prime} \bar{P} & \Lambda_{P} \bar{H}=\gamma \bar{H}+\gamma^{\prime} \bar{P} \\
\Lambda_{H} \bar{P}=\beta \bar{H}+\beta^{\prime} \bar{P} & \Lambda_{P} \bar{P}=\delta \bar{H}+\delta^{\prime} \bar{P} .
\end{array}
$$

Invariance under $B$ says that for all $X, Y \in\{H, P\}$,

$$
\lambda_{B} \Lambda_{X} \bar{Y}-\Lambda_{[B, X]} \bar{Y}-\Lambda_{X} \lambda_{B} \bar{Y}=0 .
$$


Taking $(X, Y)=(H, H),(H, P),(P, H),(P, P)$ in turn we arrive at the following conditions:

$$
\alpha^{\prime}=0 \quad \beta^{\prime}=\alpha-1 \quad \gamma^{\prime}=\alpha \quad \delta^{\prime}=\beta+\gamma \quad \text { and } \quad \beta^{\prime}+\gamma^{\prime}=0 .
$$

This results in the following three-parameter family of invariant connections

$$
\begin{aligned}
\Lambda_{H} \bar{H} & =\frac{1}{2} \bar{H} & \Lambda_{P} \bar{H} & =\gamma \bar{H}+\frac{1}{2} \bar{P} \\
\Lambda_{H} \bar{P} & =\beta \bar{H}-\frac{1}{2} \bar{P} & \Lambda_{P} \bar{P} & =\delta \bar{H}+(\beta+\gamma) \bar{P} .
\end{aligned}
$$

Calculating the torsion and curvature, we find

$$
\begin{aligned}
\Theta(H, P) & =(\beta-\gamma) \bar{H} \\
\Omega(H, P) \bar{H} & =\left(\gamma+\frac{1}{2} \beta\right) \bar{H} \quad \text { and } \quad \Omega(H, P) \bar{P}=\left(2 \delta+\beta^{2}\right) \bar{H}+\left(\frac{1}{2} \beta+\gamma\right) \bar{P} .
\end{aligned}
$$

We see that there is a unique flat and torsion-free connection, given by

$$
\begin{aligned}
\Lambda_{H} \bar{H} & =\frac{1}{2} \bar{H} & \Lambda_{P} \bar{H} & =\frac{1}{2} \bar{P} \\
\Lambda_{H} \bar{P} & =-\frac{1}{2} \bar{P} & \Lambda_{P} \bar{P} & =0 .
\end{aligned}
$$

\subsection{Summary of properties of homogeneous spacetimes}

We now summarise the properties of the simply-connected homogeneous spacetimes in tables 1 and 2 .

There is precisely one non-reductive spacetime: LC (S16), which we identified with the future light cone in Minkowski spacetime one dimension higher. It is carrollian, but for $D>1$ does not admit any invariant connections. We determined above the invariant connections when $D=1$.

The remaining spacetimes in tables 1 and 2 are reductive and we proceed to list them according to the type of reductive structure they possess.

\subsubsection{Flat symmetric spacetimes}

These are symmetric spacetimes where the canonical connection is flat $(\Omega=0)$. This means that the homogeneous space is a principal homogeneous space for the translations. If simply-connected, then it is an affine space modelled on the vector space of translations.

(S1) Minkowski spacetime

(S4) euclidean space

(S7) galilean spacetime

(S13) carrollian spacetime

(A21) static aristotelian spacetime

and the exotic two-dimensional spacetimes: (S17), (S18), (S19) $\chi>0$ and (S20) $\chi>0$. 


\subsubsection{Non-flat symmetric spacetimes}

(S2) de Sitter spacetime, with curvature

$$
\Omega\left(H, P_{a}\right)=\lambda_{B_{a}} \quad \text { and } \quad \Omega\left(P_{a}, P_{b}\right)=\lambda_{J_{a b}} .
$$

The notation is such that we interpret $\Omega$ as a two-form with values in endomorphisms of the tangent space, which for a homogeneous space localises to a linear map $\Omega: \Lambda^{2} \mathfrak{m} \rightarrow \mathfrak{g l}(\mathfrak{m})$ and we write it in terms of the image in $\mathfrak{g l}(\mathfrak{m})$ of the linear isotropy representation $\lambda: \mathfrak{h} \rightarrow \mathfrak{g l}(\mathfrak{m})$. For example,

$$
\Omega\left(H, P_{a}\right) H=\lambda_{B_{a}} H=\left[B_{a}, H\right]=P_{a} \quad \text { and } \quad \Omega\left(H, P_{a}\right) P_{b}=\lambda_{B_{a}} P_{b}=\delta_{a b} H,
$$

et cetera. Notice that the curvature 2 -form of the canonical connection of de Sitter spacetime does not see the radius of curvature. This is because the canonical connection (and hence its curvature) is an invariant of the reductive homogeneous space, whereas the radius of curvature is an additional structure: namely, an invariant lorentzian metric. The same happens with anti de Sitter spacetime, the round sphere and hyperbolic space.

(S3) anti de Sitter spacetime, with curvature

$$
\Omega\left(H, P_{a}\right)=-\lambda_{B_{a}} \quad \text { and } \quad \Omega\left(P_{a}, P_{b}\right)=-\lambda_{J_{a b}},
$$

which is formally like for de Sitter spacetime except for an overall sign.

(S5) round sphere, with curvature formally identical to that in equation (6.21), except that of course, the linear isotropy representation $\lambda_{B_{a}}$ differs.

(S6) hyperbolic space, with curvature formally identical to that in equation (6.23), except that again the linear isotropy representation $\lambda_{B_{a}}$ differs.

(S8) galilean de Sitter spacetime, with curvature

$$
\Omega\left(H, P_{a}\right)=\lambda_{B_{a}} .
$$

(S10) galilean anti de Sitter spacetime, with curvature

$$
\Omega\left(H, P_{a}\right)=-\lambda_{B_{a}},
$$

which is again a sign off the one for galilean de Sitter spacetime.

(S14) carrollian de Sitter spacetime, whose curvature is formally identical to that in equation (6.21), with the different action of $\lambda_{B_{a}}$.

(S15) carrollian anti de Sitter spacetime, whose curvature is formally identical to that in equation (6.23), with the different action of $\lambda_{B_{a}}$.

(A23) aristotelian: $\mathbb{R} \times \mathbb{S}^{D}$ for $\varepsilon=-1$ and $\mathbb{R} \times \mathbb{H}^{D}$ for $\varepsilon=1$, with curvature given by

$$
\Omega\left(P_{a}, P_{b}\right)=-\varepsilon \lambda_{J_{a b}} .
$$




\subsubsection{Reductive torsional spacetimes}

The canonical connection of these reductive spacetimes has torsion and hence they are not symmetric spaces. If the connection is flat, then the spacetime is actually a principally homogeneous space for a Lie group whose Lie algebra is isomorphic to $\mathfrak{m}$, which is a Lie algebra in the flat case.

(S9) This is $\mathrm{dSG}_{\gamma}$ for $\gamma \in(-1,1]$. The torsion and curvature of the canonical connection are given by

$$
\Theta\left(H, P_{a}\right)=-(1+\gamma) P_{a} \quad \text { and } \quad \Omega\left(H, P_{a}\right)=-\gamma \lambda_{B_{a}} .
$$

We see that it is torsion-free if and only if $\gamma=-1$, which corresponds to the symmetric space (S8). It is flat if and only if $\gamma=0$. It is then a principally homogeneous space for the simply-connected solvable Lie group with Lie algebra $\left[H, P_{a}\right]=P_{a}$.

(S11) This is $\mathrm{AdSG}_{\chi}$ for $\chi>0$. The torsion and curvature of the canonical connection are given by

$$
\Theta\left(H, P_{a}\right)=-2 \chi P_{a} \quad \text { and } \quad \Omega\left(H, P_{a}\right)=-\left(1+\chi^{2}\right) \lambda_{B_{a}}
$$

so that it is never flat, but it is torsion-free if and only if $\chi=0$, corresponding to the symmetric space (S10).

(S12) This is a two-parameter family of three-dimensional galilean spacetimes $\mathrm{S} 12_{\gamma, \chi}$ with $\gamma \in[-1,1)$ and $\chi>0$. The torsion and curvature are given by

$$
\Theta\left(H, P_{a}\right)=-(1+\gamma) P_{a}+\chi \epsilon_{a b} P_{b} \quad \text { and } \quad \Omega\left(H, P_{a}\right)=-\gamma \lambda_{B_{a}}+\chi \epsilon_{a b} \lambda_{B_{b}}, \text { (6.29) }
$$

which is torsion-free if and only if $\gamma=-1$ and $\chi=0$, which corresponds to galilean de Sitter spacetime (S8). The connection is flat if and only if $\gamma=\chi=0$, which corresponds to $\mathrm{S} 9_{\gamma=0}$.

(A22) This is an aristotelian non-symmetric space with torsion

$$
\Theta\left(H, P_{a}\right)=-P_{a}
$$

and zero curvature. It is a principally homogeneous space for the simply-connected solvable Lie group with Lie algebra $\left[H, P_{a}\right]=P_{a}$.

(A24) This is a three-dimensional aristotelian spacetime with torsion

$$
\Theta\left(P_{a}, P_{b}\right)=-\epsilon_{a b} H
$$

and zero curvature. It is a principally homogeneous space for the simply-connected Heisenberg Lie group with Lie algebra $\left[P_{a}, P_{b}\right]=\epsilon_{a b} H$. 


\subsubsection{Summary}

In table 16 we summarise the basic properties of the homogeneous kinematical spacetimes in table 1 and aristotelian spacetimes in table 2. The first column is simply our label in this paper, the second column specifies the value of $D$, where the dimension of the spacetime is $D+1$. For the columns labeled "R", "S" and "A" we indicate with a $\checkmark$ when a spacetime is reductive, symmetric and/or affine, respectively. The columns labelled "L", "E", "G" and " $\mathrm{C}$ " indicate the kind of invariant structures the spacetime possesses: lorentzian, riemannian ("euclidean"), galilean and carrollian, respectively. Again a $\checkmark$ indicates that the spacetime possesses that structure. The columns "P", "T" and "PT" indicate whether the spacetime is invariant under parity, time reversal or their combination, respectively, with $\checkmark$ signalling when they are. The columns " $\Omega$ " and " $\Theta$ " tell us, respectively, about the curvature and torsion of the canonical invariant connection for the reductive spacetimes (that is, all but S16). A $\neq 0$ indicates the presence of curvature and/or torsion. Otherwise the connection is flat and/or torsion-free, respectively. The final column contains any relevant comments, including, when known, the name of the spacetime.

The table is divided into six sections. The first four correspond to lorentzian, euclidean, galilean and carrollian spacetimes. The fifth section contains two-dimensional spacetimes with no invariant structure of these kinds. The sixth and last section contains the aristotelian spacetimes.

In particular, we see how all the spacetimes in figure 2 are indeed symmetric: with

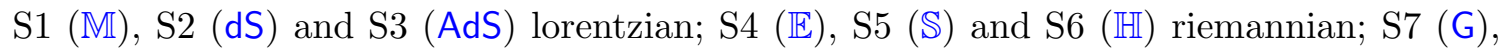
S8 (dSG) and S10 (AdSG) galilean; and S13 (C), S14 (dSC) and S15 (AdSC) carrollian. It is clear from a dimension count that there can be no other lorentzian or riemannian kinematical spacetimes than the ones in figure 2. The dimension of the kinematical group associated to a $(D+1)$-dimensional homogeneous spacetime is given by $(D+1)(D+2) / 2$, which is also the maximal dimension of the isometry group of a $(D+1)$-dimensional pseudoriemannian manifold, so the homogeneous lorentzian and riemannian homogeneous spaces are necessarily maximally symmetric. The perhaps remarkable fact is that for $D \geq 2$, every homogeneous (kinematical) spacetime which is not lorentzian or riemannian is either galilean, carrollian or aristotelian. The new spacetimes in figures 3 and 4, not already present in figure 2, are therefore necessarily galilean, carrollian or aristotelian. We see that the class of galilean spacetimes is particularly rich: admitting two one-dimensional continua of such spacetimes: one, denoted $\mathrm{AdSG}_{\chi}\left(\mathrm{S}_{1} 1_{\chi}\right)$, which extends the galilean anti de Sitter spacetime AdSG (S10) and a second, denoted $\mathrm{dSG}_{\gamma}\left(\mathrm{S} 9_{\gamma}\right)$, which extends the galilean de Sitter spacetime dSG (S8). The carrollian spacetimes are all realisable as null hypersurfaces in either Minkowski or (anti) de Sitter spacetimes one dimension higher. In particular, the carrollian spacetime LC (S16) can be realised as the future light cone in Minkowski space one dimension higher. There are also several aristotelian spacetimes. The situation in $D=2$ is even richer, with a two-dimensional continuum interpolating between the one-dimensional continua present in all $D \geq 1$. Finally, in $D=1$ there are exotic (i.e., without any discernible invariant structures) homogeneous spacetimes, including two one-dimensional continua. 


\begin{tabular}{|c|c|c|c|c|c|c|c|c|c|c|c|c|c|c|}
\hline Label & $D$ & $R$ & $S$ & $A$ & $L$ & $E$ & $G$ & $C$ & $P$ & $T$ & $P T$ & $\Omega$ & $\Theta$ & Comments \\
\hline S1 & $\geq 1$ & $\checkmark$ & $\checkmark$ & $\checkmark$ & $\checkmark$ & & & & $\checkmark$ & $\checkmark$ & $\checkmark$ & & & Minkowski \\
\hline $\mathrm{S} 2$ & $\geq 1$ & $\checkmark$ & $\checkmark$ & & $\checkmark$ & & & & $\checkmark$ & $\checkmark$ & $\checkmark$ & $\neq 0$ & & de Sitter \\
\hline S3 & $\geq 1$ & $\checkmark$ & $\checkmark$ & & $\checkmark$ & & & & $\checkmark$ & $\checkmark$ & $\checkmark$ & $\neq 0$ & & anti de Sitter \\
\hline $\mathrm{S} 4$ & $\geq 1$ & $\checkmark$ & $\checkmark$ & $\checkmark$ & & $\checkmark$ & & & $\checkmark$ & $\checkmark$ & $\checkmark$ & & & euclidean \\
\hline S5 & $\geq 1$ & $\checkmark$ & $\checkmark$ & & & $\checkmark$ & & & $\checkmark$ & $\checkmark$ & $\checkmark$ & $\neq 0$ & & sphere \\
\hline S6 & $\geq 1$ & $\checkmark$ & $\checkmark$ & & & $\checkmark$ & & & $\checkmark$ & $\checkmark$ & $\checkmark$ & $\neq 0$ & & hyperbolic \\
\hline S7 & $\geq 1$ & $\checkmark$ & $\checkmark$ & $\checkmark$ & & & $\checkmark$ & & $\checkmark$ & $\checkmark$ & $\checkmark$ & & & galilean \\
\hline S8 & $\geq 1$ & $\checkmark$ & $\checkmark$ & & & & $\checkmark$ & & $\checkmark$ & $\checkmark$ & $\checkmark$ & $\neq 0$ & & galilean $\mathrm{dS}=\mathrm{dSG}_{-1}$ \\
\hline $\mathrm{S} 9_{\gamma \neq 0}$ & $\geq 1$ & $\checkmark$ & & & & & $\checkmark$ & & $\checkmark$ & & & $\neq 0$ & $\neq 0$ & $\mathrm{dSG}_{\gamma}, 0 \neq \gamma \in(-1,1]$ \\
\hline $\mathrm{S} 9_{0}$ & $\geq 1$ & $\checkmark$ & & & & & $\checkmark$ & & $\checkmark$ & & & & $\neq 0$ & $\mathrm{dSG}_{0}$ \\
\hline S10 & $\geq 1$ & $\checkmark$ & $\checkmark$ & & & & $\checkmark$ & & $\checkmark$ & $\checkmark$ & $\checkmark$ & $\neq 0$ & & galilean $\mathrm{AdS}=\mathrm{AdSG}_{0}$ \\
\hline $\mathrm{S} 11_{\chi}$ & $\geq 1$ & $\checkmark$ & & & & & $\checkmark$ & & $\checkmark$ & & & $\neq 0$ & $\neq 0$ & $\mathrm{AdSG}_{\chi}, \chi>0$ \\
\hline $\mathrm{S} 12_{\gamma, \chi}$ & 2 & $\checkmark$ & & & & & $\checkmark$ & & $\checkmark$ & & & $\neq 0$ & $\neq 0$ & $\gamma \in[-1,1), \chi>0$ \\
\hline S13 & $\geq 1$ & $\checkmark$ & $\checkmark$ & $\checkmark$ & & & & $\checkmark$ & $\checkmark$ & $\checkmark$ & $\checkmark$ & & & carrollian \\
\hline S14 & $\geq 1$ & $\checkmark$ & $\checkmark$ & & & & & $\checkmark$ & $\checkmark$ & $\checkmark$ & $\checkmark$ & $\neq 0$ & & carrollian dS \\
\hline S15 & $\geq 1$ & $\checkmark$ & $\checkmark$ & & & & & $\checkmark$ & $\checkmark$ & $\checkmark$ & $\checkmark$ & $\neq 0$ & & carrollian AdS \\
\hline S16 & $\geq 1$ & & & & & & & $\checkmark$ & $\checkmark$ & & & & & carrollian light cone \\
\hline S17 & 1 & $\checkmark$ & $\checkmark$ & $\checkmark$ & & & & & & & $\checkmark$ & & & \\
\hline S18 & 1 & $\checkmark$ & $\checkmark$ & $\checkmark$ & & & & & & $\checkmark$ & & & & \\
\hline $\mathrm{S} 19 x$ & 1 & $\checkmark$ & $\checkmark$ & $\checkmark$ & & & & & & $\checkmark$ & & & & $\chi>0$ \\
\hline $\mathrm{S} 20_{\chi}$ & 1 & $\checkmark$ & $\checkmark$ & $\checkmark$ & & & & & & & $\checkmark$ & & & $\chi>0$ \\
\hline A21 & $\geq 0$ & $\checkmark$ & $\checkmark$ & $\checkmark$ & $\checkmark$ & $\checkmark$ & $\checkmark$ & $\checkmark$ & $\checkmark$ & $\checkmark$ & $\checkmark$ & & & static \\
\hline $\mathrm{A} 22$ & $\geq 1$ & $\checkmark$ & & & $\checkmark$ & $\checkmark$ & $\checkmark$ & $\checkmark$ & $\checkmark$ & & & & $\neq 0$ & torsional static \\
\hline $\mathrm{A} 23_{\varepsilon}$ & $\geq 2$ & $\checkmark$ & $\checkmark$ & & $\checkmark$ & $\checkmark$ & $\checkmark$ & $\checkmark$ & $\checkmark$ & $\checkmark$ & $\checkmark$ & $\neq 0$ & & $\varepsilon= \pm 1$ \\
\hline $\mathrm{A} 24$ & 2 & $\checkmark$ & & & $\checkmark$ & $\checkmark$ & $\checkmark$ & $\checkmark$ & $\checkmark$ & & & & $\neq 0$ & \\
\hline
\end{tabular}

Table 16. Properties of simply-connected homogeneous spacetimes. This table describes if a spacetime of dimension $D+1$ is reductive $(\mathrm{R})$, symmetric $(\mathrm{S})$ or affine (A). A spacetime might exhibit a lorentzian $(\mathrm{L})$, riemannian $(\mathrm{E})$, galilean $(\mathrm{G})$ or carrollian $(\mathrm{C})$ structure, and be invariant under parity $(\mathrm{P})$, time reversal $(\mathrm{T})$ or their combination $(\mathrm{PT})$. Furthermore the canonical connection might be have curvature $(\Omega)$ and/or torsion $(\Theta)$. For the precise definitions of these properties see sections 6.1 and 6.2 .

\section{Conclusions}

In this paper we have classified isomorphism classes of simply-connected homogeneous spacetimes of kinematical and aristotelian Lie groups with $D$-dimensional space isotropy for all $D \geq 0$. We have done this by classifying the corresponding infinitesimal algebraic objects, namely (geometrically realisable, effective) Lie pairs. A number of observations follow from the classification.

It follows from our classification (see, e.g., table 15) that inequivalent spacetimes may have the same transitive kinematical Lie algebra, which might have interesting consequences (e.g., as in AdS/CFT). Conversely, non-isomorphic kinematical Lie algebras may have isomorphic spacetimes. For example, the para-galilean and static kinematical Lie algebras lead to the same homogeneous spacetime: the static aristotelian spacetime (A21). 
The classification yields novel (at least to us) spacetimes: particularly, the family of torsional galilean (anti) de Sitter spacetimes $\left(\mathrm{S} 9_{\gamma}\right.$ and $\left.\mathrm{S} 11_{\chi}\right)$ and the torsional static aristotelian spacetime (A22), as well as the new families of two- and three-dimensional spacetimes: $\mathrm{S} 12_{\gamma, \chi}, \mathrm{S} 19_{\chi}$ and $\mathrm{S} 20_{\chi}$. These novel spacetimes can be distinguished from the known ones (see figure 2) in one of several (equivalent) ways:

- they are not symmetric homogeneous spaces;

- they are not invariant under both parity and time-reversal (at least for $D \geq 2$ ); and

- they do not arise as limits of the maximally symmetric riemannian and lorentzian spaces.

In particular, this last characterisation allows us to see their existence as a purely nonrelativistic prediction. Conversely, one can ask whether there is a relativistic set-up that leads to these spacetimes via limits. None of these characterisations is compelling reason to ignore the novel spacetimes.

We observed that not all aristotelian spacetimes arise from kinematical Lie algebras and this motivated us to present the separate classification of aristotelian Lie algebras in appendix A.

We also explored some of the geometrical properties of the spacetimes, particularly those which can be easily read from the infinitesimal description: namely the existence of invariant (pseudo-)riemannian, galilean, carrollian and aristotelian structures. In the reductive cases, which are the vast majority, we have paid particular attention to the torsion and curvature of the canonical connection, as this provides an identifiable invariant for the spacetime in question.

The main results are contained in tables 1, 2 and 16 and their interrelations are conveniently summarised in figures 3,4 and 5. In this paper we have restricted ourselves to the classification of the simply-connected homogeneous spacetimes, without paying very close attention to each of the geometries. This will be remedied in a follow-up paper [54], where we will revisit the classification and investigate the local geometry of the spacetimes.

\section{Acknowledgments}

This work started during a visit of SP to Edinburgh and continued during a visit of JMF to Brussels. It is our pleasure to thank the corresponding institutions for hospitality and support. Additional work was done during our participation at the MITP Topical Workshop "Applied Newton-Cartan Geometry" (APPNC2018), held at the Mainz Institute for Theoretical Physics, where we presented a preliminary version of our results. We are grateful to the MITP for their support and hospitality and for providing such a stimulating research atmosphere. We are particularly grateful to Eric Bergshoeff and Niels Obers for the invitation to participate. JMF would like to thank Andrea Santi for the invitation to visit Bologna and the opportunity to talk about this work. SP is grateful to Eric Bergshoeff, Daniel Grumiller, Joaquim Gomis, Marc Henneaux, Axel Kleinschmidt, Victor Lekeu, Javier Matulich, Arash Ranjbar, Jan Rosseel, Jakob Salzer, Friedrich Schöller, and 
David Tong for useful discussions. The research of JMF is partially supported by the grant ST/L000458/1 "Particle Theory at the Higgs Centre" from the U.K. Science and Technology Facilities Council. The research of SP is partially supported by the ERC Advanced Grant "High-Spin-Grav" and by FNRS-Belgium (convention FRFC PDR T.1025.14 and convention IISN 4.4503.15).

JMF would like to dedicate this paper to the memory of his friend Andrew Ranicki, the late Professor of Algebraic Surgery at the University of Edinburgh. Andrew was a great practitioner in the art of turning topological/geometrical problems into algebra, which is the philosophy we have tried to follow in arriving at the results described in this paper.

\section{A Classification of aristotelian Lie algebras}

In this appendix we present the classification of aristotelian Lie algebras. In complete analogy with the definition of a kinematical Lie algebra, we have the following.

Definition 4. A real Lie algebra $\mathfrak{a}$ is said to be aristotelian (with $D$-dimensional space isotropy) if it satisfies two properties:

1. $\mathfrak{a}$ contains a Lie subalgebra $\mathfrak{r} \cong \mathfrak{s o}(D)$, and

2. $\mathfrak{a}$ decomposes as $\mathfrak{a}=\mathfrak{r} \oplus V \oplus S$ under $\mathfrak{r}$,

where now we only have one copy of the vector representation of $\mathfrak{s o}(D)$.

We can choose a basis $\left(J_{a b}, P_{a}, H\right)$ for $\mathfrak{a}$, relative to which the Lie brackets include the following:

$$
\begin{aligned}
{\left[J_{a b}, J_{c d}\right] } & =\delta_{b c} J_{a d}-\delta_{a c} J_{b d}-\delta_{b d} J_{a c}+\delta_{a d} J_{b c} \\
{\left[J_{a b}, P_{c}\right] } & =\delta_{b c} P_{a}-\delta_{a c} P_{b} \\
{\left[J_{a b}, H\right] } & =0
\end{aligned}
$$

and any other Lie brackets are subject only to the Jacobi identity, which implies, in particular, equivariance under $\mathfrak{r}$. Every aristotelian Lie algebra $\mathfrak{a}$ gives rise to a unique aristotelian homogeneous spacetime with effective Lie pair $(\mathfrak{a}, \mathfrak{r})$. Therefore classifying aristotelian Lie algebras up to isomorphism also classifies the aristotelian spacetimes. Aristotelian Lie algebras all share the Lie brackets (A.1) and are thus distinguished by the $\left[H, P_{a}\right]$ and $\left[P_{a}, P_{b}\right]$ brackets, which are only constrained by the Jacobi identity.

Many aristotelian Lie algebras arise as quotients of kinematical Lie algebras by a vectorial ideal. Indeed, we have seen in section 4 that kinematical Lie algebras giving rise to non-effective Lie pairs always reduce to an aristotelian Lie algebra after quotienting by the ideal generated by the boosts. However, as we shall see below, not all aristotelian Lie algebras arise in this way.

We now proceed to classify aristotelian Lie algebras, starting with those in $D>3$ and making our way down in dimension.

Let $D>3$. Equivariance under $\mathfrak{r} \cong \mathfrak{s o}(D)$ forces

$$
\left[H, P_{a}\right]=\alpha P_{a} \quad \text { and } \quad\left[P_{a}, P_{b}\right]=\beta J_{a b}
$$


for some $\alpha, \beta \in \mathbb{R}$. The Jacobi identity says that $\alpha \beta=0$, giving rise to four isomorphism classes of aristotelian Lie algebras:

(A1) the static aristotelian Lie algebra $(\alpha=\beta=0)$;

(A2) $\left[H, P_{a}\right]=P_{a}$ and $\left[P_{a}, P_{b}\right]=0(\alpha \neq 0, \beta=0)$; and

(A3) $\left[H, P_{a}\right]=0$ and $\left[P_{a}, P_{b}\right]=\varepsilon J_{a b}$, with $\varepsilon= \pm 1(\alpha=0, \beta \neq 0)$.

In $D=3$ equivariance under $\mathfrak{r} \cong \mathfrak{s o}(3)$ allows a further term

$$
\left[H, P_{a}\right]=\alpha P_{a} \quad \text { and } \quad\left[P_{a}, P_{b}\right]=\beta J_{a b}+\gamma \epsilon_{a b c} P_{c},
$$

for some $\alpha, \beta, \gamma \in \mathbb{R}$. The Jacobi identity now says that $\alpha \beta=\alpha \gamma=0$. But in $D=3$ we can change basis to $P_{a} \mapsto P_{a}^{\prime}=P_{a}+\lambda \epsilon_{a b c} J_{b c}$ for some $\lambda \in \mathbb{R}$, apart from an overall scale. Choosing $\lambda=\frac{1}{4} \gamma$, we can assume that $\gamma=0$ without loss of generality. In terms of the new basis, we are back to the case $D>3$ with the same results:

(A1) the static aristotelian Lie algebra $\left(\alpha=0, \beta=\frac{1}{4} \gamma^{2}\right)$;

(A2) $\left[H, P_{a}\right]=P_{a}$ and $\left[P_{a}, P_{b}\right]=0(\alpha \neq 0, \beta=\gamma=0)$; and

(A3) $\left[H, P_{a}\right]=0$ and $\left[P_{a}, P_{b}\right]=\varepsilon J_{a b}$, with $\varepsilon= \pm 1\left(\alpha=0, \beta \neq \frac{1}{4} \gamma^{2}\right)$.

It is only for $D=3$ that the aristotelian Lie algebra A3 arises by reduction from a kinematical Lie algebra. Indeed, since $J_{a b}$ can be written as a Lie bracket of translations and since boosts transform nontrivially under rotations, boosts cannot commute with translations in the kinematical Lie algebra. Since the boosts define an ideal, there would have to be a nonzero bracket $[\boldsymbol{B}, \boldsymbol{P}]=\boldsymbol{B}$, whose existence requires a nontrivial vector product invariant under rotations, and this only exists in $D=3$.

Let $D=2$. Now $\mathfrak{r} \cong \mathfrak{s o}(2)$ is abelian, so equivariance under $\mathfrak{r}$ implies

$$
\left[H, P_{a}\right]=\alpha P_{a}+\delta \epsilon_{a b} P_{b} \quad \text { and } \quad\left[P_{a}, P_{b}\right]=\epsilon_{a b}(\beta J+\gamma H),
$$

where we have defined $J$ via $J_{a b}=-\epsilon_{a b} J$. But now we can change basis to $H \mapsto H^{\prime}=$ $H-\lambda J$ for some $\lambda \in \mathbb{R}$, apart from an overall scale. Choosing $\lambda=\delta$, we can assume with no loss of generality that $\delta=0$. In general, the Jacobi identity says that $\alpha \beta=\alpha \gamma=0$. There is now an additional aristotelian Lie algebra:

(A1) the static aristotelian Lie algebra $(\alpha=\beta=\gamma=0)$;

(A2) $\left[H, P_{a}\right]=P_{a}$ and $\left[P_{a}, P_{b}\right]=0(\alpha \neq 0, \beta=\gamma=0)$;

(A3) $\left[H, P_{a}\right]=0$ and $\left[P_{a}, P_{b}\right]=\varepsilon J_{a b}$, with $\varepsilon= \pm 1(\alpha=0, \beta \neq \gamma \delta)$; and

(A4) $\left[P_{a}, P_{b}\right]=\epsilon_{a b} H(\alpha=0, \beta=\gamma \delta, \gamma \neq 0)$.

Here, because of the possibility of redefining $H$ and $J$, the aristotelian Lie algebra A3 can arise by reduction of a kinematical Lie algebra.

Let $D=1$. Here there are no rotations, so any two-dimensional Lie algebra is aristotelian. Up to isomorphism there are precisely two such Lie algebras: 


\begin{tabular}{|l|c|cc|l|}
\hline A\# & $D$ & Nonzero Lie brackets in addition to $[\boldsymbol{J}, \boldsymbol{J}]=\boldsymbol{J},[\boldsymbol{J}, \boldsymbol{P}]=\boldsymbol{P}$ & Comments \\
\hline 1 & $\geq 0$ & & static \\
2 & $\geq 1$ & {$\left[H, P_{a}\right]=P_{a}$} & \\
$3_{\varepsilon}$ & $\geq 2$ & {$\left[P_{a}, P_{b}\right]=\varepsilon J_{a b}$} & $\varepsilon= \pm 1$ \\
4 & 2 & {$\left[P_{a}, P_{b}\right]=\epsilon_{a b} H$} & \\
\hline
\end{tabular}

Table 17. Aristotelian Lie algebras.

(A1) the static aristotelian Lie algebra; and

(A2) $[H, P]=P$.

Finally, in $D=0$, there is only the one-dimensional Lie algebra spanned by $H$, which is the $D=0$ avatar of the static aristotelian Lie algebra A1.

In summary, the isomorphism classes of aristotelian Lie algebras with $D$-dimensional space isotropy are recorded in table 17.

\section{B Infinitesimal description of homogeneous spaces}

In this appendix we will prove that the classification of simply-connected homogeneous spaces (up to isomorphism) is equivalent to the classification of isomorphism classes of geometrically realisable, effective Lie pairs. This statement is the analogue in the homogeneous space setting of the well-known fact that associated to every finite-dimensional real Lie algebra $\mathfrak{g}$ there exists a unique (up to isomorphism) simply-connected Lie group whose Lie algebra is isomorphic to $\mathfrak{g}$. Indeed, a Lie group is a principally homogeneous space over itself, so that the group/algebra statement is a special case of the general statement about homogeneous spaces. The crucial difference is that given a Lie pair the corresponding simply-connected homogeneous space need not exist nor be unique, unless we impose additional conditions on the Lie pair: effective (for uniqueness) and geometrically realisable (for existence). It is surprisingly difficult to find this more general statement in the literature, but it is certainly standard and one can piece it together from results in [57]. For psychological reasons, in this appendix $\mathfrak{g}$ will denote a finite-dimensional real Lie algebra and not the galilean Lie algebra as in the bulk of the paper.

\section{B.1 Transitive actions of Lie groups}

Let $M$ be a connected smooth manifold. By an action of a Lie group $\mathcal{G}$ on $M$, we mean a smooth map $\alpha: \mathcal{G} \times M \rightarrow M$ satisfying axioms which are easier to state after we introduce the following notation. If $g \in \mathcal{G}$ and $m \in M$, we will write $\alpha(g, m)$ as $g \cdot m$. Then $\alpha$ is an action if, for $e \in \mathcal{G}$ the identity element, $e \cdot m=m$ for all $m \in M$, and if $g_{1} \cdot\left(g_{2} \cdot m\right)=\left(g_{1} g_{2}\right) \cdot m$ for all $g_{1}, g_{2} \in \mathcal{G}$ and $m \in M$.

Let $\mathcal{G}$ act on $M$ and let $\mathfrak{g}$ be the Lie algebra of $\mathcal{G}$. The action induces a Lie algebra (anti)homomorphism $\xi: \mathfrak{g} \rightarrow \mathscr{X}(M)$ from $\mathfrak{g}$ to the Lie algebra of vector fields on $M$ sending every $X \in \mathfrak{g}$ to the fundamental vector field $\xi_{X}$ on $M$. If $m \in M$, then $\xi_{X}(m)$ is the velocity of the curve $\exp (t X) \cdot m$ at $t=0$. 
Let $\mathcal{G}$ act on $M$ and let $\mathscr{N}=\{g \in \mathcal{G} \mid g \cdot m=m, \forall m \in M\}$ denote the kernel of the action. The action of $\mathcal{G}$ on $M$ is said to be effective if $\mathscr{N}=\{e\}$ and it is said to be locally effective if $\mathscr{N}$ is a discrete group. This is equivalent to the map $\xi: \mathfrak{g} \rightarrow \mathscr{X}(M)$ being injective. In any case, $\mathscr{N}$ is a normal subgroup of $\mathcal{G}$ and the $\mathcal{G}$ action on $M$ induces an effective action of $\mathcal{G} / \mathscr{N}$. Nevertheless, although assuming that the action is effective seems to represent no loss of generality, we will allow for locally effective actions.

Let $\mathcal{G}$ act on $M$. If given any two points $m_{1}, m_{2} \in M$, there is some $g \in \mathcal{G}$ such that $m_{2}=g \cdot m_{1}$, we say that the action is transitive. If the action is both transitive and locally effective, then $M$ is said to be a homogeneous space of $\mathcal{G}$. We will assume that $\mathcal{G}$ is connected. This represents no loss of generality because if $\mathcal{G}$ acts transitively on a connected manifold $M$, so does the connected component of the identity of $\mathcal{G}$.

Definition 5. Let $M$ and $M^{\prime}$ be homogeneous spaces of $\mathcal{G}$ and $\mathcal{G}^{\prime}$, respectively. We say that $M$ and $M^{\prime}$ are isomorphic if there is a diffeomorphism $f: M \rightarrow M^{\prime}$ and a Lie group isomorphism $\Phi: \mathcal{G} \rightarrow \mathcal{G}^{\prime}$ such that $f(g \cdot m)=\Phi(g) \cdot f(m)$ for all $m \in M$ and $g \in \mathcal{G}$.

Let $\mathcal{G}$ be a connected Lie group acting transitively on $M$ and let $m \in M$. The set $\mathcal{G}_{m}=\{g \in \mathcal{G} \mid g \cdot m=m\}$ is a closed Lie subgroup of $\mathcal{G}$ called the stabiliser of $m$. It need not be a connected subgroup. Pick an "origin" $o \in M$ and let $\mathcal{H}=\mathcal{G}_{o}$. Then $M$ is diffeomorphic to the space $\mathcal{G} / \mathcal{H}$ of right $\mathcal{H}$-cosets. The diffeomorphism $\iota: M \rightarrow \mathcal{G} / \mathcal{H}$ is such that $\iota(o)=e \mathcal{H}$ and if $m=g \cdot o$ then $\iota(m)=g \mathcal{H}$, which is well-defined because $\mathcal{H}$ is the stabiliser of $o$. It follows that $\iota$ is $\mathcal{G}$-equivariant: $\iota(g \cdot m)=g \iota(m)$ for all $g \in \mathcal{G}$ and $m \in M$. In the language of the previous definition, the homogeneous spaces $M$ and $\mathcal{G} / \mathcal{H}$ of $\mathcal{G}$ are isomorphic. We say that $\mathcal{G} / \mathcal{H}$ is a coset model for $M$.

If we change the origin, we get a different (but isomorphic) coset model. Indeed, let $o^{\prime} \in M$ have stabiliser $\mathcal{H}^{\prime}$. Then if $o^{\prime}=g \cdot o, \mathcal{H}^{\prime}=g \mathcal{H} g^{-1}$ and in the language of the above definition, $\Phi: \mathcal{G} \rightarrow \mathcal{G}$ is the inner automorphism corresponding to conjugation by $g$ and $f: \mathcal{G} / \mathcal{H} \rightarrow \mathcal{G} / \mathcal{H}^{\prime}$ is such that $f(k \mathcal{H})=g k g^{-1} \mathcal{H}^{\prime}$.

\section{B.2 Lie pairs}

Every coset space $\mathcal{G} / \mathcal{H}$ has a corresponding Lie pair $(\mathfrak{g}, \mathfrak{h})$, where $\mathfrak{g}$ is the Lie algebra of $\mathcal{G}$ and $\mathfrak{h}$ is the Lie algebra of $\mathcal{H}$, and so there is a way to assign a Lie pair to a homogeneous space $M$ of $\mathcal{G}$ and a choice of origin. A different choice of origin results in a different Lie pair, but how are they related? Let $o, o^{\prime} \in M$ be two choices of origin with stabilisers $\mathcal{H}$ and $\mathcal{H}^{\prime}=g \mathcal{H} g^{-1}$, where $g \cdot o=o^{\prime}$. Then the resulting Lie pairs are $(\mathfrak{g}, \mathfrak{h})$ and $\left(\mathfrak{g}, \mathfrak{h}^{\prime}\right)$, where $\mathfrak{h}^{\prime}$ is the Lie algebra of $\mathcal{H}^{\prime}$. Let $\operatorname{Ad}_{g}: \mathfrak{g} \rightarrow \mathfrak{g}$ be the inner automorphism of $\mathfrak{g}$ induced by conjugation by $g$ in $\mathcal{G}$. Then $\mathfrak{h}^{\prime}=\operatorname{Ad}_{g} \mathfrak{h}$, so the Lie pairs are related by an inner automorphism. This motivates the following definition.

Definition 6. Two Lie pairs $\left(\mathfrak{g}_{1}, \mathfrak{h}_{1}\right)$ and $\left(\mathfrak{g}_{2}, \mathfrak{h}_{2}\right)$ are said to be isomorphic if there is a Lie algebra isomorphism $\varphi: \mathfrak{g}_{1} \rightarrow \mathfrak{g}_{2}$ with $\varphi\left(\mathfrak{h}_{1}\right)=\mathfrak{h}_{2}$.

This notion of isomorphism is stronger than what is needed in order to classify homogeneous spaces, but it is easier to implement algebraically. In this appendix we will show 
that it corresponds to classifying homogeneous spaces up to coverings. Equivalently, we will see that to each isomorphism class of (certain) Lie pairs there corresponds a unique simply connected homogeneous space (up to isomorphism).

Lemma 1. Let $M$ and $M^{\prime}$ be homogeneous spaces of $\mathcal{G}$ and $\mathcal{G}^{\prime}$, respectively. If $M$ and $M^{\prime}$ are isomorphic, then so are any Lie pairs associated to $M$ and $M^{\prime}$.

Proof. It is enough to show that any Lie pair associated to $M$ is isomorphic to at least one Lie pair associated to $M^{\prime}$, since as we have seen above all Lie pairs associated to a homogeneous space are isomorphic (by an inner automorphism). Since $M$ and $M^{\prime}$ are isomorphic homogeneous spaces, we have an isomorphism of Lie groups $\Phi: \mathcal{G} \rightarrow \mathcal{G}^{\prime}$ and a diffeomorphism $f: M \rightarrow M^{\prime}$ obeying the equivariance property $f(g \cdot m)=\Phi(g) \cdot f(m)$ for all $m \in M$ and $g \in \mathcal{G}$. We will show that the Lie algebra isomorphism $\varphi: \mathfrak{g} \rightarrow \mathfrak{g}^{\prime}$ induced by $\Phi$ is the desired isomorphism between the Lie pairs.

So choose an origin $o \in M$ with stabiliser $\mathcal{H} \subset \mathcal{G}$, leading to the Lie pair $(\mathfrak{g}, \mathfrak{h})$ and let $o^{\prime}=f(o) \in M^{\prime}$ have stabiliser $\mathcal{H}^{\prime}$, leading to the Lie pair $\left(\mathfrak{g}^{\prime}, \mathfrak{h}^{\prime}\right)$. It follows from the equivariance property that if $g \in \mathcal{H}$, then $\Phi(g) \in \mathcal{H}^{\prime}$ :

$$
\Phi(g) \cdot o^{\prime}=\Phi(g) \cdot f(o)=f(g \cdot o)=f(o)=o^{\prime} .
$$

But if $g^{\prime} \in \mathcal{H}^{\prime}$, then the unique $g \in \mathcal{G}$ such that $g^{\prime}=\Phi(g)$ lies in $\mathcal{H}$ :

$$
f(o)=o^{\prime}=g^{\prime} \cdot o^{\prime}=\Phi(g) \cdot f(o)=f(g \cdot o),
$$

but since $f$ is one-to-one, $g \cdot o=o$. Therefore $\Phi(\mathcal{H})=\mathcal{H}^{\prime}$ and the Lie algebra isomorphism $\varphi: \mathfrak{g} \rightarrow \mathfrak{g}^{\prime}$ induced by $\Phi$ sends $\mathfrak{h}$ isomorphically to $\mathfrak{h}^{\prime}$.

It turns out that not all Lie pairs come from homogeneous spaces.

Definition 7. A Lie pair $(\mathfrak{g}, \mathfrak{h})$ is said to be effective if $\mathfrak{h}$ does not contain a nonzero ideal of $\mathfrak{g}$.

It follows from this definition that if two Lie pairs are isomorphic and one is effective, so is the other. The following lemma justifies the definition.

Lemma 2. Let $M=\mathcal{G} / \mathcal{H}$ be a coset space with Lie pair $(\mathfrak{g}, \mathfrak{h})$. Then $(\mathfrak{g}, \mathfrak{h})$ is effective if and only if the action of $\mathcal{G}$ on $M$ is locally effective.

Proof. We start by proving that if $(\mathfrak{g}, \mathfrak{h})$ is not effective, then $\mathcal{G}$ does not act locally effectively. If $(\mathfrak{g}, \mathfrak{h})$ is not effective, then there is a nonzero ideal $\mathfrak{n}$ of $\mathfrak{g}$ contained in $\mathfrak{h}$. Let $\mathscr{N}$ be the unique connected subgroup of $\mathcal{G}$ generated by $\mathfrak{n}$. Since $\mathfrak{n}$ is an ideal, $\mathscr{N}$ is a normal subgroup. We claim that $\mathscr{N}$ stabilises every point on $M$. Since $\mathscr{N} \subset \mathcal{H}$, it stabilises any point $o \in M$ with stabiliser $\mathcal{H}$. Let $m \in M$ be any other point and let $g \in \mathcal{G}$ be such that $g \cdot o=m$. Then the stabiliser of $m$ is $g \mathcal{H} g^{-1}$, which contains $g \mathscr{N} g^{-1}=\mathscr{N}$.

Conversely, suppose that $\mathcal{G}$ does not act locally effectively, so that the Lie algebra (anti)homomorphism $\xi: \mathfrak{g} \rightarrow \mathscr{X}(M)$ has nonzero kernel $\mathfrak{n}$, which is an ideal of $\mathfrak{g}$. Let $o \in M$ have stabiliser $\mathcal{H}$. Then $\mathfrak{h}$ consists of those $X \in \mathfrak{g}$ for which $\xi_{X}(o)=0$. But if $X \in \mathfrak{n}, \xi_{X}(m)=0$ for all $m \in M$, so in particular, $\xi_{X}(o)=0$ and hence $X \in \mathfrak{h}$. This means that there is an ideal of $\mathfrak{g}$ contained in $\mathfrak{h}$ and hence $(\mathfrak{g}, \mathfrak{h})$ is not effective. 
In summary, to a homogeneous space of $\mathcal{G}$ and a choice of origin, we may assign an effective Lie pair and up to isomorphism the choice of origin is immaterial. We now wish to examine the inverse problem: namely, does every effective Lie pair arise as the Lie pair of a homogeneous space and a choice of origin?

\section{B.3 Geometric realisations}

It turns out that not every effective Lie pair arises from a homogeneous space. For example, consider $\mathfrak{g}=\mathfrak{s u}(3)$, the simple Lie algebra of $3 \times 3$ traceless skewhermitian complex matrices, and let $\mathfrak{h}$ be the one-dimensional subalgebra spanned by the matrix

$$
X_{\alpha}=\left(\begin{array}{ccc}
i & 0 & 0 \\
0 & \alpha i & 0 \\
0 & 0 & -(1+\alpha) i
\end{array}\right)
$$

for some irrational real number $\alpha$. We claim that there is no Lie group $\mathcal{G}$ with Lie algebra isomorphic to $\mathfrak{g}$ for which the subgroup corresponding to $\mathfrak{h}$ is closed. Indeed, there are (up to isomorphism) precisely two connected Lie groups with Lie algebra isomorphic to $\mathfrak{s u}(3)$ : $\mathrm{SU}(3)$ itself and the adjoint group $\mathrm{Ad} \mathrm{SU}(3) \cong \mathrm{SU}(3) / \mathbb{Z}_{3}$. The one-parameter subgroup of either of these groups generated by $X_{\alpha}$ is not closed. It is enough to see this for the simplyconnected group $\mathrm{SU}(3)$, since if the subgroup of $\mathrm{SU}(3) / \mathbb{Z}_{3}$ generated by $X_{\alpha}$ were closed, then so would be its pre-image under the covering homomorphism $\pi: \mathrm{SU}(3) \rightarrow \mathrm{SU}(3) / \mathbb{Z}_{3}$, which is the subgroup generated by $X_{\alpha}$ in $\mathrm{SU}(3)$. So let $H_{\alpha}$ denote the subgroup generated by $X_{\alpha}$ in $\mathrm{SU}(3)$. It is clearly contained in the maximal torus of diagonal matrices in $\mathrm{SU}(3)$, which is a closed subgroup. So it defines a one-parameter subgroup of the torus with an irrational slope and it's easy to see that the closure of this subgroup is the whole torus. ${ }^{4}$

This suggests the following definition.

Definition 8. A Lie pair $(\mathfrak{g}, \mathfrak{h})$ is geometrically realisable if there is a connected Lie group $\mathcal{G}$ with Lie algebra $\operatorname{Lie}(\mathcal{G})$ isomorphic to $\mathfrak{g}$ and a closed Lie subgroup $\mathcal{H}$ with Lie algebra $\operatorname{Lie}(\mathcal{H})$ isomorphic to $\mathfrak{h}$ (by restricting the isomorphism $\operatorname{Lie}(\mathcal{G}) \cong \mathfrak{g}$ ). The coset space $\mathcal{G} / \mathcal{H}$ is then a geometric realisation of $(\mathfrak{g}, \mathfrak{h})$.

It is clear from this definition that if two Lie pairs are isomorphic and one pair admits a geometric realisation then so does the other pair.

\section{B.4 Simply-connected homogeneous spaces}

Finally, we are ready to prove the main result of this appendix. Namely, we show that every geometrically realisable, effective Lie pair admits a unique (up to isomorphism) simplyconnected geometric realisation.

Let $M:=\mathcal{G} / \mathcal{H}$ be a geometric realisation of the Lie pair $(\mathfrak{g}, \mathfrak{h})$, where $\mathcal{G}$ is connected. Let $\pi: \widetilde{\mathcal{G}} \rightarrow \mathcal{G}$ be the universal covering group of $\mathcal{G}$. Since $\pi$ is surjective, $\widetilde{\mathcal{G}}$ also acts

\footnotetext{
${ }^{4}$ Since the counterexample here is the irrational slope flow on a torus, one might have wondered why we didn't simply consider the abelian Lie algebra $\mathfrak{g}=\mathfrak{u}(1) \oplus \mathfrak{u}(1)$ and the subalgebra $\mathfrak{h}$ spanned by $(i, \alpha i)$, with $\alpha$ irrational. Indeed, the subgroup of $\mathrm{U}(1) \times \mathrm{U}(1)$ generated by $\mathfrak{h}$ is not closed, but the subgroup generated by $\mathfrak{h}$ in the universal covering group $\mathbb{R}^{2}$ is closed, so that the Lie pair $(\mathfrak{g}, \mathfrak{h})$ is geometrically realisable.
} 
transitively on $M$ via $g \cdot m=\pi(g) \cdot m$, for $g \in \widetilde{\mathcal{G}}$ and $m \in M$. If $o \in M$ denotes the identity coset, then its stabiliser in $\widetilde{\mathcal{G}}$ is $\widetilde{\mathcal{H}}=\pi^{-1} \mathcal{H}=\{h \in \widetilde{\mathcal{G}} \mid \pi(g) \in \mathcal{H}\}$. Therefore $M=\widetilde{\mathcal{G}} / \widetilde{\mathcal{H}}=\mathcal{G} / \mathcal{H}$. Now let $\widetilde{\mathcal{H}}_{1}$ denote the connected component of the identity in $\widetilde{\mathcal{H}}$ and let $\widetilde{M}:=\widetilde{\mathcal{G}} / \widetilde{\mathcal{H}}_{1}$.

Lemma 3. $\widetilde{M}$ is the universal cover of $M$ and the covering map $p: \widetilde{M} \rightarrow M$ is $\widetilde{\mathcal{G}}$ equivariant. Furthermore the Lie pair associated to $\widetilde{M}$ is isomorphic to $(\mathfrak{g}, \mathfrak{h})$.

Proof. It is clear that $\widetilde{M}$ is a homogeneous space of $\widetilde{\mathcal{G}}$ and hence it is the base of a principal $\widetilde{\mathcal{H}}_{1}$-bundle

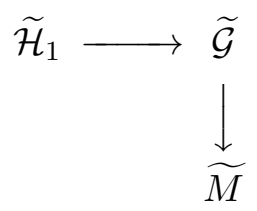

whose homotopy long exact sequence ends with

$$
\pi_{1}(\widetilde{\mathcal{G}}) \longrightarrow \pi_{1}(\widetilde{M}) \longrightarrow \pi_{0}\left(\widetilde{\mathcal{H}}_{1}\right) \longrightarrow \pi_{0}(\widetilde{\mathcal{G}}) \longrightarrow 0
$$

where all maps are group homomorphisms. Since $\widetilde{\mathcal{G}}$ is connected and simply connected, $\pi_{0}(\widetilde{\mathcal{G}})=\pi_{1}(\widetilde{\mathcal{G}})=0$ and since $\widetilde{\mathcal{H}}_{1}$ is connected, $\pi_{0}\left(\widetilde{\mathcal{H}}_{1}\right)=0$, resulting in $\pi_{1}(\widetilde{M})=0$. The map $p: \widetilde{M}=\widetilde{\mathcal{G}} / \widetilde{\mathcal{H}}_{1} \rightarrow M=\widetilde{\mathcal{G}} / \widetilde{\mathcal{H}}$, defined by $p\left(g \widetilde{\mathcal{H}}_{1}\right)=g \widetilde{\mathcal{H}}$, is manifestly $\widetilde{\mathcal{G}}$-equivariant and moreover it is a covering since it is the projection of a principal bundle with base $M$ and discrete fibre $\pi_{0}(\widetilde{\mathcal{H}})$. The Lie pair associated to $\widetilde{M}$ is $\left(\operatorname{Lie}(\widetilde{\mathcal{G}}), \operatorname{Lie}\left(\widetilde{\mathcal{H}}_{1}\right)\right)$, but since $\pi: \widetilde{\mathcal{G}} \rightarrow \mathcal{G}$ is a covering homomorphism of Lie groups, the Lie map $\pi_{*}: \operatorname{Lie}(\widetilde{\mathcal{G}}) \rightarrow \mathfrak{g}$ is a Lie algebra isomorphism which restricts to an isomorphism $\operatorname{Lie}\left(\widetilde{\mathcal{H}}_{1}\right)=\operatorname{Lie}(\widetilde{\mathcal{H}}) \rightarrow \mathfrak{h}$. Hence the Lie pairs $\left(\operatorname{Lie}(\widetilde{\mathcal{G}}), \operatorname{Lie}\left(\widetilde{\mathcal{H}}_{1}\right)\right)$ and $(\mathfrak{g}, \mathfrak{h})$ are isomorphic.

We have shown that every geometrically realisable Lie pair $(\mathfrak{g}, \mathfrak{h})$ has a simplyconnected geometric realisation $\widetilde{M}$ as above. It turns out that this is unique up to isomorphism.

Lemma 4. $\widetilde{M}$ is the unique (up to isomorphism) simply-connected geometric realisation of $(\mathfrak{g}, \mathfrak{h})$.

Proof. Suppose that $\widetilde{M}^{\prime}$ is another simply-connected geometric realisation of $(\mathfrak{g}, \mathfrak{h})$. This means that there is a connected Lie group $\mathcal{G}^{\prime}$ and a closed subgroup $\mathcal{H}^{\prime}$ such that $\widetilde{M^{\prime}}=\mathcal{G}^{\prime} / \mathcal{H}^{\prime}$ and such that the Lie pairs $\left(\mathfrak{g}^{\prime}, \mathfrak{h}^{\prime}\right)$ and $(\mathfrak{g}, \mathfrak{h})$ are isomorphic, where $\mathfrak{g}^{\prime}$ and $\mathfrak{h}^{\prime}$ are the Lie algebras of $\mathcal{G}^{\prime}$ and $\mathcal{H}^{\prime}$, respectively. This also means that $\left(\mathfrak{g}^{\prime}, \mathfrak{h}^{\prime}\right)$ is isomorphic to the Lie pair $\left(\operatorname{Lie}(\widetilde{\mathcal{G}}), \operatorname{Lie}\left(\widetilde{\mathcal{H}}_{1}\right)\right)$ of $\widetilde{M}$. Let $\varphi:\left(\mathfrak{g}^{\prime}, \mathfrak{h}^{\prime}\right) \rightarrow\left(\operatorname{Lie}(\widetilde{\mathcal{G}}), \operatorname{Lie}\left(\widetilde{\mathcal{H}}_{1}\right)\right)$ denote this isomorphism. Passing to the universal covering group (if necessary), we may assume without loss of generality that $\mathcal{G}^{\prime}$ is simply-connected and, since $\widetilde{M^{\prime}}=\mathcal{G}^{\prime} / \mathcal{H}^{\prime}$ is simply connected, that $\mathcal{H}^{\prime}$ is connected. The isomorphism $\varphi: \mathfrak{g}^{\prime} \rightarrow \operatorname{Lie}(\widetilde{\mathcal{G}})$ lifts to a unique Lie group isomorphism $\Phi: \mathcal{G}^{\prime} \rightarrow \widetilde{\mathcal{G}}$ which restricts to an isomorphism $\mathcal{H}^{\prime} \rightarrow \widetilde{\mathcal{H}}_{1}$ and hence induces a unique isomorphism of homogeneous spaces $\phi: \widetilde{M}^{\prime}=\mathcal{G}^{\prime} / \mathcal{H}^{\prime} \rightarrow \widetilde{M}=\widetilde{\mathcal{G}} / \widetilde{\mathcal{H}}_{1}$, sending $g \mathcal{H}^{\prime} \mapsto \Phi(g) \widetilde{\mathcal{H}}_{1}$. 
As a corollary of the above lemma, we see that two homogeneous spaces with isomorphic Lie pairs have isomorphic universal covers. Indeed, let $M=\mathcal{G} / \mathcal{H}$ and $M^{\prime}=\mathcal{G}^{\prime} / \mathcal{H}^{\prime}$ have isomorphic Lie pairs $(\mathfrak{g}, \mathfrak{h})$ and $\left(\mathfrak{g}^{\prime}, \mathfrak{h}^{\prime}\right)$, respectively. Then the universal cover $\widetilde{M}$ of $M$ has a Lie pair which is isomorphic to $(\mathfrak{g}, \mathfrak{h})$ and the universal cover $\widetilde{M^{\prime}}$ of $M^{\prime}$ has a Lie pair which is isomorphic to $\left(\mathfrak{g}^{\prime}, \mathfrak{h}^{\prime}\right)$ and hence also to $(\mathfrak{g}, \mathfrak{h})$. Therefore $\widetilde{M}$ and $\widetilde{M}^{\prime}$ are simply-connected geometric realisations of $(\mathfrak{g}, \mathfrak{h})$ and by Lemma 4 they are isomorphic as homogeneous spaces.

In summary, we have proved the following.

Theorem 5. Isomorphism classes of geometrically realisable, effective Lie pairs are in oneto-one correspondence with isomorphism classes of simply-connected homogeneous spaces.

We may paraphrase this result as follows. Introduce an equivalence relation between homogeneous spaces by declaring two homogeneous spaces to be equivalent if their universal covers are isomorphic as homogeneous spaces. The isomorphism classes of geometrically realisable, effective Lie pairs are in one-to-one correspondence with equivalence classes of homogeneous spaces.

If we wish to classify homogeneous spaces up to isomorphism and not just up to covering, we would start from the classification of simply-connected homogeneous spaces and then classify their homogeneous quotients. That, however, is beyond the scope of this paper.

Open Access. This article is distributed under the terms of the Creative Commons Attribution License (CC-BY 4.0), which permits any use, distribution and reproduction in any medium, provided the original author(s) and source are credited.

\section{References}

[1] H. Bacry and J. Levy-Leblond, Possible kinematics, J. Math. Phys. 9 (1968) 1605 [INSPIRE].

[2] H. Bacry and J. Nuyts, Classification of ten-dimensional kinematical groups with space isotropy, J. Math. Phys. 27 (1986) 2455.

[3] J.M. Figueroa-O'Farrill, Kinematical Lie algebras via deformation theory, J. Math. Phys. 59 (2018) 061701 [arXiv:1711.06111] [INSPIRE].

[4] J.M. Figueroa-O'Farrill, Higher-dimensional kinematical Lie algebras via deformation theory, J. Math. Phys. 59 (2018) 061702 [arXiv:1711.07363] [INSPIRE].

[5] T. Andrzejewski and J.M. Figueroa-O'Farrill, Kinematical Lie algebras in $2+1$ dimensions, J. Math. Phys. 59 (2018) 061703 [arXiv: 1802.04048] [INSPIRE].

[6] J.M. Maldacena, The Large $N$ limit of superconformal field theories and supergravity, Int. J. Theor. Phys. 38 (1999) 1113 [hep-th/9711200] [INSPIRE].

[7] D.T. Son, Toward an AdS/cold atoms correspondence: A Geometric realization of the Schrödinger symmetry, Phys. Rev. D 78 (2008) 046003 [arXiv: 0804.3972] [InSPIRE].

[8] K. Balasubramanian and J. McGreevy, Gravity duals for non-relativistic CFTs, Phys. Rev. Lett. 101 (2008) 061601 [arXiv:0804.4053] [INSPIRE]. 
[9] S. Kachru, X. Liu and M. Mulligan, Gravity duals of Lifshitz-like fixed points, Phys. Rev. D 78 (2008) 106005 [arXiv: 0808.1725] [INSPIRE].

[10] M.H. Christensen, J. Hartong, N.A. Obers and B. Rollier, Torsional Newton-Cartan geometry and Lifshitz holography, Phys. Rev. D 89 (2014) 061901 [arXiv:1311.4794] [INSPIRE].

[11] M.H. Christensen, J. Hartong, N.A. Obers and B. Rollier, Boundary stress-energy tensor and Newton-Cartan geometry in Lifshitz holography, JHEP 01 (2014) 057 [arXiv:1311.6471] [INSPIRE].

[12] M. Taylor, Lifshitz holography, Class. Quant. Grav. 33 (2016) 033001 [arXiv:1512.03554] [INSPIRE].

[13] S.A. Hartnoll, A. Lucas and S. Sachdev, Holographic quantum matter, arXiv:1612.07324 [INSPIRE].

[14] S. Schäfer-Nameki, M. Yamazaki and K. Yoshida, Coset construction for duals of non-relativistic CFTs, JHEP 05 (2009) 038 [arXiv:0903.4245] [INSPIRE].

[15] J.I. Jottar, R.G. Leigh, D. Minic and L.A. Pando Zayas, Aging and holography, JHEP 11 (2010) 034 [arXiv: 1004.3752] [INSPIRE].

[16] A. Bagchi and A. Kundu, Metrics with galilean conformal isometry, Phys. Rev. D 83 (2011) 066018 [arXiv: 1011.4999] [INSPIRE].

[17] C. Duval and S. Lazzarini, Schrödinger manifolds, J. Phys. A 45 (2012) 395203 [arXiv: 1201.0683] [INSPIRE].

[18] K.T. Grosvenor, J. Hartong, C. Keeler and N.A. Obers, Homogeneous nonrelativistic geometries as coset spaces, Class. Quant. Grav. 35 (2018) 175007 [arXiv:1712.03980] [INSPIRE].

[19] D.T. Son, Newton-Cartan geometry and the quantum Hall effect, arXiv:1306.0638 [INSPIRE].

[20] M. Geracie, D.T. Son, C. Wu and S.-F. Wu, Spacetime symmetries of the quantum Hall effect, Phys. Rev. D 91 (2015) 045030 [arXiv:1407.1252] [INSPIRE].

[21] K. Jensen, On the coupling of Galilean-invariant field theories to curved spacetime, SciPost Phys. 5 (2018) 011 [arXiv: 1408.6855] [INSPIRE].

[22] T. Brauner, S. Endlich, A. Monin and R. Penco, General coordinate invariance in quantum many-body systems, Phys. Rev. D 90 (2014) 105016 [arXiv:1407.7730] [INSPIRE].

[23] R. Aldrovandi, A.L. Barbosa, L.C.B. Crispino and J.G. Pereira, Non-relativistic spacetimes with cosmological constant, Class. Quant. Grav. 16 (1999) 495 [gr-qc/9801100] [INSPIRE].

[24] M. Henneaux, Geometry of zero signature space-times, Bull. Soc. Math. Belg. 31 (1979) 47 [INSPIRE].

[25] C. Bunster and M. Henneaux, Duality invariance implies Poincaré invariance, Phys. Rev. Lett. 110 (2013) 011603 [arXiv: 1208.6302] [INSPIRE].

[26] C. Duval, G.W. Gibbons and P.A. Horvathy, Conformal Carroll groups and BMS symmetry, Class. Quant. Grav. 31 (2014) 092001 [arXiv:1402.5894] [INSPIRE].

[27] R. Andringa, E. Bergshoeff, S. Panda and M. de Roo, Newtonian gravity and the Bargmann algebra, Class. Quant. Grav. 28 (2011) 105011 [arXiv:1011.1145] [INSPIRE]. 
[28] J. Hartong, Gauging the Carroll algebra and ultra-relativistic gravity, JHEP 08 (2015) 069 [arXiv: 1505.05011] [INSPIRE].

[29] E. Bergshoeff et al., Carroll versus Galilei gravity, JHEP 03 (2017) 165 [arXiv:1701.06156] [INSPIRE].

[30] X. Bekaert and K. Morand, Connections and dynamical trajectories in generalised Newton-Cartan gravity I. An intrinsic view, J. Math. Phys. 57 (2016) 022507 [arXiv: 1412.8212] [INSPIRE].

[31] X. Bekaert and K. Morand, Connections and dynamical trajectories in generalised Newton-Cartan gravity II. An ambient perspective, J. Math. Phys. 59 (2018) 072503 [arXiv: 1505.03739] [INSPIRE].

[32] G. Papageorgiou and B.J. Schroers, A Chern-Simons approach to Galilean quantum gravity in $2+1$ dimensions, JHEP 11 (2009) 009 [arXiv:0907.2880] [INSPIRE].

[33] G. Papageorgiou and B.J. Schroers, Galilean quantum gravity with cosmological constant and the extended q-Heisenberg algebra, JHEP 11 (2010) 020 [arXiv:1008.0279] [INSPIRE].

[34] E.A. Bergshoeff and J. Rosseel, Three-dimensional extended bargmann supergravity, Phys. Rev. Lett. 116 (2016) 251601 [arXiv: 1604.08042] [INSPIRE].

[35] J. Hartong, Y. Lei and N.A. Obers, Nonrelativistic Chern-Simons theories and three-dimensional Hoŕava-Lifshitz gravity, Phys. Rev. D 94 (2016) 065027 [arXiv: 1604.08054] [INSPIRE].

[36] E. Bergshoeff, D. Grumiller, S. Prohazka and J. Rosseel, Three-dimensional spin-3 theories based on general kinematical algebras, JHEP 01 (2017) 114 [arXiv:1612.02277] [INSPIRE].

[37] E. Joung and W. Li, Nonrelativistic limits of colored gravity in three dimensions, Phys. Rev. D 97 (2018) 105020 [arXiv: 1801.10143] [INSPIRE].

[38] M. Le Bellac and J.M. Lévy-Leblond, Galilean electromagnetism, Nuovo Cim. B 14 (1973) 217.

[39] C. Duval, G.W. Gibbons, P.A. Horvathy and P.M. Zhang, Carroll versus Newton and Galilei: two dual non-Einsteinian concepts of time, Class. Quant. Grav. 31 (2014) 085016 [arXiv: 1402.0657] [INSPIRE].

[40] A. Bagchi, R. Basu and A. Mehra, Galilean conformal electrodynamics, JHEP 11 (2014) 061 [arXiv: 1408.0810] [INSPIRE].

[41] G. Festuccia, D. Hansen, J. Hartong and N.A. Obers, Symmetries and couplings of non-relativistic electrodynamics, JHEP 11 (2016) 037 [arXiv:1607.01753] [INSPIRE].

[42] R. Basu and U.N. Chowdhury, Dynamical structure of Carrollian electrodynamics, JHEP 04 (2018) 111 [arXiv: 1802.09366] [INSPIRE].

[43] D. Hansen, J. Hartong and N.A. Obers, An action principle for newtonian gravity, arXiv: 1807.04765 [INSPIRE].

[44] J.M. Lévy-Leblond, Une nouvelle limite non-relativiste du groupe de Poincaré, Ann. Inst. H. Poincaré A 3 (1965) 1.

[45] J.R. Derome and J.G. Dubois, Hooke's symmetries and nonrelativistic cosmological kinematics - I, Nuovo Cim. B 9 (1972) 351.

[46] J.F. Carinena, M.A. Del Olmo and M. Santander, Kinematic groups and dimensional analysis, J. Phys. A 14 (1981) 1 [inSPIRE]. 
[47] G.W. Gibbons and C.E. Patricot, Newton-Hooke space-times, Hpp waves and the cosmological constant, Class. Quant. Grav. 20 (2003) 5225 [hep-th/0308200] [INSPIRE].

[48] L. Bianchi, Sugli spazi a tre dimensioni che ammettono un gruppo continuo di movimenti, Mem. Mat. Fis. Soc. It. Sci. Ser III 11 (1898) 267.

[49] L. Bianchi, On the three-dimensional spaces which admit a continuous group of motions, Gen. Rel. Grav. 33 (2001) 2171, translated from the Italian by R. Jantzen.

[50] J.M. Figueroa-O'Farrill, Deformations of the Galilean algebra, J. Math. Phys. 30 (1989) 2735.

[51] N. Jacobson, Lie algebras, reprint of the 1962 original, Dover Publications, Inc., New York U.S.A. (1979).

[52] W. Rossmann, Lie groups, Oxford Graduate Texts in Mathematics volume 5, Oxford University Press, Oxford U.K. (2002).

[53] E. Weimar-Woods, The three-dimensional real Lie algebras and their contractions, J. Math. Phys. 32 (1991) 2028.

[54] J. M. Figueroa-O'Farrill, R. Grassie, and S. Prohazka, Geometry of spatially isotropic homogeneous spacetimes, in preparation.

[55] K. Nomizu, Invariant affine connections on homogeneous spaces, Amer. J. Math. 76 (1954) 33.

[56] S. Kobayashi and K. Nomizu, Foundations of differential geometry. Vol. II, reprint of the 1969 original, Wiley Classics Library. John Wiley \& Sons, Inc., New York U.S.A. (1996).

[57] V.V. Gorbatsevich, A.L. Onishchik and E.B. Vinberg, Foundations of Lie theory and Lie transformation groups, Springer, Berlin Germany (1997), translated from the Russian by A. Kozlowski. 